\title{
MODIFICATIONS OF HODGE BUNDLES AND ENUMERATIVE GEOMETRY : THE STABLE HYPERELLIPTIC LOCUS
}

\author{
ZIV RAN
}

\begin{abstract}
We study the stable hyperelliptic locus, i.e. the closure, in the Deligne- Mumford Moduli of stable curves, of the locus of smooth hyperelliptic curves. Working on a suitable blowup of the relative Hilbert scheme (of degree 2) associated to a family of stable curves, we construct a bundle map ('degree-2 Brill-Noether') from a modification of the Hodge bundle to a tautological bundle, whose degeneracy locus is the natural lift of the stable hyperelliptic locus plus a simple residual scheme. Using intersection theory on Hilbert schemes and Fulton-MacPherson residual intersection theory, the class of the structure sheaf and various other sheaves supported on the stable hyperelliptic locus can be computed by the Porteous formula and similar tools.
\end{abstract}

\section{CONTENTS}

Introduction

Further developments

Acknowledgment

\section{Part 1. Semicompact type}

0.1. Riemann-Roch without denominators for anti-self-dual 5

1. Locus of smooth hyperelliptics: a review $\quad 7$

1.1. Normal bundle

1.2. Normal forms

1.3. Pointed case

2. Good families and their Hilbert scheme

2.1. Disconnected, pointed version

3. Modifying Brill-Noether, Case (i): separating nodes

3.1. Construction

3.2. Interpretation

3.3. Local forms

3.4. Case of multiples seps

4. Modifying Brill-Noether, case (ii): disjoint separating binodes

4.1. Azimuthal curves : Single binode

4.2. Modifying Hilb

Date: November 2, 2018.

1991 Mathematics Subject Classification. 14N99, 14H99.

Key words and phrases. Hilbert scheme, stable curve, hyperelliptic curve. 
4.3. Normal bundles 23

4.4. Modifying Brill-Noether $\quad 24$

\begin{tabular}{lll} 
4.5. & Interpretation & 27 \\
\hline
\end{tabular}

4.6. Case of multiple disjoint biseps

Part 2. General case $\quad 29$

5. Modifying Brill-Noether: polyseparators $\quad 30$

5.1. S-Stratified blowup $\quad 30$

5.2. Normal blowup 31

5.3. Azimuthal blowup $\quad 33$

5.4. Azimuthal Hilb 35

6. Azimuthal Brill Noether and sepcanonical system $\quad 38$

6.1. Azimuthal Hodge bundle $\quad 38$

6.2. Comparison 39

6.3. Main results $\quad 40$

7. Excess degeneracy and fundamental class $\quad 42$

7.1. Excess Porteous $\quad 42$

7.2. Fundamental class of the hyperelliptics $\quad 43$

8. Azimuthal intersection theory $\quad 46$

8.1. Chern classes of azimuthal bundles $\quad 46$

8.2. Hyperelliptic class in genus 4

8.3. W-bundles 54

References

\section{INTRODUCTION}

Our main aim is to prove Theorem 7.2 below, which is a precise version of the following

Main Theorem (First approximation). Given a family X/B of stable curves, there is a bundle map over an explicit birational modification of its second symmetric product, whose degeneracy locus consists of the closure of the hyperelliptic locus, which is reduced of the expected dimension, plus an explicit and computable excess locus. In this way the fundamental class of the closure of the hyperelliptic locus can be computed as an element of the Mumford tautological ring.

This paper is a continuation of our study of finite subschemes of families of nodal-or-smooth curves (see e.g. [15], [18], [17]). Technically, our aim is to introduce a new tool in the global and enumerative geometry of the moduli space $\overline{\mathcal{M}}_{\mathrm{g}}$ of stable curves: modified Hodge bundles. The rationale for these is a pervasive problem which has long stood in the way of applying 'classical' methods to $\overline{\mathcal{M}}_{\mathrm{g}}$ : that equations (e.g. degeneracy conditions for $g_{d}^{r \prime}$ s) describing geometry on smooth curves become excessively degenerate on singular, especially reducible nodal curves, and accordingly fail to define a limit, in any good sense, of the appropriate locus (e.g. $g_{d}^{r}$ locus) on a smooth curve. Accordingly, most recent work on $\overline{\mathcal{M}}_{\mathrm{g}}$ has focused on extrinsic, GromovWitten type methods, studying maps of curves to other varieties; see [19] for a survey of some of this work and references. 
Nevertheless, this paper represents the beginning of an attempted attack on the aforementioned excess degeneracy problem, based grosso modo on resolving the excess through boundary modifications of Hodge bundles. This approach has its roots in the work of Harris and Mumford [9] on the Kodaira dimension of $\overline{\mathcal{M}}_{\mathrm{g}}$, especially their computation of the fundamental class of the closure of the divisor of curves carrying a $g_{\frac{g+1}{2}}^{1}$. The basic older insight is that the appropriate boundary object corresponding to a linear system is a collection of linear systems on components, or rather certain subcurves, of the boundary curve. The new 'twist' (double-entendre) is that those systems can be accessed via a suitable map of vector bundles.

Specifically, we are concerned here with $g_{2}^{1}$ 's, i.e. the locus of smooth hyperelliptic curves and its closure in $\overline{\mathcal{M}}_{\mathrm{g}}, g \geq 3$, viewed via degeneracy (non-very ampleness) of the canonical system. The usual description in the smooth case is in terms of the 'degree-2 Brill-Noether map' (evaluation map)

$$
\phi: \mathbb{E} \rightarrow \Lambda_{2}(\omega)
$$

where $\mathbb{E}$ is the Hodge bundle and $\Lambda_{2}(\omega)$ denotes the tautological bundle of rank 2 associated to the relative canonical bundle, defined over the degree- 2 relative Hilbert scheme of the universal family (which for smooth curves coincides with the relative symmetric product). Precisely, the degeneracy locus of $\phi$ consists of 'hyperelliptic pairs' $(C, \mathfrak{a})$ where $C$ is a hyperelliptic curve and $\mathfrak{a}$ is a divisor in its unique $g_{2}^{1}$.

Now all of the above data, including $\phi$, extend over $\overline{\mathcal{M}}_{\mathrm{g}}$ and its associated relative Hilbert scheme. But the degeneracy locus of the extended map is not the closure of the locus of hyperelliptic pairs. It contains, e.g. pairs $\left(C_{1} \cup C_{2}, \mathfrak{a}\right)$ where $C_{1} \cup C_{2}$ is a reducible stable curve and $\mathfrak{a}$ is a hyperelliptic divisor on $C_{1}$, which cannot be the limits of a smooth hyperelliptic curve. In essence, this issue is what this paper is about 1 The basic idea is an obvious one: enlarge $\mathbb{E}$ at the boundary by allowing (carefully controlled) poles, so that $\phi$ remains defined but has smaller degeneracy locus, because it effectively accesses a larger linear system. More precisely, given a family $X / B$ of stable curves of genus $g \geq 3$, the enlargement is accomplished via suitable echelon modifications (a generalizations of the familiar elementary modifications, see [16]) along certain boundary divisors. These divisors are associated to the separating nodes and separating pairs of nodes (binodes); the latter case requires blowing up the Hilbert scheme. The ultimate result is the following (see Theorems 6.1 and 7.2 for the precise statements)

Main Theorem (Second approximation). There is a bundle ${ }_{a} \mathbb{E}$ called the azimuthal Hodge bundle, which is an echelon modification of the Hodge bundle defined over a blowup $X_{B}^{\{2\}}$ of the relative Hilbert scheme $X_{B}^{[2]}$, together with a mapping, called the azimuthal Brill-Noether map

$$
{ }_{\mathfrak{a}} \phi:{ }_{\mathfrak{a}} \mathbb{E} \rightarrow \Lambda_{2}(\omega)
$$

whose degeneracy scheme is the union of a lift of the hyperelliptic locus and the locus of schemes supported on some separating node. Via the excess Porteous formula, the fundamental class of the lifted hyperelliptic locus can be computed as an element of the Mumford tautological ring.

\footnotetext{
${ }^{1}$ The presence of extraneous, often excessive, boundary components is a difficulty in Gromov-Witten theory as well.
} 
The contribution to the degeneracy locus from the locus of schemes supported at nodes can be easily computed using residual intersection theory; moreover, its image on $B$ vanishes, essentially because the dualizing sheaf restricts to the structure sheaf on a node (see Cor. 7.3). Therefore, the class of the hyperelliptic locus can be computed by the Theorem using Porteous's formula.

To be fair, the phrase 'can be computed' as used above should be understood, for $g \geq 4$, in the sense of 'reduces to routine, if tedious, calculations'; these calculations can be handled in principle by Gwoho Liu's Macnodal program (see [17]), though some of the details have yet to be worked out.

It should be mentioned that in the case of genus 3, the Brill-Noether map as is has some 'extraneous' (non-hyperelliptic) degeneracy loci, but these are not 'excessive' in dimension, and their contribution can be easily computed, leading to a computation of the hyperelliptic class in $\overline{\mathcal{M}}_{3}$, confirming a formula of Harris-Mumford. See [1], [3] or [17], $\S 4.5$. The genus- 4 case is the first involving excessive degeneration of Brill Noether. It is discussed in detail in 8.2 , where we recover a formula of Faber-Pandharipande ( [6], Prop. 5).

The azimuthal Brill-Noether map is related to a new geometric structure on the boundary, encoded in the sepcanonical system on boundary curves. This is a collection of (usually incomplete) linear systems on certain subcurves ('2-separation components'); on a given subcurve $Y$, the sepcanonical system is a twist of the canonical system of $Y$ which reflects the geometry of $X$ as a whole. Then on subschemes of $Y$, the azimuthal Brill-Noether map of $X$ is 'essentially' the evaluation map associated to the sepcanonical system.

This paper is divided in two parts. Part 1 culminates in the proof of the Main Theorem for curves of semicompact type, i.e. those whose dual graph contains no circuits of size $>2$. Part 2 extends the result to the general case. See the introductions to each part for further organizational details.

Further developments. The methods of this paper seem to extend to the case of pencils (1dimensional systems) of degree $m>2$. This corresponds to studying the submaximal-rank locus or maximal minors ideal of the Brill-Noether map, and involves two main new steps (the details will be pursued elsewhere):

(i) modifying the tautological bundle as well as the Hodge bundle;

(ii) performing bundle modifications on the intermediate boundary components, e.g. those birational to $X_{1}^{(i)} \times X_{2}^{(m-i)}$, for a compact-type boundary curve $X_{0}=X_{1} \cup X_{2}, i=0, \ldots, m$, rather than just the extremal components (where $i=0$ or $m$ ). These modifications eliminate extraneous boundary components.

Being able to perform these bundle modifications, especially for curves with complicated dual graph, would require blowing up the Hilbert scheme, extending the azimuthal modifications considered here.

In the case of 2-dimensional systems, corresponding to the sub-submaximal rank (corank 2 ) locus or submaximal minors ideal of Brill-Noether, extraneous but nonexcessive components arise, and it appears the present methods may apply. Going beyond 2-dimensional systems however gives rise to extraneous and excessive boundary components, and it's not clear how to account for their contributions.

See [6] for another approach, based on Gromov-Witten theory, to computing the fundamental class of hyperelliptic and similar loci related to maps of curves to $\mathbb{P}^{1}$. 
Acknowledgment. I thank the referee for his very careful reading of the paper and highly detailed comments and corrections, including his insistence that we reproduce the Faber- Pandharipande formula in genus 4; these have resulted in a much improved paper. I thank Ann Kostant for her patient and determined assistance.

\section{Part 1. Semicompact type}

This part is mainly devoted to proving the Main Theorem in the case of curves of semi-compact type (defined below); however, some topics are developed in greater generality for use in the general case, to be completed in Part 2 (as well as in potential further applications). In \$1 we review some standard facts about smooth hyperelliptic curves and derive normal form for some objects associated to them, such as the Brill-Noether map.

In $\$ 2$ we review some constructions and properties of Hilbert schemes of curves in the very special case of degree 2.

The core of the paper begins in $\$ 3$, which constructs and studies the modified Hodge bundle in the case of a separating node, using an appropriate echelon modification. The object that appears on the boundary turns out to be closely related to sepcanonical systems.

$\$ 4$ extends the modified Brill-Noether map, first to the case of a single separating binode, then more generally to a disjoint collection of separating nodes and binodes. The binode case, because it occurs in codimension 2, requires a blowup of the Hilbert scheme that we call an azimuthal modification. This amounts to adding some tangential data, called an azimuth, at the binode. On the modified Hilbert scheme, a modified Brill-Noether map can be constructed largely as in the separating node case, again leading to an object closely related to the sepcanonical system. We then prove a provisional form of our main theorem, stating that for curves of 'semi-compact type', i.e. whose dual graph has no cycles of size $>2$, the degeneracy locus of the modified Brill-Noether map consists of an appropriate lift of the hyperelliptic locus, plus the locus of all schemes supported on some separating nodes.

In Part 2, we will extend the latter result to general stable curves, derive a formula for the fundamental class of the stable hyperelliptic locus, and study intersection theory on the azimuthal modification of the Hilbert scheme.

0.1. Riemann-Roch without denominators for anti-self-dual. The purpose of this brief section is to point out that Fulton's Riemann-Roch without [integer] denominators (see [7], Ch. 15) can be simplified in the case of Anti-Self-Dual bundles (defined below), so as to eliminate (characteristic class) denominators other than those of the form $1+D, D=$ divisor, which are easy to invert. These results will be used in $\$ 8.1$

A vector bundle $E$ on a scheme $Y$ is said to be anti-self-dual or ASD if

$$
c(E) c\left(E^{\vee}\right)=1,
$$

where $E^{`}$ denotes the dual bundle.

Example 0.1. As importantly observed by Mumford [12], the Hodge bundle $\mathbb{E}_{g}$ on $\overline{\mathcal{M}}_{\mathrm{g}}$ has the ASD property. Consequently, the pullback of $\mathbb{E}_{g}$ by any map $Y \rightarrow \overline{\mathcal{M}}_{\mathrm{g}}$ also has the ASD property.

Let $D$ be a Cartier divisor on a variety $Y$, let $i_{D}: D \rightarrow Y$ be the inclusion map, and let $E$ be a vector bundle on $D$. The ASD property for a bundle $E$ allows us to avoid the computationally 
unwieldy process of dividing by $c(E)$. Therefore, computations involving the Riemann-Roch without denominators ( [7], §15.3), even in the divisor case, are simplified when the bundle is ASD because rather than divide by $c(E(-D))$ we need only divide by powers of $c\left(\mathcal{O}_{D}(-D)\right)=$ $1-[D]_{D}$. To elaborate, define a polynomial $Q$ in Chern classes of a rank-e bundle $E$ and a line bundle $L$ by

$$
c(E \otimes L)=c(E)+[L] Q(L, E)
$$

or explicitly,

$$
\begin{aligned}
Q(L, E) & =\sum_{p=0}^{e-1} \sum_{i=0}^{p}\left(\begin{array}{c}
e-i \\
p+1-i
\end{array}\right) c_{i}(E) c_{1}(L)^{p-i} \\
& =e+\left(\begin{array}{l}
e \\
2
\end{array}\right) c_{1}(L)+(e-1) c_{1}(E)+\left(\begin{array}{l}
e \\
3
\end{array}\right) c_{1}^{2}(L)+\left(\begin{array}{c}
e-1 \\
2
\end{array}\right) c_{1}(L) c_{1}(E)+(e-2) c_{2}(E)+\ldots
\end{aligned}
$$

Note that

$$
Q(L, E)=Q\left(L^{\vee}, E \otimes L\right) .
$$

The Riemann-Roch without denominators ( [7], Example 15.3.4) states that, for any locally free $\mathcal{O}_{D^{-}}$sheaf $E$, we have

$$
c\left(i_{D *}(E)\right)=1+i_{D *}\left(\frac{Q(-D, E)}{c\left(E \otimes \mathcal{O}_{D}(-D)\right)}\right) .
$$

Then from the definition of ASD, we conclude directly:

Proposition 0.2. Notations as above, for E ASD of rank e on the divisor D, and any Cartier divisor $G$ on $D$, we have

$$
c\left(i_{D *}(E(G))\right)=1+i_{D *}\left((1+G-D)^{-2 e} Q(-D, E(G)) c\left(E^{\vee}(G-D)\right)\right) .
$$

Example 0.3. When $G=D$, the above simplifies nicely, using (0.1.3), to

$$
\begin{aligned}
c\left(i_{D *}(E(D))\right) & =1+i_{D *}\left(Q(D, E) c\left(E^{\vee}\right)\right) \\
& =1+i_{D *}\left(\sum_{j=0}^{e} \sum_{p=0}^{e-1} \sum_{i=0}^{p}(-1)^{j}\left(\begin{array}{c}
e-i \\
p+1-i
\end{array}\right) c_{i}(E) c_{j}(E) D^{p-i}\right) .
\end{aligned}
$$

Example 0.4. Let $Y=\overline{\mathcal{M}}_{\mathrm{g}}$ and let $D$ be the divisor $\overline{\mathcal{M}}_{h, 1} \times \overline{\mathcal{M}}_{g-h, 1}$, with conormal bundle $\psi=$ $\psi_{h} \otimes \psi_{g-h}$, the product of the respective cotangent classes. Let $\mathbb{E}_{h}$ be the pullback of the rank- $h$ Hodge bundle to $D$, which is ASD as vector bundle over $D$ because the Hodge bundle itself is ASD as vector bundle over $\overline{\mathcal{M}}_{h}$. Then for a divisor class $G$ on $D$,

$$
c\left(i_{D *}\left(\mathbb{E}_{h} \otimes G\right)\right)=1+i_{D *}\left(\frac{Q\left(\psi, \mathbb{E}_{h} \otimes G\right) c\left(\mathbb{E}_{h}{ }^{2} \otimes G \otimes \psi\right)}{(1+G+\psi)^{2 h}}\right) .
$$

Corollary 0.5. Assumptions as above, let $Z \subset D$ be a subvariety such that $c_{1}(\mathcal{O}(D)) \cap[Z]=0$ and let $F$ be any vector bundle on $Y$. Then: (i)

$$
c\left(i_{D *}(E \otimes F)\right) \cdot Y Z=1
$$


(ii) for any line bundle $M$ on $D$,

$$
c\left(i_{D *}(M)\right) \cdot \gamma Z=1 .
$$

Proof. (i) Because $i_{D *}\left(E \otimes_{D} F\right)=i_{D *}(E) \otimes_{Y} F$, we may assume $F=\mathcal{O}_{Y}$. Then the assertion is obvious from the Proposition above, plus the standard fact that for any class $a$ on $D$,

$$
i_{Z, Y}^{*} i_{D *}(a)=i_{Z, D}^{*}(a \cdot[D]) .
$$

(ii) In this case, Fulton's example reads

$$
c\left(i_{D *}(M)\right)=1+i_{D *} \frac{1}{1+[M]+[L]}, L=\mathcal{O}_{D}(-D) .
$$

Then our assertion follows easily from (0.1.7) above.

\section{LOCUS OF SMOOTH HYPERELLIPTICS: A REVIEW}

The purpose of this section is to review some elementary facts about smooth hyperelliptic curves and the locus they make up in a family of smooth curves, especially the normal bundle to this locus.

In general, given a versal family $\pi: X \rightarrow B$ of stable, generically smooth, curves, we let $\mathcal{H} \mathcal{E}_{B} \subset B$ denote the closure of the locus of smooth hyperelliptic curves, which is of virtual codimension equal to $g-2$. We also denote by $\mathcal{H E}_{B}^{2} \subset X_{B}^{[2]}$ the closure of the locus of schemes invariant by the hyperelliptic involution, i.e. fibres of the canonical mapping. This has virtual codimension $g-1$. The fibre of $\mathcal{H E}_{B}^{2}$ over an interior point $b \in B$ is either empty, if $X_{b}$ is nonhyperelliptic, or equal to the target $\mathbb{P}^{1}$ of the hyperelliptic pencil, otherwise. We will denote by $\mathcal{H} \mathcal{E}_{B}^{1} \subset \mathcal{H E}_{B}^{2}$ the sublocus consisting of length-2, 1-point schemes, i.e. the (schematic) intersection of $\mathcal{H E}^{2}$ with the diagonal divisor $\Gamma=\Gamma^{[2]} \simeq X$ or equivalently, the locus of ramification points of the hyperelliptic map, i.e. Weierstrass points. Because the ramification is simple, the intersection is transverse and $\mathcal{H E}_{B}^{1} \rightarrow \mathcal{H E}_{B}$ is étale. We let $\mathbb{E}$ denote the Hodge bundle on $B$, $\mathbb{E}=\pi_{*} \omega, \omega=\omega_{X / B}$.

1.1. Normal bundle. Let $X$ be a smooth hyperelliptic curve with hyperelliptic map

$$
f: X \rightarrow \mathbb{P}^{1}
$$

and $\eta=f^{*} O(1)$ the hyperelliptic bundle and $\beta \subset X$ the ramification divisor, which is reduced of degree $2 g+2$. Then deformations of the pair $(X, f)$ are unobstructed and parametrized by $H^{0}\left(N_{f}\right)$, which fits in an exact sequence

$$
0 \rightarrow H^{0}\left(f^{*}\left(\theta_{\mathbb{P}^{1}}\right)\right) \rightarrow H^{0}\left(N_{f}\right) \rightarrow H^{1}\left(\theta_{X}\right) \rightarrow H^{1}\left(f^{*}\left(\theta_{\mathbb{P}^{1}}\right)\right) \rightarrow 0
$$

and $f^{*}\left(\theta_{\mathbb{P}^{1}}\right)=\eta^{2}$. Then the first group coincides with $\operatorname{Sym}^{2}\left(H^{0}(\eta)\right)$, which corresponds to reparametrizations of the target $\mathbb{P}^{1}$, while the last group is dual to $H^{0}\left(\omega \otimes \eta^{-2}\right)=H^{0}\left(\omega^{2}(-\beta)\right)$ which is $g-2$-dimensional. This implies that in any versal family $X / B$, the hyperelliptic locus $\mathcal{H E}_{B} \subset B$ is smooth of codimension $g-2$. Moreover, because the Weierstrass point locus $\mathcal{H} \mathcal{E}_{B}^{1} \subset$ $X$ is étale over $\mathcal{H} \mathcal{E}_{B}$, it too is smooth. 
1.2. Normal forms. We study the locus of hyperelliptics (and related loci) in a family of smooth (non-pointed) curves. Consider a family $X / B$ of smooth curves. We note that because of the existence of a tautological family of curves over $B$, the degree of the natural map $B \rightarrow \mathcal{M}_{g}$ is at least 2 near any hyperelliptic curve. We assume the family is locally versal, hence this map is ramified only over the hyperelliptic locus and other loci of curves with automorphisms. Therefore $\mathcal{H E}_{B}$ is smooth of codimension $g-2$ in $B$ (of course $\mathcal{M}_{g}$ is singular along its image). Then $\mathcal{H E}_{B}^{2} \subset X_{B}^{(2)}$ is just the degeneracy (rank-1) locus of the natural evaluation map that we will call the (degree-2) Brill-Noether map

$$
\phi: \pi^{(2) *} \mathbb{E} \rightarrow \Lambda_{2}(\omega) .
$$

Here $\Lambda_{2}(\omega)$ is the 'secant bundle' which in the general case of stable curves is defined on the relative Hilbert scheme $X_{B}^{[m]}$ (see $\S_{2}$ ).

Our purpose here is to give a normal form for the Brill-Noether map $\phi$ and an 'augmented' analogue, especially in a neighborhood of a hyperelliptic curve. Let $\left(X_{0}, \theta_{0}\right)$ be a hyperelliptic pair or 'pointed hyperelliptic curve', i.e. $X_{0}$ is hyperelliptic and $\theta_{0}$ is a Weierstrass point on it. We work locally on the degree-2 Hilbert scheme $X_{0}^{[2]}=X_{0}^{(2)}$, with the tautological rank-2 bundle $\Lambda_{2}(\mathcal{O})$, at the scheme $2 \theta_{0}$ (see $₫ 2$ for a review). Let $s_{0}, \ldots, s_{g-1}$ be a basis for $H^{0}\left(\omega_{X_{0}}\right)$ such that

$$
\operatorname{ord}_{\theta_{0}}\left(s_{i}\right)=2 i
$$

i.e. locally at $\theta_{0}$ with local coordinate $x, s_{i} \sim x^{2 i}$. Local coordinates for $X_{0}^{(2)}$ near $2 \theta_{0}$ are $\sigma_{1}=$ $x_{1}+x_{2}, \sigma_{2}=x_{1} x_{2}$ where $x_{i}=p_{i}^{*} x$. Set

$$
e_{i}=\Lambda_{2}\left(x^{i}\right)
$$

Then $e_{0}, e_{1}$ is a local frame for $\Lambda_{2}(\mathcal{O})$ and we have

$$
e_{i+1}=\sigma_{1} e_{i}-\sigma_{2} e_{i-1}, i \geq 1 .
$$

It is easy to check from (1.2.3) that if we write $e_{i}=a_{0 i} e_{0}+a_{1 i} e_{1}, i \geq 2$, then

$$
\begin{array}{r}
a_{0 i} \equiv 0 \quad \bmod \sigma_{2} \\
a_{1 i} \equiv 0 \quad \bmod \sigma_{1}, i \text { even. }
\end{array}
$$

In terms of these data, the Brill-Noether map is represented by the $2 \times g$ matrix

$$
\Lambda=\Lambda_{2}\left(s_{0}, \ldots, s_{g-1}\right)=\left[\begin{array}{cccc}
1 & -\sigma_{2} & \sigma_{2}\left(\sigma_{2}-\sigma_{1}^{2}\right) & \ldots \\
0 & \sigma_{1} & \sigma_{1}\left(\sigma_{1}^{2}-2 \sigma_{2}\right) & \ldots
\end{array}\right]
$$

It is easy to see that all entries of the 1st (resp. 2nd) row beyond the 1st column are divisible by $\sigma_{2}$ (resp. $\sigma_{1}$ ). Consequently the ideal of $2 \times 2$ minors of $\Lambda$ is $\sigma_{1}$, i.e the equation of the graph of hyperelliptic involution.

To deal with equations for hyperelliptic pairs we consider analogously the augmented BrillNoether map, which is the analogous map for the line bundle $\omega\left(2 \theta_{0}\right)$. Note that for any smooth pair $(X, \theta), \omega_{X_{0}}\left(2 \theta_{0}\right)$ is base-point free, $(g+1)$-dimensional and ramified at $\theta_{0}$, and $\left(X_{0}, \theta_{0}\right)$ is hyperelliptic if and only if the mapping associated to $\left|\omega_{X_{0}}\left(2 \theta_{0}\right)\right|$ fails to be an isomorphism off $\theta_{0}$, in which case it is actually composed of the hyperelliptic involution. If $\left(X_{0}, \theta_{0}\right)$ is hyperelliptic, 
we may choose a basis (s.) for $H^{0}\left(\omega_{X_{0}}\left(2 \theta_{0}\right)\right)$ so that $\operatorname{ord}_{\theta}\left(s_{i}\right)=2 i, 0 \leq i \leq g$ so the associated augmented $2 \times(g+1)$ Brill-Noether matrix has the form similar to (1.2.4)

$$
\Lambda^{+}=\Lambda_{2}\left(s_{0}, \ldots, s_{g}\right)=\left[\begin{array}{cccc}
1 & -\sigma_{2} & \sigma_{2}\left(\sigma_{2}-\sigma_{1}^{2}\right) & \ldots \\
0 & \sigma_{1} & \sigma_{1}\left(\sigma_{1}^{2}-2 \sigma_{2}\right) & \ldots
\end{array}\right]
$$

By contrast, when $(X, \theta)$ is non-hyperelliptic, the sequence of vanishing orders at $\theta$ for $\omega(2 \theta)$ starts $(0,2,3, \ldots)$, so the augmented Brill-Noether starts

$$
\left[\begin{array}{cccc}
1 & * & * & \cdots \\
0 & \sigma_{1} & \sigma_{1}^{2}-\sigma_{2} & \cdots
\end{array}\right]
$$

So in that case the ideal of 2-minors of this is clearly $\left(\sigma_{1}, \sigma_{2}\right)$, the ideal of the point $2 \theta_{0} \in X_{0}^{(2)}$.

Now suppose $X_{0}$ varies in a versal family $\left(X_{0} \subset X\right) /(0 \in B)$ and admits a section $\theta$ (not considered part of the data and subject to change), whose value over $0 \in B$ is a Weierstrass point $\theta_{0}$ on $X_{0}$, and extend (s.) to a basis $\tilde{s}_{i}$ of the Hodge bundle $\mathbb{E}$ over $B$. In terms of a local fibre coordinate, we can write, after a suitable change of basis

$$
\tilde{s}_{i}=x^{2 i}+z_{i} x^{2 i-1}+\sum_{i \leq j \leq 2 i-2} w_{i j} x^{j}, i=1, \ldots, g-1
$$

where the $z_{i}, w_{i j} \in \mathfrak{m}_{0, B}$. By suitably changing the section $\theta$, or equivalently, changing the fibre coordinate $x$ based at $\theta$ to $x-z_{1} / 2$, we may assume $z_{1}=0$, i.e. $s_{1}=x^{2}$. This ensures that $\theta$ is in the ramification locus of the map to $\mathbb{P}^{1}$ determined by $s_{0}, s_{1}$. As long as $X$ varies in the hyperelliptic locus $\mathcal{H E}_{B}$, the latter ramification locus coincides locally with the locus of Weierstrass points, which itself is étale over $\mathcal{H} \mathcal{E}_{B}$. Thus, $\theta$ remains a Weierstrass point in any deformation of $X$ as hyperelliptic curve. Then for $i>1$, we may inductively subtract off a suitable $\mathcal{O}_{B}$-linear combination of the $s_{h}, h<i$ to arrange that $w_{i j}=0$ for $j$ even. Consequently, note that the schematic condition defining the locus $\mathcal{H E}_{B} \subset B$ is now precisely

$$
z_{2}=\ldots=z_{g-1}=0 .
$$

Indeed this vanishing condition is equivalent to $h^{1}(\mathcal{O}(2 \theta))=h^{0}(\omega(-2 \theta) \geq g-1$, i.e. by Riemann-Roch, $h^{0}(\mathcal{O}(2 \theta)) \geq 2$. The fibre of $\mathcal{H E}_{B}^{2} \rightarrow \mathcal{H E}_{B}$ is the graph of the hyperelliptic involution on a given curve, which for suitable coordinates is given by the 'antidiagonal' $\sigma_{1}=0$ (which is transverse to the diagonal, due to the fact that the involution has simple fixed points). Because $\mathcal{H E}_{B} \subset B$ is a smooth subvariety of codimension $g-2, z_{2}, \ldots, z_{g-2}$ are regular parameters near 0.

Now because the $w_{i j}$ vanish on the hyperelliptic locus, we can write

$$
w_{i j}=\sum_{k=2}^{g-1} u_{i j k} z_{k}
$$

Plugging this into the Brill-Noether matrix for the family, we obtain

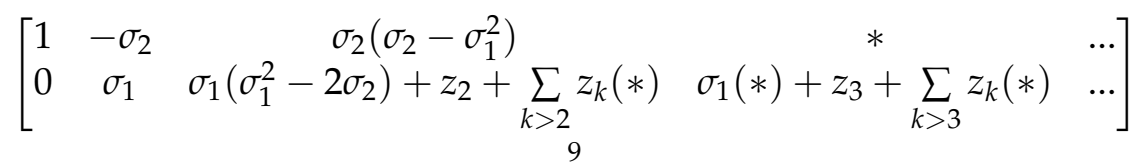


which is column-equivalent to

$$
\left[\begin{array}{ccccc}
1 & * & * & * & \cdots \\
0 & \sigma_{1} & z_{2} & z_{3} & \cdots
\end{array}\right]
$$

The degeneracy locus of this is precisely

$$
\mathcal{H E}_{B}^{2}=\left\{\sigma_{1}=z_{2}=\ldots=z_{g-1}=0\right\} \subset X_{B}^{(2)} .
$$

1.3. Pointed case. The case of the pointed hyperelliptic locus, i.e. the locus of hyperelliptic pairs $(X, \theta)$ is similar, using the augmented Brill-Noether map. Thus let $\left(X_{B_{1}}, \theta_{B_{1}}\right)$ be a versal family of pointed smooth curves parametrized by $B_{1}$, where we may assume $B_{1}$ itself is the total space of a family $X_{B}$ over $B$, with the extra parameter specifying the value of the section $\theta$. Then, working analogously with the system $\omega(2 \theta)$, we may assume

$$
\begin{array}{r}
s_{0}=1, s_{1}=x^{2}, \\
s_{i}=x^{2 i}+z_{i} x^{2 i-1}+\sum_{j=i+1}^{2 i-2} w_{i j} x^{j}, i=2, \ldots, g
\end{array}
$$

where $w_{i j}=0$ for $j$ even. Here $z_{1}$ is a vertical coordinate of $B_{1} / B$, i.e. a fibre coordinate. As before, the ideal of the locus of hyperelliptic pairs $\mathcal{H E}_{B}^{1} \subset B_{1}$, i.e. the locus of pairs $(X, \theta)$ where $h^{0}\left(\omega_{X}(-2 \theta)\right) \geq g-1$, is generated by the $z^{\prime}$ s, so we may assume the $w^{\prime}$ s are in the ideal generated by the $z^{\prime}$ s. Now we have an augmented Brill-Noether map over $X_{B_{1}}^{(2)}$ :

$$
\left(\pi^{(2)}\right)^{*} \pi_{*}(\omega(2 \theta)) \rightarrow \Lambda_{2}(\omega(2 \theta))
$$

The matrix relative to the $s$. basis above and the usual $e_{0}, e_{1}$ basis on the target, known as the augmented Brill-Noether matrix, takes the form

$$
\Lambda^{+}=\left[\begin{array}{ccccc}
1 & * & * & \cdots & * \\
0 & \sigma_{1} & z_{2} \sigma_{2} & \cdots & z_{g} \sigma_{2}
\end{array}\right]
$$

The degeneracy locus of this in $X_{B}^{(2)}$ is the schematic union of the section $2 \theta$, with ideal $\left(\sigma_{1}, \sigma_{2}\right)$, and the locus of the hyperelliptic involution, lying over the locus of hyperelliptic pairs in $B$, with ideal $\left(\sigma_{1}, z_{2}, \ldots, z_{g}\right)$. Again by our assumptions, these are regular parameters at $(0,2 \theta)$.

\section{GOOD FAMILIES AND THEIR HILBERT SCHEME}

For more information on Hilbert schemes of families of nodal curves, see [13] or [17]. Consider a proper family $\pi: X \rightarrow B$ of connected nodal curves of genus $g$. Fix a fibre node $\theta$ of $X / B$, with corresponding boundary divisor $\partial=\partial_{\theta} \subset B$. At least locally in $X$ near $\theta$, the boundary family $X_{\partial}$ splits:

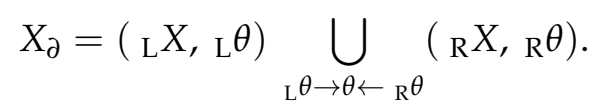

In the case $\theta$ is separating- the case of principal interest here- the splitting is defined locally in $B$, and even globally in $B$ if $g\left({ }_{\mathrm{L}} X\right) \neq g\left(\mathrm{R}_{\mathrm{R}} X\right)$, e.g. if $g$ is odd. We may call ${ }_{\mathrm{L}} X,{ }_{\mathrm{R}} X$ the left and right sides of $\theta$ respectively. The choice of left and right sides of $\theta$ is called an orientation. We call the boundary component $\partial$ decomposable if

$$
\partial=\underset{L}{L} \partial \times_{R} \partial
$$


where $\left({ }_{*} X,{ }_{*} \theta\right) /{ }_{*} \delta$ is a family of stable pointed curves of genus ${ }_{*} g, *=L, R,{ }_{L} g+{ }_{\mathrm{R}} g=g$. This is certainly true for the universal family over $\overline{\mathcal{M}}_{\mathrm{g}}$ and suitably chosen base-changes over it. The decomposability assumption is not essential for our basic constructions, however.

Now consider the length-2 Hilbert scheme $X_{B}^{[2]}$ near the boundary component $\partial$. Its boundary part has the form

$$
\left({ }_{\mathrm{L}} X\right)_{\partial}^{[2]} \cup{ }_{\mathrm{L} R} X \cup\left({ }_{\mathrm{R}} X\right)_{\partial}^{[2]}
$$

with ${ }_{\mathrm{L} R} X \rightarrow{ }_{\mathrm{L}} X \times{ }_{\partial}{ }_{\mathrm{R}} X$ the blowing-up of $\left({ }_{\mathrm{L}} \theta,{ }_{\mathrm{R}} \theta\right)$, with exceptional divisor

$$
R_{\theta}=\mathbb{P}\left({ }_{\mathrm{L}} \psi \oplus{ }_{\mathrm{R}} \psi\right),_{*} \psi=T_{*}^{*} \theta_{, *} X / \partial .
$$

The locus $R_{\theta}$ parametrizes relative length-2 subschemes of $X$ supported on $\theta$. It is a $\mathbb{P}^{1}$-bundle over $\partial$ admitting a pair of disjoint sections ${ }_{*} Q:=\mathbb{P}\left({ }_{*} \psi\right)=R_{\theta} \cap\left({ }_{*} X\right)_{B}^{[2]}, *=L, R$. Moreover, if $* \dagger$ denotes the mirror of $*=L, R$,

$$
\left({ }_{*} X\right)_{\partial}^{[2]} \cap{ }_{\mathrm{L} R} X={ }_{*} X \times{ }_{* \dagger} \theta, *=L, R ;\left({ }_{\mathrm{L}} X\right)_{\partial}^{[2]} \cap\left({ }_{\mathrm{R}} X\right)_{\partial}^{[2]}=\varnothing .
$$

Now working locally, assume the family is given by the standard form $x y=t$ with $x, y$ regular parameters on $X$ and $t$ a local defining equation for the Cartier divisor $\delta$ on $B$. Set $x_{i}=p_{i}^{*} x, i=$ 1,2 (a function on the Cartesian square) and similarly $y_{i}$. The elementary symmetric functions $\sigma_{1}^{x}, \sigma_{2}^{x}$ (resp. $\sigma_{1}^{y} \sigma_{2}^{y}$ ) are functions on the symmetric product. Then near the finite part of $R_{\theta}$, i.e. off ${ }_{\mathrm{R}} Q$, we get regular parameters on $X_{B}^{[2]}$

$$
\sigma_{1}=\sigma_{1}^{x}, \sigma_{2}=\sigma_{2}^{x}, u=y_{1} / x_{2}=y_{2} / x_{1}=\left(\sigma_{1}^{y}\right) /\left(\sigma_{1}^{x}\right),
$$

with $\sigma_{2}, u$ being defining equations, respectively, for ${ }_{\mathrm{L} R} X,\left({ }_{\mathrm{L}} X\right)_{\partial}^{[2]}$, while $\sigma_{1}, \sigma_{2}$ together define $R_{\theta}$. Similarly, off ${ }_{L} Q$, we have $\sigma_{1}^{y}, \sigma_{2}^{y}, v:=1 / u$ as regular parameters. Note that the diagonal is defined here by $\sigma_{1}^{2}-4 \sigma_{2}$ (in either $x$ or $y$ variables in the appropriate open set). This diagonal is isomorphic to the blowup of $X$ in $\theta$, with coordinates $\sigma_{1}, u$.

We want to study the Brill-Noether map

$$
\phi:\left(\pi^{[2]}\right)^{*}(\mathbb{E}) \rightarrow \Lambda_{2}(\omega)
$$

locally over $2 \theta \in X_{B}^{(2)}$ and off ${ }_{L} Q \cup{ }_{\mathrm{R}} Q$. We recall (see (1.2.2)) that $e_{i}=\Lambda_{2}\left(x^{i}\right)$ and $e_{0}, e_{1}$ is a local basis for $\Lambda_{2}\left(\mathcal{O}_{X}\right)$, identified with $\Lambda_{2}(\omega)$. Then note that

$$
\begin{array}{r}
\Lambda_{2}(y)=u\left(\sigma_{1} e_{0}-e_{1}\right), \Lambda_{2}\left(y^{2}\right)=u^{2}\left(\left(\sigma_{1}^{2}-\sigma_{2}\right) e_{0}-\sigma_{2} e_{1}\right), \\
\Lambda_{2}\left(y^{3}\right)=u^{3}\left(\left(\sigma_{1}^{3}-2 \sigma_{1} \sigma_{2}\right) e_{0}+\left(\sigma_{2}-\sigma_{1}^{2}\right) e_{1}\right), \\
\Lambda_{2}\left(y^{i}\right)=u \sigma_{1} \Lambda_{2}\left(y^{i-1}\right)-u^{2} \sigma_{2} \Lambda_{2}\left(y^{i-2}\right) .
\end{array}
$$

This comes from the fact that, on the Cartesian product, the lift $\Lambda_{2}^{\lceil}(y)$ of $\Lambda_{2}(y)$ is given by

$$
\Lambda_{2}^{\lceil}(y)=\left(y_{1}, y_{2}\right)=u\left(x_{2}, x_{1}\right)=u\left(\sigma_{1} e_{0}-e_{1}\right) .
$$

Now assuming $\left(X_{1}, \theta_{1}\right),\left(X_{2}, \theta_{2}\right)$ are both non-hyperelliptic, $\omega_{X_{i}}\left(\theta_{i}\right)$ have vanishing sequences $(1,2, \ldots)$, therefore the matrix of the Brill-Noether mapping with respect to the $e_{0}, e_{1}$ basis on $\Lambda_{2}$ 
and a suitable adapted basis of the Hodge bundle, where the part corresponding to $X_{2}$ is located right of the vertical bar and indexed negatively, has the form

$$
\left[\begin{array}{ccc|ccc}
\ldots & u^{2}\left(\sigma_{1}^{2}-\sigma_{2}\right) & u \sigma_{1} & 0 & -\sigma_{2} & \ldots \\
\ldots & -u^{2} \sigma_{2} & -u & 1 & \sigma_{1} & \ldots
\end{array}\right]
$$

whose degeneracy scheme coincides locally with the schematic union

$$
\operatorname{Zeros}\left(u, \sigma_{2}\right) \cup \operatorname{Zeros}\left(\sigma_{1}, \sigma_{2}\right)=\left({ }_{\mathrm{L}} X \times{ }_{\mathrm{R}} \theta\right) \cup R_{\theta} .
$$

Taking into account the 'opposite' open set containing $Q_{2}$, we see that the degeneracy locus of $\phi$ is

$$
R_{\theta} \cup{ }_{\mathrm{L}} X \times{ }_{\mathrm{R}} \theta \cup{ }_{\mathrm{R}} X \times{ }_{\mathrm{L}} \theta,
$$

i.e. the locus of schemes whose support contains the node $\theta$. In the next section we will describe a modification of $\mathbb{E}$ and $\phi$ that will have a smaller degeneracy locus; e.g. over the general curve, we just get $R_{\theta}$.

Definition 2.1. A family of nodal curves $X / B$ is said to be good if

- every fibre is semi-stable;

- every boundary component with reducible general fibre is decomposable;

- the family is everywhere locally versal: i.e. for every fibre $X_{0}$ with nodes $\theta_{1}, \ldots, \theta_{k}$ the map from the germ of $B$ at 0 to the product of local deformation spaces of $X_{0}$ at $\theta_{1}, \ldots, \theta_{k}$ is smooth.

2.1. Disconnected, pointed version. Let $Y$ be a possibly disconnected nodal curve (over some base) endowed with a collection of disjoint (étale) multisections $p_{1}, \ldots, p_{n}$ with each $p_{i}$ contained in a unique connected component. A separating node of $Y$ is a node $\theta$ whose blowup disconnects some connected component of $Y$ in two components, say ${ }_{\mathrm{L}} Y(\theta), \mathrm{R} Y(\theta)$, and for each $i, p_{i}$ is either contained in or disjoint from ${ }_{\mathrm{L}} Y(\theta)$ (resp. ${ }_{\mathrm{R}} Y(\theta)$ ).

\section{Modifying Brill-Noether, CASE (I): SePARATing NOdeS}

The traditional difficulty with extending the degeneracy-locus description of the hyperelliptic locus (and its analogues) across the boundary stems largely from the presence of 'part dead' sections of the relative canonical, i.e. sections vanishing on some component. In this paper, our approach to resolving this difficulty is to revive the sections that vanish on the part of the curve equal to a side of a node or binode by enlarging the Hodge bundle, i.e. the source of the BrillNoether map; or rather, we enlarge the pullback of the Hodge bundle over the degree-2 Hilbert scheme (or in the next section, a modification thereof). This enlargement is accomplished by appropriate echelon modifications (see [16]) of the Hodge bundle which mirror, and will later be linked (see Theorem 6.1) to the modifications yielding the sepcanonical system, developed in [18]. We begin in this section with the case of a separating node. The more challenging case of a separating binode is taken up in the next section.

3.1. Construction. For now, we work one node at a time. Thus, fix a separating node or 'sep' $\theta$ of the family $\pi: X \rightarrow B$ with corresponding boundary divisor $\partial=\partial_{\theta}$. Set

$$
{ }_{\mathrm{R}} X={ }_{\mathrm{R}} X(\theta),{ }_{\mathrm{L}} X={ }_{\mathrm{L}} X(\theta) .
$$


These are families over $\partial$ and embedded as Cartier divisors on $X$. For now, assume for convenience that these are individually defined, i.e. that $\theta$ is oriented as a sep. The ultimate construction will not depend on the choice of orientation. We will also assume for now that ${ }_{\mathrm{L}} X,{ }_{\mathrm{R}} X$ have respective genera ${ }_{\mathrm{L}} g,{ }_{\mathrm{R}} g \geq 2$.

Define twisted Hodge bundles on B:

$$
\mathbb{E}^{i, j}=\pi_{*}\left(\omega\left(i_{\mathrm{L}} X+j_{\mathrm{R}} X\right)\right) .
$$

For any $i \leq i^{\prime}, j \leq j^{\prime}$, there is a full-rank inclusion $\mathbb{E}^{i, j} \rightarrow \mathbb{E}^{i^{\prime}, j^{\prime}}$. In particular, we consider two chains of full-rank subsheaves

$$
\begin{aligned}
& \mathbb{E}^{-2,0} \rightarrow \mathbb{E}^{-1,0} \rightarrow \mathbb{E}, \\
& \mathbb{E}^{0,-2} \rightarrow \mathbb{E}^{0,-1} \rightarrow \mathbb{E},
\end{aligned}
$$

induced respectively by

$$
\begin{aligned}
& \omega\left(-2_{\mathrm{L}} X\right) \rightarrow \omega\left(-{ }_{\mathrm{L}} X\right) \rightarrow \omega, \\
& \omega\left(-2_{\mathrm{R}} X\right) \rightarrow \omega\left(-{ }_{\mathrm{R}} X\right) \rightarrow \omega .
\end{aligned}
$$

On the boundary, the latter sheaves take the form

$$
\begin{aligned}
& { }_{\mathrm{L}} \omega\left(3{ }_{\mathrm{L}} \theta\right) \cup{ }_{\mathrm{R}} \omega\left(-{ }_{\mathrm{R}} \theta\right) \rightarrow{ }_{\mathrm{L}} \omega\left(2{ }_{\mathrm{L}} \theta\right) \cup{ }_{\mathrm{R}} \omega \rightarrow{ }_{\mathrm{L}} \omega\left({ }_{\mathrm{L}} \theta\right) \cup{ }_{\mathrm{R}} \omega\left({ }_{\mathrm{R}} \theta\right), \\
& { }_{\mathrm{L}} \omega\left(-{ }_{\mathrm{L}} \theta\right) \cup{ }_{\mathrm{R}} \omega\left(3{ }_{\mathrm{R}} \theta\right) \rightarrow{ }_{\mathrm{L}} \omega \cup{ }_{\mathrm{R}} \omega\left(2{ }_{\mathrm{R}} \theta\right) \rightarrow{ }_{\mathrm{L}} \omega\left({ }_{\mathrm{L}} \theta\right) \cup{ }_{\mathrm{R}} \omega\left({ }_{\mathrm{R}} \theta\right),
\end{aligned}
$$

where ${ }_{*} \omega=\omega_{* X / * \delta}, *=L, R$. The maps in the two rows are given, locally near $\theta$, by multiplication by $y, x$ respectively, where $x, y$ are respective local equations for ${ }_{\mathrm{R}} X,{ }_{\mathrm{L}} X$. So these maps are injective on ${ }_{\mathrm{L}} X$ and zero on ${ }_{\mathrm{R}} X$ or vice versa. On fibres, the sheaves involved, other than $\omega={ }_{\mathrm{L}} \omega\left({ }_{\mathrm{L}} \theta\right) \cup{ }_{\mathrm{R}} \omega\left({ }_{\mathrm{R}} \theta\right)$ itself, are base-point free on at least one of ${ }_{\mathrm{L}} X,{ }_{\mathrm{R}} X$, and it is elementary to check that the direct images are locally free and compatible with base-change.

(2)

II NB: the latter assertion would be false for any other twists of $\omega$, e.g. ${ }_{L} \omega\left(4_{L} \theta\right) \cup{ }_{R} \omega\left(-2{ }_{R} \theta\right)$ : this is because $h^{0}\left({ }_{\mathrm{R}} \omega\left(-2{ }_{\mathrm{R}} \theta\right)\right)$ can jump.

Now we shift attention to the Hilbert scheme $X_{B}^{[2]}$. Set ${ }_{*} D={ }_{*} D(\theta)=\left({ }_{*} X\right)_{\delta}^{[2]}$, a Cartier divisor on $X_{B}^{[2]}$. In the local coordinates above, it has equation $u$ for $*=L$ and $v=1 / u$ for $*=R$. Consider the following echelon data $([16], \S 1)$ on $X_{B}^{[2]}$ :

$$
\begin{array}{r}
\chi(\theta)=\left({ }_{\mathrm{L}} \chi(\theta),{ }_{\mathrm{R}} \chi(\theta)\right) \\
{ }_{\mathrm{L}} \chi=\left(\left(\mathbb{E}^{-2,0},{ }_{\mathrm{L}} D\right),\left(\mathbb{E}^{-1,0},{ }_{\mathrm{L}} D\right), \mathbb{E}\right) \\
{ }_{\mathrm{R}} \chi=\left(\left(\mathbb{E}^{0,-2}, 2{ }_{\mathrm{R}} D\right),\left(\mathbb{E}^{0,-1},{ }_{\mathrm{R}} D\right), \mathbb{E}\right) .
\end{array}
$$

These are certainly transverse as ${ }_{\mathrm{L}} D \cap{ }_{\mathrm{R}} D=\varnothing$. Let $\mathbb{E}_{\theta}$ be the associated echelon modification ( [16], §2). Clearly, the Brill-Noether mapping $\phi$ vanishes on $\mathbb{E}^{-1,0}$ over ${ }_{\mathrm{L}} D$, over $\mathbb{E}^{-2.0}$ to order 2 over ${ }_{L} D$ etc. Therefore by the universal property of echelon modifications ( [16], Theorem 2.3), $\phi$ factors through $\mathbb{E}_{\theta}$ :

$$
\left(\pi^{[2]}\right)^{*}(\mathbb{E}) \rightarrow \mathbb{E}_{\theta} \stackrel{\phi_{\theta}}{\rightarrow} \Lambda_{2}(\omega) .
$$

We call $\phi_{\theta}$ the modified Brill Noether map (with respect to the $\operatorname{sep} \theta$ ). 
Remark 3.1. In the event that ${ }_{\mathrm{L}} g=1$, the datum ${ }_{\mathrm{L}} \chi$ above is to be replaced by

$$
{ }_{\mathrm{L}} \chi=\left(\left(\mathbb{E}^{-1,0},{ }_{\mathrm{L}} D\right), \mathbb{E}\right)
$$

and similarly if ${ }_{\mathrm{R}} g=1$.

3.2. Interpretation. We will analyze $\phi_{\theta}$ near ${ }_{\mathrm{L}} D$. Recall from [16], (8), the injective map

$$
\mathbb{E}^{-2,0}\left(2_{\mathrm{L}} D\right) \rightarrow \mathbb{E}_{\theta}
$$

that is an isomorphism over the 'interior' ${ }_{\mathrm{L}} D^{o}$ of ${ }_{\mathrm{L}} D$, i.e.

$$
{ }_{\mathrm{L}} D^{o}={ }_{\mathrm{L}} D \backslash_{\mathrm{L}} X \times{ }_{\mathrm{L}} \theta=\left\{\text { schemes not containing }{ }_{\mathrm{L}} \theta\right\} \text {. }
$$

On the other hand, the inclusion $\omega\left(-2_{\mathrm{L}} X\right) \rightarrow \omega$ induces over $X_{B}^{[2]}$ a map

$$
\Lambda_{2}\left(\omega\left(-2_{\mathrm{L}} X\right)\right) \rightarrow \Lambda_{2}(\omega) \text {. }
$$

This map vanishes twice on ${ }_{\mathrm{L}} D$, hence induces

$$
\Lambda_{2}\left(\omega\left(-2_{\mathrm{L}} X\right)\right)\left(2_{\mathrm{L}} D\right) \rightarrow \Lambda_{2}(\omega),
$$

which again is an isomorphism over over the interior, ${ }_{\mathrm{L}} D^{o}$ (indeed over this interior a local equation for the boundary divisor $\partial$ pulls back to a local equation for ${ }_{\mathrm{L}} X$ in $X$ and a local equation for ${ }_{\mathrm{L}} D$ in $X_{B}^{[2]}$ ). Then we get a (left) comparison diagram, which is a commutative square

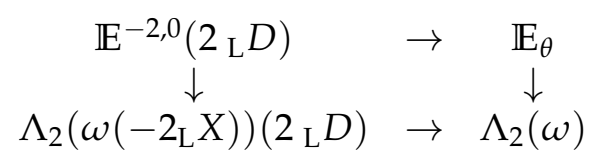

in which the left column is just the ordinary Brill-Noether map associated to $\omega\left(-2{ }_{\mathrm{L}} X\right)$; of course, there are analogous diagrams, one with left column replaced by

$$
\mathbb{E}^{-1,0}\left({ }_{\mathrm{L}} D\right) \rightarrow \Lambda_{2}\left(\omega\left({ }_{\mathrm{L}} X\right)\right)\left({ }_{\mathrm{L}} D\right),
$$

and two more with left replaced by right. So at least over ${ }_{\mathrm{L}} D^{o}, \phi_{\theta}$ can be identified with the Brill-Noether map associated to $\omega\left(-_{\mathrm{L}} X\right)$ and its restriction on ${ }_{\mathrm{L}} X$. That restriction coincides with the complete linear system $\omega_{\mathrm{L} X}(3 \theta)$ if $\left({ }_{\mathrm{R}} X,{ }_{\mathrm{R}} \theta\right)$ is non-hyperelliptic (so that ${ }_{\mathrm{R}} \theta$ is not a base point of $\left.\omega_{\mathrm{R}} X\left(-{ }_{\mathrm{R}} \theta\right)\right)$; otherwise, the restriction of $\omega\left(-2_{\mathrm{L}} X\right)$ on ${ }_{\mathrm{L}} X$ is $\left|\omega_{\mathrm{L} X}\left({ }_{\mathrm{L}} \theta\right)\right|+{ }_{\mathrm{L}} \theta$, i.e. has an imposed base point. Note that the twist involved coincides with that coming from the sepcanonical enlargement of the canonical system on $X$. On the other hand, it is elementary that a pair of distinct smooth points, each on one of ${ }_{\mathrm{L}} X,{ }_{\mathrm{R}} X$ are separated by the canonical system. Thus we conclude

Lemma 3.2. Notations as above, let $z \in X_{B}^{[2]}$ be disjoint from $\theta$. Then

(i) if $z$ is contained in one side of $\theta$, the image of the modified Brill- Noether map $\phi_{\theta}$ coincides over $z$ with that of the Brill-Noether map associated to the sepcanonical system;

(ii) if $z$ meets both sides of $\theta, \phi_{\theta}$ has maximal rank over $z$.

In this sense, the modified Brill-Noether realizes the goal of reviving dead sections. The case of schemes not disjoint from $\theta$ will follow from the calculations of the next subsection.

It is worth noting at this point that the normal bundle of ${ }_{\mathrm{L}} D$, which features in the intersection theory of the modifications $\mathbb{E}_{\theta}$, is easy to calculate. First recall that by Faber's result in [5], the 
conormal bundle to $\partial_{\theta} \rightarrow B$ is $\psi_{\theta}={ }_{L} \psi_{\theta} \otimes_{R} \psi_{\theta}$, i.e. the tensor product of the branch cotangents, and it follows easily that

$$
\check{N}_{\mathrm{L} X(\theta) / X}=\mathcal{O}\left({ }_{\mathrm{L}} \theta\right) \otimes \psi_{\theta}
$$

(compare the proof of Lemma 3.3). This can easily be extended to the Hilbert scheme. The formula uses the operation $[2]_{*}$ discussed in $\$ 4.3$.

Lemma 3.3. The conormal bundle of ${ }_{\mathrm{L}} D={ }_{\mathrm{L}} X(\theta){ }_{\partial_{\theta}}^{[2]}$ in $X_{B}^{[2]}$ is $[2]_{*}\left({ }_{\mathrm{L}} \theta\right) \otimes_{\mathrm{L}} \psi \otimes{ }_{\mathrm{R}} \psi$, where ${ }_{\mathrm{L}} \psi=$ $\left({ }_{L} \theta\right)^{*}\left(\omega_{L} X / \partial_{\theta}\right)$, considered as a line bundle on $\partial_{\theta}$ via viewting ${ }_{L} \theta$ as a cross-section ${ }_{L} \theta: \partial_{\theta} \rightarrow{ }_{L} X / \partial_{\theta}$, and likewise ${ }_{\mathrm{R}} \psi$.

Proof. By Faber's result [5], the conormal bundle of $\partial_{\theta}$ in $B$ is $\psi_{\theta}$. Hence there is an injection $\psi_{\theta} \rightarrow \mathcal{O}_{L} D\left(-{ }_{L} D\right)$ which, because $X_{\partial_{\theta}}^{[2]}$ has normal crossings, vanishes simply on $[2]_{*}\left({ }_{L} \theta\right)$, which is the intersection of ${ }_{\mathrm{L}} D$ with the other components. This proves our assertion.

For convenience, we will denote $[2]_{*}\left({ }_{L} \theta\right)$ by $\left({ }_{L} \theta\right)^{[2]}$. We will also need to compute selfintersections of $\left.{ }_{\mathrm{L}} \theta\right)^{[2]}$ within ${ }_{\mathrm{L}} D$. This follows from the following

Lemma 3.4. Let $X / B$ be a family of curves with a section $\theta$ contained in the smooth part. Let $\theta^{[2]} \subset X_{B}^{[2]}$ denote the locus of schemes meeting $\theta$ and $S_{\theta}$ the locus of schemes equal to $2 \theta$ and $\psi_{\theta}=\omega_{X / B} . \theta$. Then

$$
\left(\theta^{[2]}\right)^{i} \sim S_{\theta} \cdot\left(-2 \psi_{\theta}\right)^{i-1}, i \geq 1 .
$$

Proof. The case $i=1$ is clear. For $i=2$, we need to compute the normal bundle of $\theta^{[2]}$ restricted on $S_{\theta}$. In coordinates, a point in $S_{\theta}$ is represented by a scheme $x^{2}$ and its neighborhood by a deformation of the form $x^{2}+a x+b$. The equation of $\theta^{[2]}$ is $b$, which may be identified with $\omega_{X / B} \cdot \theta$ via $(x+\eta)^{2}=x+2 \eta x+\eta^{2}$. This shows the case $i=2$, and the case of higher $i$ follows.

3.3. Local forms. We analyze $\phi_{\theta}$ locally. We work near a fibre

$$
X_{0}=\left({ }_{\mathrm{L}} X(0),{ }_{\mathrm{L}} \theta(0)\right) \cup\left({ }_{\mathrm{R}} X(0),{ }_{\mathrm{L}} \theta(0)\right)
$$

comprised of 2 hyperelliptic pairs, as other cases are simpler. To begin with, using the results of $\$ 1.2$ on normal forms, we have adapted bases for the Hodge bundles $\mathbb{E}_{* X}$ so that the evaluation maps $\mathbb{E}_{*} \rightarrow_{*} \omega$ take the form

$$
\begin{array}{r}
\left(1, x^{2}+z_{1} x, x^{4}+z_{2} x^{3}, \ldots, x^{2 g_{1}-2}+z_{g_{1}-1} x^{2 g_{1}-3}\right), \\
\left(1, y^{2}+w_{1} y, y^{4}+w_{2} y^{3}, \ldots, y^{2 g_{2}-2}+w_{g_{2}-1} y^{2 g_{2}-3}\right)
\end{array}
$$

such that the locus of hyperelliptic pairs $\left({ }_{*} X, * \theta\right)$ is defined by $z_{1}=\ldots=z_{g_{1}-1}=0$ or $w_{1}=\ldots=$ $w_{g_{2}-1}=0$. Then the evaluation map $\mathbb{E} \rightarrow \omega$ takes the form (where we have written the $Y$ basis negatively, left of the bar)

$$
\left(\ldots, y^{5}+w_{2} y^{2}, y^{3}+w_{1} y^{2}, y \mid x, x^{3}+z_{1} x^{2}, x^{5}+z_{2} x^{2}, \ldots\right)
$$

Next, we work on the Hilbert scheme, locally near the locus of schemes supported on $\theta$; the case of the rest of the boundary of ${ }_{\mathrm{L}} D$ (i.e. ${ }_{\mathrm{L}} X \times{ }_{\mathrm{R}} \theta$ ) is similar and simpler. In terms of the coordinates $u=y_{2} / x_{1}$ etc. above $(\$ 2)$, we will analyze $\mathbb{E}_{\theta}$ over the open set where $u$ is regular, 
the other case, where $v=1 / u$ is regular, being similar. Set $\sigma_{i}=\sigma_{i}^{x}$. We will use the rules (2.0.2). We factor the Brill-Noether $\phi$ through $E_{\theta}$ :

$$
\left(\pi^{[2]}\right)^{*}(\mathbb{E}) \rightarrow \mathbb{E}_{\theta} \stackrel{\phi_{\theta}}{\rightarrow} \Lambda_{2}(\omega) .
$$

In the local coordinates above, factoring through $\mathbb{E}_{\theta}$ means the matrix for the factored mapping $\phi_{\theta}$ has $u$ factored out of the -1 column and $u^{2}$ factored out of the farther negative columns, i.e.

$$
\left[\begin{array}{ccc|ccc}
\ldots & u\left(\sigma_{1}^{3}-2 \sigma_{1} \sigma_{2}\right)+w_{1}\left(\sigma_{1}^{2}-\sigma_{2}\right) & \sigma_{1} & 0 & -\sigma_{2} \sigma_{1}-z_{1} \sigma_{2} & \ldots \\
\ldots & u\left(\sigma_{2}-\sigma_{1}^{2}\right)-w_{1} \sigma_{1} & -1 & 1 & \sigma_{1}^{2}-\sigma_{2}+z_{1} \sigma_{1} & \ldots
\end{array}\right]
$$

Here the matrix for $\left(\pi^{[2]}\right)^{*}(\mathbb{E}) \rightarrow \Lambda_{2}(\omega)$ itself has the same right side as the above, and left side equal to the $y$-mirror of the right side. By applying suitable column operations, i.e. composing with an automorphism of $E_{\theta}$, which does not affect degeneracy, we can kill off all multiples of $\sigma_{1}$ in the top row, except in column -1 , then kill off the entire second row except in column +1 , ending up with

$$
\Lambda_{\theta}=\left[\begin{array}{ccc|ccc}
\cdots & -w_{1} \sigma_{2} & \sigma_{1} & 0 & -z_{1} \sigma_{2} & \cdots \\
\cdots & 0 & 0 & 1 & 0 & \ldots
\end{array}\right]
$$

Now the ideal of $2 \times 2$ minors of $\Lambda_{\theta}$ is

$$
I_{2}\left(\Lambda_{\theta}\right)=\left(\sigma_{1}, z_{1} \sigma_{2}, \ldots, z_{g_{1}-1} \sigma_{2}, w_{1} \sigma_{2}, \ldots, w_{g_{2}-1} \sigma_{2}\right)
$$

and note that $u \sigma_{2}=u x_{1} x_{2}=y_{2} x_{2}=t$ is the equation of the boundary, which reflects the fact that in the open set we are considering (the one where $u$ is regular), the boundary is the union of ${ }_{\mathrm{L}} D=\left({ }_{\mathrm{L}} X\right)_{\delta}^{[2]}$, with equation $u$, and ${ }_{\mathrm{L}} X \times{ }_{\mathrm{R}} X$, with equation $\sigma_{2}$. Also, $w_{1}, \ldots, w_{g_{2}-1}$ define the locus in $\delta$ where $\left({ }_{\mathrm{R}} X, \mathrm{R} \theta\right)$ is a hyperelliptic pair.

For future reference, it is important to note that the above normal form (3.3.5) depends only on the normal form (3.3.2) for the (1-point) evaluation map.

Now set

$$
R_{\theta}=\operatorname{Zeros}\left(\sigma_{1}, \sigma_{2}\right) .
$$

This is the inverse image of the cycle $2 \theta$, a cross-section of $X_{\delta}^{(2)}$. In fact, $R_{\theta}$ is a $\mathbb{P}^{1}$-bundle over $\delta$, called the node scroll associated to $\theta$. Also let

$$
T=\operatorname{Zeros}\left(\sigma_{1}, z_{1}, \ldots, z_{g_{1}-1}, w_{1}, \ldots, w_{g_{2}-1}\right)
$$

This is a regular subscheme of codimension $g-1$, which coincides with $\mathcal{H} \mathcal{E}_{g}^{2}$ over the interior $B^{o} \subset B$ i.e. the set of smooth curves, and whose boundary portion is the locus $\mathcal{H E}_{g_{1}, g_{2}}^{2}$ of pairs of pointed hyperelliptics, which sits in a Cartesian diagram

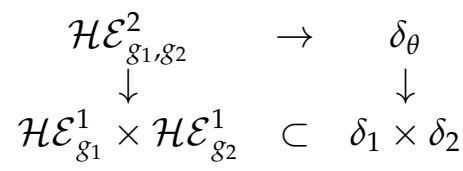

Now by (3.3.6), the degeneracy scheme $\mathbb{D}_{2}\left(\phi_{\theta}\right)$ splits schematically

$$
\mathbb{D}_{2}\left(\phi_{\theta}\right)=T \cup R_{\theta} .
$$


Now because $\mathcal{H E}_{g_{1}, g_{2}}^{2}$ is of codimension $g-1$ in the boundary, while $T$ is a priori purely of codimension at most $g-1$ overall, i.e. in $X_{B}^{[2]}$, it follows that $T$ must be contained in, hence coincide with, the closure of the degeneracy locus of $\phi=\phi_{\theta}$ in $B^{o}$, i.e.

$$
T=\overline{\mathcal{H} \mathcal{E}_{B^{o}}^{2}} \text {. }
$$

Furthermore, $T$ is transverse to the diagonal, which in the current local coordinates has equation $\sigma_{1}^{2}-4 \sigma_{2}$. Therefore, $T$ meets the diagonal, which is isomorphic to the blowup of $X$ in $\theta$, with exceptional divisor $R_{\theta}$, in a regular subscheme of codimension $g-1$ of the diagonal.

Note we have shown all this near the 'finite' part of $R_{\theta}$, where $u$ is regular. By symmetry, it holds near the part where $v=1 / u$ is regular, hence near all of $R_{\theta}$. Also, we note that all the above assertions are true and much easier to verify off $R_{\theta}$. We conclude

Proposition 3.5. Given a separating node $\theta$ of $X / B$ and the associated modification $\phi_{\theta}$ of the BrillNoether map, we have

(i) The rank of $\phi_{\theta}$ is at least 1 everywhere.

(ii) The degeneracy locus $\mathbb{D}_{2}\left(\phi_{\theta}\right)$ splits schematically as

$$
R_{\theta} \cup \overline{\mathcal{H} \mathcal{E}_{B^{o}}^{2}}
$$

with $\overline{\mathcal{H} \mathcal{E}_{B^{\circ}}^{2}}$ regular of codimension $g-1$.

(iii) The boundary is a transverse intersection:

$$
\overline{\mathcal{H} \mathcal{E}_{B^{o}}^{2}} \cap \partial_{\theta}=\mathcal{H E}_{g_{1}, g_{2}}^{2}
$$

(iv) The 1-point hyperelliptic locus $\overline{\mathcal{H E}_{B^{\circ}}^{1}}$ is regular of codimension $g-1$ in the diagonal of $X_{B}^{[2]}$.

(v) The intersection

$$
R_{\theta} \cap \overline{\mathcal{H} \mathcal{E}_{B^{o}}^{2}}=\left.R_{\theta}\right|_{\mathcal{H E}_{g_{1}}^{1} \times \mathcal{H} \mathcal{E}_{g_{2}}^{1}} \simeq \mathbb{P}_{\mathcal{H E}_{g_{1}}^{1} \times \mathcal{H} \mathcal{E}_{g_{2}}^{1}}\left(\psi_{1} \oplus \psi_{2}\right) .
$$

Corollary 3.6. A length-2 subscheme $\tau$ of $X_{1} \cup_{\theta} X_{2}$ is a limit of a pair in the hyperelliptic involution of a smooth curve if and only if $X_{1}, X_{2}$ are hyperelliptic and either $\tau$ is in the hyperelliptic involution on $X_{1}$ or $X_{2}$, or $\tau$ is supported on $\theta$.

As promised, the above analysis also allows us to strengthen slightly the result of Lemma 3.2

Corollary 3.7. Suppose $z \in X_{B}^{[2]}$ is contained in one side of $\theta$ but is not supported on $\theta$. Then the image of the modified Brill- Noether map $\phi_{\theta}$ coincides over $z$ with that of the Brill-Noether map associated to the sepcanonical system.

For application in inductive arguments, we mention another consequence of the above computations, to the behavior of $\phi_{\theta}$ away from $\theta$ :

Corollary 3.8. For $X_{0}$ as above, if $p \in{ }_{\mathrm{L}} X(\theta)$ is a Weierstrass point distinct from ${ }_{\mathrm{L}} \theta$, then a local matrix for the modified Brill-Noether map $\phi_{\theta}$ near $2 p$ has the form (1.2.8) where $z_{2}, \ldots, z_{g}$ are local equations for the closure of the hyperelliptic locus near $0 \in B$. 
3.4. Case of multiples seps. We now extend the previous results to the case of multiple separating nodes. Thus let $\Theta=\left(\theta_{1}, \ldots, \theta_{n}\right)$ be a collection of separating nodes of the versal family $X / B$. Then we get a collection of mutually transverse echelon data as in (3.1.3):

$$
\chi(\Theta)=\left({ }_{\mathrm{L}} \chi\left(\theta_{i}\right),{ }_{\mathrm{R}} \chi\left(\theta_{i}\right), i=1, \ldots, n\right) .
$$

Let $\mathbb{E}_{\Theta}$ be the associated multi-echelon modification and

$$
\phi_{\Theta}: \mathbb{E}_{\Theta} \rightarrow \Lambda_{2}(\omega)
$$

the resulting modification of the Brill-Noether map.

Proposition 3.9. Notations as above. Then for any length-2 subscheme $z$ contained in a $\Theta$-separation component of a fibre $X_{0}$ but not supported on $\Theta$, the image of $\phi_{\Theta}$ over $z$ coincides with that of the BrillNoether map of the $\Theta$-sepcanonical system of $X_{0}$. Further, the degeneracy locus of $\phi_{\Theta}$ coincides near $\left(X_{0}\right)^{[2]}$ with

$$
\overline{\mathcal{H} \mathcal{E}^{2}} \cup \bigcup_{i=1}^{n} R_{\theta_{i}} .
$$

Proof. $Y$ be a $\Theta$-separation component of $X_{0}$ and assume as usual that each $\theta \in \Theta$ is oriented with $Y$ on its left $(Y$ may or may not meet $\theta)$. Set

$$
\mathbb{E}^{\Theta, Y}=\pi_{*}\left(\omega_{X / B}\left(-2 \sum_{i=1}^{n}{ }_{L} X\left(\theta_{i}\right)\right)\right) .
$$

Then we get a multi-sep comparison diagram:

$$
\begin{array}{ccc}
\mathbb{E}^{\Theta, Y}\left(\sum_{\mathrm{L}} D\left(\theta_{i}\right)\right) & \rightarrow & \mathbb{E}_{\Theta} \\
\downarrow & & \downarrow \phi_{\Theta} \\
\Lambda_{2}\left(\omega\left(-2 \sum_{\mathrm{L}} X\left(\theta_{i}\right)\right)\right)\left(2 \sum_{\mathrm{L}} D\left(\theta_{i}\right)\right) & \rightarrow & \Lambda_{2}(\omega) .
\end{array}
$$

By [18], Proposition 6.10, the left vertical map coincides with the ordinary Brill-Noether map associated to the $\Theta$-sepcanonical of $X$ restricted on $Y$. Moreover, for any scheme $z$ on $Y$ disjoint from $\Theta$, the lower horizontal map is an isomorphism. This proves the Proposition for all schemes $z$ on $Y$ disjoint from $\Theta$. The analysis of the diagram at a scheme meeting $\Theta$ is analogous to the case of a single sep in 3.3 . This concludes the proof.

A nodal curve $X_{0}$ is said to be of pseudo-compact type if it has no proper biseps; or equivalently, if every connected component of the separation of all seps of $X_{0}$ is 2-inseparable.

Corollary 3.10. If $X_{0}$ is a fibre of pseudo-compact type and $\Theta$ is the set of all seps occurring on $X_{0}$, , then near $X_{0}^{[2]}$, the degeneracy locus of $\phi_{\Theta}$ coincides schematically with

$$
\overline{\mathcal{H} \mathcal{E}^{2}} \cup \bigcup_{i=1}^{n} R_{\theta_{i}} .
$$

Proof. We will use Theorem 7.2 of [18] and argue as in $\$ 3.2$. To begin with, if $X_{0}$ is not hyperelliptic, then the fact that the sepcanonical system is essentially very ample, together with a local analysis similar to $\$ 3.3$ shows that the degeneracy locus of $\phi_{\Theta}$ locally equals $\cup R_{\theta_{i}}$. Therefore we may assume $X_{0}$ is hyperelliptic. Pick $\theta \in \Theta$, which is necessarily (bilaterally) hyperelliptic. Then the sepcanonical system of ${ }_{\mathrm{L}} X_{0}(\theta)$ is (simply) ramified on ${ }_{\mathrm{L}} \theta$. By induction, as in Corollary 3.8, we may assume $\mathcal{H E}^{1}{ }_{\mathrm{L} g} \times{ }_{\mathrm{R}} \partial$ is regular of codimension ${ }_{\mathrm{L}} g-1=g\left({ }_{\mathrm{L}} X_{0}(\theta)\right)-1$ in an 
appropriate versal family ${ }_{L} \partial \times_{R} \partial$ mapped to by $\partial_{\theta}$. Likewise for the right side. Therefore, the local analysis as in 3.2 applies, yielding the conclusion.

\section{MOdifying BRILl-NOETHER, CASE (II): DISJOINT SEPARATING BINODES}

Our next focus is on biseps, i.e. separating binodes. That this requires a modification of the Brill-Noether map is already clear from the fact that, for any smooth connected curve $C$ with points $p \neq q$, the system $\left|\omega_{C}(p+q)\right|$ fails to separate $p$ and $q$. Additionally, there are curves with a binode that is hyperelliptic on one side only, for which Brill-Noether drops rank even though they are not limits of hyperelliptics. Because this situation occurs in codimension 2, some birational modification of Hilb will be required as well before an echelon modification can be applied to the Hodge bundle. We begin in $\$ 4$ by analyzing a 'naive' approach to this issue, based on modifying the family of curves. The approach we will actually use, based on modifying the Hilbert scheme directly, will be considered subsequently, starting in $\$ 4.2$.

4.1. Azimuthal curves : Single binode. Fix a bisep, i.e. a properly separating binode $\underline{\theta}$ on our family $X / B$, with corresponding locus

$$
\partial=\partial_{\underline{\theta}} \subset B
$$

and sides ${ }_{\mathrm{L}} X,{ }_{\mathrm{R}} X$ defined over $\partial$. We view $\underline{\theta}$ as oriented, i.e. with fixed choice of sides. Typically, $\underline{\theta}$ will be defined only over a suitable analytic or étale neighborhood of a boundary curve. Our purpose in this subsection is to construct a modification of $X / B$, a new family called an azimuthal modification, in which ${ }_{\mathrm{L}} X$, at least, becomes a Cartier divisor. One could then look at the Hilbert scheme of the new family. In the sequel, we will actually do something a little different, viz. modify the Hilbert scheme directly. Nonetheless, comparing the two constructions will be important in analyzing and interpreting the latter one.

The idea of the construction is an obvious one: first blow up $\partial_{\underline{\theta}}$, then blow up the inverse image of ${ }_{\mathrm{L}} X$, which is a Weil divisor. We proceed with the details.

First, let $B(\underline{\theta})$ be the blowup of $B$ in $\partial_{\underline{\theta}}$, with exceptional divisor

$$
P(\underline{\theta})=\mathbb{P}\left(\psi_{1} \oplus \psi_{2}\right), \psi_{i}=_{L} \psi_{i} \otimes_{R} \psi_{i},
$$

and let $X_{B(\underline{\theta})}=X \times{ }_{B} B(\underline{\theta})$ be the base-changed family. Let $S_{i} \subset P(\underline{\theta})$ denote the section $\mathbb{P}\left(\psi_{i}\right)$. We recall from Definition 6.1 of [18] that elements of $P(\underline{\theta})$ are called middle azimuths at $\underline{\theta}$, irregular or regular according as they are in $S_{1} \cup S_{2}$ or not.

In local coordinates $x_{i} y_{i}=t_{i}$ near $\theta_{i}, i=1,2$, a local coordinate on a suitable open in $B(\underline{\theta})$ containing $P(\underline{\theta}) \backslash S_{1}$ is $w=\left[t_{2} / t_{1}\right]$ and there, the base-changed family is given by

$$
x_{1} y_{1}=t_{1}, x_{2} y_{2}=t_{1} w
$$

The inverse image of ${ }_{L} X$ here is defined near $\theta_{1}$ resp. $\theta_{2}$ by $y_{1}$ resp. $\left(y_{2}, t_{1}\right)$, so it is a Weil divisor, non Q-Cartier near $\theta_{2}$; similarly for ${ }_{\mathrm{R}} X$.

Now let

$$
b: X_{B(\underline{\theta}), L} \rightarrow X_{B(\underline{\theta})}
$$


denote the blowup of $X_{B(\underline{\theta})}$ along the Weil divisor ${ }_{L} X$. The family $X_{B(\underline{\theta}), L} / B(\underline{\theta})$ is called the left azimuthal modification of $X / B$ corresponding to the binode $\underline{\theta}$. The construction may be diagrammed as follows

$$
\begin{array}{rlccc}
X_{B(\underline{\theta}), L} & \rightarrow & X_{B(\underline{\theta})} & \rightarrow & X \\
& \searrow & \downarrow & \square & \downarrow \\
& B(\underline{\theta}) & \rightarrow & B
\end{array}
$$

with the upper left horizontal arrow a small blowup. Using local coordinates as above, especially (4.1.1), it follows that $X_{B(\underline{\theta}), L}$ is virtually smooth (smooth if $X / B$ is locally versal at $\underline{\theta}$ ).

Locally near the inverse image of $\theta_{2}$, where the function $w$ is regular, $X_{B(\underline{\theta}), L}$ is covered by opens $U_{1}, U_{2}$ so that $\left[t_{1} / y_{2}\right]$ (resp. $\left[y_{2} / t_{1}\right]$ ) is regular in $U_{1}$ (resp. $\left.U_{2}\right)$. There is a natural 'left side' divisor ${ }_{L} X(\underline{\theta})$, defined locally by $y_{2}$ in $U_{1}$, or $t_{1}$ in $U_{2}$, which coincides with the inverse image in the blowup of

$$
{ }_{\mathrm{L}} X=\left(t_{1}=y_{2}=0\right) .
$$

Recall that we are working over a neighborhood of $P(\underline{\theta}) \backslash S_{1}$ in $B(\underline{\theta})$. Over this neighborhood, the exceptional locus of the blowup is a $\mathbb{P}^{1}$ bundle $P_{\theta_{2}}$ over $\left.\underline{\theta}_{2}\right|_{S_{2}}$, which is of codimension 2 . Note $P_{\theta_{2}}$ has equations

$$
t_{1}=\left[t_{2} / t_{1}\right]=x_{2}=y_{2}=0 .
$$

In $U_{1}, P_{\theta_{2}}$ is defined by $y_{2},\left[t_{2} / t_{1}\right]$ (since

$$
\left.t_{1}=\left[t_{1} / y_{2}\right] y_{2}, x_{2}=\left[t_{1} / y_{2}\right]\left[t_{2} / t_{1}\right]\right) \text {. }
$$

Similarly in $U_{2}, P_{\theta_{2}}$ is defined by $t_{1}, x_{2}$. All in all, the exceptional locus of the blowup consists of $P_{\theta_{1}} \cup P_{\theta_{2}}$.

This construction is decidedly not left-right symmetric however. In $U_{1}$, the inverse image of ${ }_{\mathrm{R}} X$ is $\operatorname{Zeros}\left(t_{1}=\left[t_{1} / y_{2}\right] y_{2}, x_{2}=\left[t_{1} / y_{2}\right]\left[t_{2} / t_{1}\right]\right)$. Therefore it consists of the divisor of $\left[t_{1} / y_{2}\right]$, which is the proper transform ${ }_{\mathrm{R}} \tilde{X}$ of ${ }_{\mathrm{R}} X$, plus $P_{\theta_{2}}$. In $U_{2}$, the inverse image of ${ }_{\mathrm{R}} X$ coincides with $P_{\theta_{2}}$ and ${ }_{\mathrm{R}} \tilde{X}$ is disjoint from it. Thus, denoting the blowup map by $b$, we have all in all

$$
b^{-1}\left({ }_{\mathrm{R}} X\right)={ }_{\mathrm{R}} \tilde{X} \cup P_{\theta_{1}} \cup P_{\theta_{2}}
$$

where ${ }_{\mathrm{R}} \tilde{X}$ is a Cartier divisor mapping isomorphically to ${ }_{\mathrm{R}} X$. On the other hand,

$$
P_{\theta_{1}} \cup P_{\theta_{2}} \subset{ }_{\mathrm{L}} X(\underline{\theta})
$$

therefore ${ }_{\mathrm{L}} X(\underline{\theta})$, the inverse image of ${ }_{\mathrm{L}} X$, is a divisor. In fact, ${ }_{\mathrm{L}} X(\underline{\theta})$ is just the blowup of ${ }_{\mathrm{L}} X_{P(\underline{\theta})}$ in its regular codimension-2 subvariety $\left.\theta_{1}\right|_{S_{1}} \amalg \theta_{2} \mid S_{2}$ (this has codimension 3 in $X_{B(\underline{\theta})}$ ). In the above coordinates, $\theta_{2} \mid S_{2}$ is defined by $\left[t_{2} / t_{1}\right]=x_{2}=0$.

The full boundary of the azimuthal modification is the union:

$$
X_{L, B(\underline{\theta})} \times{ }_{B(\underline{\theta})} P(\underline{\theta})={ }_{\mathrm{L}} X(\underline{\theta}) \cup{ }_{\mathrm{R}} \tilde{X}
$$

and its fibres (with $\underline{\theta}$ given) are called left azimuthal curves, regular or irregular, or curves with a left azimuthal binode in $\underline{\theta}$.

Remarks 4.1. We list some elementary properties of this construction. 
1) A (left or right) regular azimuthal fibre is isomorphic to the corresponding fibre over $B$. In this case, the left and right azimuthal structures on $\underline{\theta}$ are the same, constant over the fibre, and simply given by a regular element (azimuth)

$$
\mathfrak{a} \in \mathbb{P}\left(\psi_{\theta_{1}} \oplus \psi_{\theta_{2}}\right)=\mathbb{P}\left(T_{\mathrm{L} X, \theta_{1}}^{*} \otimes T_{\mathrm{R} X, \theta_{1}}^{*} \oplus T_{\mathrm{L} X, \theta_{2}}^{*} \otimes T_{\mathrm{R} X, \theta_{2}}^{*}\right) .
$$

2) As for left irregular azimuthal fibres, living over $S_{1} \amalg S_{2}, S_{1}$ is the (transverse) intersection of $P(\underline{\theta})$ with the proper transform of $\partial_{\theta_{1}}$, the boundary divisor corresponding to $\theta_{1}$. It parametrizes semistable triangular curves of the form $Y={ }_{\mathrm{L}} X \cup{ }_{\mathrm{R}} X \cup F$ where $F$ is a bridge $\mathbb{P}^{1}$ meeting ${ }_{\mathrm{L}} X,{ }_{\mathrm{R}} X$ in ${ }_{\mathrm{L}} \theta_{1}, \mathrm{R}_{\mathrm{R}} \theta_{1}$, respectively and ${ }_{\mathrm{L}} X$ and ${ }_{\mathrm{R}} X$ meet additionally in $\theta_{2}$. Thus $Y$ is stably equivalent to ${ }_{\mathrm{L}} X \cup{ }_{\mathrm{R}} X$, the corresponding fibre of $X / B$. Note that by construction, $F \subset{ }_{\mathrm{L}} X(\underline{\theta})$ (' $F$ tilts left') (because we are in the left azimuthal modification). Going out of the point $[Y] \in S_{1}$ in the direction of $P(\underline{\theta})$ corresponds to smoothing the node ${ }_{L} \theta_{1}$, keeping the other two as nodes. Going out in the direction of $\partial_{\theta_{1}}$ does the opposite, smoothing ${ }_{\mathrm{R}} \theta_{1}$ and $\theta_{2}$ and keeping ${ }_{L} \theta_{1}$ as a node.

Obviously, analogous comments apply with 1 replaced by 2 and $L$ replaced by R. Thus, a bridge at either $\theta_{i}$ can tilt left or right.

3) It follows from (4.1.3) that the normal bundle to ${ }_{\mathrm{L}} X(\underline{\theta})$ is

$$
\check{N}_{\mathrm{L} X(\underline{\theta}) / X_{\mathrm{L}, B(\underline{\theta})}}=\pi^{*}\left(\mathcal{O}_{P(\underline{\theta})}(1)\right) \otimes \mathcal{O}\left({ }_{\mathrm{L}} \underline{\theta}^{\prime}\right)
$$

where ${ }_{\mathrm{L}} \underline{\theta^{\prime}}$ is the pullback of ${ }_{\mathrm{L}} \underline{\theta}$, which coincides with $\underline{\theta}$ over the regular azimuthal fibres; over the irregular ones, the node $\theta_{i}$ is replaced by the other node on $F$ (i.e. where $F$ meets ${ }_{\mathrm{R}} X$ ). Here the first factor corresponds to a choice of middle azimuth (see Definition 6.1 of [18]). We have

$$
\check{N}_{\mathrm{L}} X(\underline{\theta}) /\left.X_{\mathrm{L}, B(\underline{\theta})}\right|_{\mathrm{L}} \theta_{i}={ }_{\mathrm{R}} \psi_{i}
$$

In particular, for a regular azimuthal fibre, $\check{N}_{\mathrm{L}} X(\underline{\theta}) /\left.X_{\mathrm{L}, B(\underline{\theta})}\right|_{\underline{\theta}}$ parametrizes right azimuths at $\underline{\theta}$.

4) We refer to [18], $\S 6$ (especially Definition 6.1). A hyperelliptic binode $\underline{\theta}$ on a fibre $X_{0}$ admits a uniquely determined regular azimuthal structure $\zeta=\left({ }_{L} \zeta,{ }_{M} \zeta,{ }_{R} \bar{\zeta}\right)$ called a hyperelliptic azimuth. This results from the hyperelliptic identification between left (resp. right) cotangents at $\theta_{1}$ and $\theta_{2}$. Then $X_{0}$ corresponds to a unique regular azimuthal fibre on either the right or left azimuthal modifications.

On the other hand if a regular azimuthal fibre (left or right) $X_{{ }_{\mathrm{M}} \zeta}$ corresponds to a fibre $X_{0}$ of $X$ over $\partial_{\underline{\theta}}$ where $\underline{\theta}$ is a left- hyperelliptic binode, then $X_{{ }_{\mathrm{M}}} \zeta$ carries a canonical compatible azimuthal structure $\left({ }_{\mathrm{L}} \zeta,{ }_{\mathrm{M}} \zeta{ }_{\mathrm{R}} \zeta\right)$ where ${ }_{\mathrm{R}} \zeta$ is induced by ${ }_{\mathrm{L}} \zeta{ }_{\mathrm{M}} \zeta$ as in Definition 6.1 of [18].

5) The constructions and results of this subsection extend directly to a collection $\Theta=\left\{\underline{\theta}_{1}, \ldots, \underline{\theta}_{n}\right\}$ of disjoint biseps. This is because the boundary loci $\partial_{\underline{\theta}_{i}}$ are in general position so their common blowup $B(\Theta)$ is well behaved (smooth if $B$ is smooth and $X / B$ is versal). Also the Weil divisors ${ }_{\mathrm{L}} X\left(\underline{\theta}_{i}\right) \times{ }_{{\underline{\theta_{i}}}_{i}} B(\Theta)$ are in general position.

4.2. Modifying Hilb. In this section we construct azimuthal modifications applied directly on the Hilbert scheme. We continue with the notation and assumptions of the previous subsection. Consider the regular, codimension-2 subvarieties

$$
{ }_{\mathrm{L}} X_{\partial}^{[2]},{ }_{\mathrm{R}} X_{21}^{[2]} \subset X_{B}^{[2]}
$$


These meet transversely in the codimension- 4 subvariety $\underline{\theta}_{\partial}$ which is a cross-section over $\partial$. The transversality (even just properness) of the intersection easily implies that the ideal of the schematic union is a product

$$
\mathcal{J}_{\underline{\theta}}:=\mathcal{I}_{\left({ }_{\mathrm{L}} X\right)_{\partial}^{[2]} \cup\left({ }_{\mathrm{R}} X\right)_{\partial}^{[2]}}=\mathcal{I}_{\left({ }_{\mathrm{L}} X\right)_{\partial}^{[2]}} \mathcal{I}_{\left({ }_{\mathrm{R}} X\right)_{\partial}^{[2]}}
$$

and moreover that the successive blowup of $X_{B}^{[2]}$ in $\left({ }_{\mathrm{L}} X\right)_{\partial}^{[2]}$ and $\left({ }_{\mathrm{R}} X\right)_{\partial}^{[2]}$ in either order is isomorphic to the blowup of $X_{B}^{[2]}$ in $\left({ }_{L} X\right)_{\partial}^{[2]} \cup\left({ }_{\mathrm{R}} X\right)_{\partial}^{[2]}$ (see also the local calculations below).

We also consider the base-changed family $X_{B(\underline{\theta})}$. Let $P=P(\underline{\theta})$ and consider the Weil divisors

$$
\left({ }_{\mathrm{L}} X\right)_{P}^{[2]},\left({ }_{\mathrm{R}} X\right)_{P}^{[2]} \subset X_{B(\underline{\theta})}^{[2]}=X_{B}^{[2]} \times{ }_{B} B(\underline{\theta}) .
$$

These intersect in $\underline{\theta}_{P}$ itself, considered as relative length-2 subscheme of $X_{P} / P$, thus forming a section of $X_{P}^{[2]}$ and having codimension $3(!)$ in $X_{B(\underline{\theta})}^{[2]}$. We denote by $\mathcal{J}_{\underline{\theta}}^{\prime}$ the product ideal

$$
\mathcal{I}_{\left({ }_{\mathrm{L}} \mathrm{X}\right)_{P}^{[2]}} \cdot \mathcal{I}_{\left({ }_{\mathrm{R}} \mathrm{X}\right)}{ }_{P}^{[2]}
$$

i.e. the pullback ideal of $\mathcal{J}_{\underline{\theta}}$.

Definition 4.2. The azimuthal Hilbert scheme (resp. extended azimuthal Hilbert scheme) of $X / B$ associated to $\underline{\theta}$, denoted $X_{B}^{\{2\}}(\underline{\theta})$ (resp. $X_{B(\underline{\theta})}^{\{2\}}$ ) is the blowup of the ideal $\mathcal{J}_{\underline{\theta}}$ on $X_{B}^{[2]}$ (resp. $\mathcal{J}_{\underline{\theta}}^{\prime}$ on $\left.X_{B(\underline{\theta})}^{[2]}\right)$.

A point of $X_{B}^{\{2\}}(\underline{\theta})$ is called an azimuthal length-2 scheme of $X / B$ with respect to $\underline{\theta}$. We will see in Corollary 4.4 that in the case of an interior scheme (strictly to one side of $\underline{\theta}$ ), an azimuthal scheme in fact consists of a scheme together with a (left or right) azimuth at $\underline{\theta}$. We denote by ${ }_{\mathrm{L}} D(\underline{\theta}),{ }_{\mathrm{R}} D(\underline{\theta})$ the respective inverse images of $\left({ }_{\mathrm{L}} X(\underline{\theta})\right)_{P}^{[2]},\left({ }_{\mathrm{R}} X(\underline{\theta})\right)_{P}^{[2]}$ in $X_{B}^{\{2\}}(\underline{\theta})$.

Note the natural map

$$
X_{B(\underline{\theta})}^{\{2\}} \rightarrow X_{B}^{\{2\}}(\underline{\theta}) \times{ }_{B} B(\underline{\theta}) ;
$$

in fact $X_{B(\underline{\theta})}^{\{2\}}$ can be identified with the unique component of $X_{B}^{\{2\}}(\underline{\theta}) \times_{B} B(\underline{\theta})$ dominating $X_{B}^{\{2\}}(\underline{\theta})$. Clearly, $X_{B(\underline{\theta})}^{\{2\}}$ and $X_{B}^{\{2\}}$ are right-left symmetric, i.e. independent of the orientation of the binode $\underline{\theta}$. Though $X_{B(\underline{\theta})}^{\{2\}}$ is in some sense more natural (e.g. it is flat over $B(\underline{\theta})$ ), the objects we need to work with are defined already over its smaller relative $X_{B}^{\{2\}}(\underline{\theta})$, so the latter is ultimately preferable.

We describe $X_{B(\underline{\theta})}^{\{2\}}$ and $X_{B}^{\{2\}}(\underline{\theta})$ in local coordinates over the point $\underline{\theta}$; other local descriptions are simpler. We may assume the family is given in local analytic coordinates near $\theta_{1}, \theta_{2}$ respectively by

$$
x_{1} y_{1}=t_{1}, x_{2} y_{2}=t_{2}
$$

where $x_{1}, x_{2}$ and $y_{1}, y_{2}$ are respective local coordinates on open sets ${ }_{\mathrm{L}} X_{1},{ }_{\mathrm{L}} X_{2} \subset{ }_{\mathrm{L}} X,{ }_{\mathrm{R}} X_{1},{ }_{\mathrm{R}} X_{2} \subset$ ${ }_{\mathrm{R}} X$. Locally analytically near $\underline{\theta}, X_{B}^{[2]}$ is a Cartesian product $\left({ }_{\mathrm{L}} X_{1} \cup{ }_{\mathrm{R}} X_{1}\right) \times\left({ }_{\mathrm{L}} X_{2} \times{ }_{\mathrm{R}} X_{2}\right)$. Let $u=$ $u_{2} / u_{1}$ be an affine coordinate on the finite part of the projectivized normal bundle $\mathbb{P}\left(N_{\left(\mathrm{L}_{1},{ }_{\mathrm{L}} \theta_{2}\right) \mathrm{L}} X_{1} \times{ }_{\mathrm{L}} X_{2}\right)$, where $u_{1}, u_{2}$ are linear coordinates on the normal bundle (these will not be needed in the sequel). For later reference, the latter bundle can be identified as ${ }_{L} \psi_{1} \oplus_{L} \psi_{2}$ with the obvious notations. 
On the blowup $\mathcal{B} \ell_{\underline{\theta}} X_{B}^{[2]}$, we can write $u=x_{2} / x_{1}$ so $u$ measures the 'speed ratio' as 2 points approach the $y$-axis. Similarly for $v=v_{2} / v_{1}=y_{2} / y_{1}$ on the right side and $w=w_{2} / w_{1}=t_{2} / t_{1}$ on the base (i.e. $w$ is an affine coordinate on $P(\underline{\theta}) / \partial_{\underline{\theta}}$ ). So we can cover the exceptional locus in $X_{B(\underline{\theta})}^{\{2\}}$ by 8 opens where $\left(u\right.$ or $\left.u^{\prime}=u^{-1}\right)$ and $\left(v\right.$ or $\left.v^{\prime}=v^{-1}\right)$ and $\left(w\right.$ or $\left.w^{\prime}\right)$ are regular; in the case of $X_{B}^{\{2\}}(\underline{\theta})$, the $w^{\prime}$ s don't exist and 4 opens suffice. On the open where $u, v, w$ are regular, the equations for $X_{B(\underline{\theta})}^{\{2\}}$ are given by

$$
\begin{array}{r}
x_{1} y_{1}=t_{1}, x_{2}=u x_{1}, y_{2}=v y_{1}, t_{2}=w t_{1} \\
w=u v
\end{array}
$$

In the case of $X_{B}^{\{2\}}(\underline{\theta})$, the equations are

$$
\begin{array}{r}
x_{1} y_{1}=t_{1}, x_{2}=u x_{1}, y_{2}=v y_{1}, \\
t_{2}=x_{1} y_{1} u v
\end{array}
$$

In particular both are virtually smooth there, with local coordinates $x_{1}, y_{1}, u, v$ relatively over the base $(B$ or $B(\underline{\theta}))$. Here $x_{1}, y_{1}$ are equations for ${ }_{\mathrm{L}} D,{ }_{\mathrm{R}} D$ respectively and in the case of $X_{B(\underline{\theta})}^{\{2\}}$ these together make up the entire boundary component corresponding to $\underline{\theta}$, that is, $P=P(\underline{\theta})$ with equation $t_{1}=x_{1} y_{1}$. Analogous equations on the other covering opens show that $X_{B}^{\{2\}}(\underline{\theta})$ is smooth. For example, on the open where $u, v^{\prime}, w$ are regular, we have local coordinates $x_{1}, y_{2}, v^{\prime}, w$ and the boundary, with equation $t_{1}=x_{1} v^{\prime} y_{2}$, consists of ${ }_{\mathrm{L}} D$ with equation $y_{2},{ }_{\mathrm{R}} D$ with equation $x_{1}$, and ${ }_{\mathrm{L}} X \times{ }_{\mathrm{R}} X$, with equation $v^{\prime}$.

4.3. Normal bundles. Recall that in Lemma 3.3 we computed the conormal bundle to the divisor $\left({ }_{L} X(\theta)\right)_{B}^{[2]}$ in $X_{B}^{[2]}$, for a sep $\theta$. We now extend this to the case of a bisep and the associated azimuthal modification of the Hilbert scheme. For a line bundle $L$ on $X$, we denote by $[2]_{*} L$ or sometimes $L^{[2]}$ its 'norm', a line bundle on $X_{B}^{[2]}$ (cf. [17]). Succinctly,

$$
[2]_{*} L=L^{[2]}=\operatorname{det}\left(\Lambda_{2}(L)\right) \otimes \mathcal{O}\left(\Gamma^{(2)}\right)
$$

where $\Gamma^{(2)}$ is the discriminant divisor. Similarly we have $[2]_{*} \mathfrak{b}$ for a divisor $\mathfrak{b}$ on $X / B$ (for $\mathfrak{b}$ prime, $[2]_{*} \mathfrak{b}$ is geometrically the locus of schemes meeting $\left.\mathfrak{b}\right)$. Let $\underline{\theta}$ be a bisep, i.e. a properly separating oriented binode on a nodal curve $X$, with sides ${ }_{\mathrm{L}} X(\underline{\theta}),{ }_{\mathrm{R}} X(\underline{\theta})$, defined over a codimension-2 locus $\partial_{\underline{\theta}}$. Define the (linear) left azimuth bundle on $\left.{ }_{\mathrm{L}} X(\underline{\theta})\right)^{[2]}$ associated to this data as

$$
{ }_{\mathrm{L}} \tilde{\mathfrak{a}}_{X, \underline{\theta}}=[2]_{*}\left(\mathcal{O}\left({ }_{\mathrm{L}} \theta_{1}\right) \otimes \psi_{\theta_{1}}\right) \oplus[2]_{*}\left(\mathcal{O}\left({ }_{\mathrm{L}} \theta_{2}\right) \otimes \psi_{\theta_{2}}\right)
$$

Lemma 4.3. There is a canonical isomorphism

$$
{ }_{L} \tilde{\mathfrak{a}}_{X, \underline{\theta}} \simeq \check{N}_{(\mathrm{L} X(\underline{\theta}))_{\underline{\partial}}^{[2]} / X_{B}^{[2]}} .
$$

Proof. Indeed there is a canonical map

$$
\psi_{\theta_{1}} \oplus \psi_{\theta_{2}}=\check{N}_{\partial_{\underline{\theta}} / B} \rightarrow \check{N}_{(\mathrm{L} X(\underline{\theta}))_{\partial_{\underline{\theta}}}^{[2]} / X_{B}^{[2]}}=: \check{N} .
$$


The component map $\psi_{\theta_{i}} \rightarrow \Sigma \bar{N}$ drops rank precisely on the divisor $[2]_{*}\left({ }_{\mathrm{L}} \theta_{i}\right)$ so the saturation of the image of the component map is exactly $[2]_{*}\left(\mathcal{O}\left({ }_{L} \theta_{i}\right) \otimes \psi_{\theta_{i}}\right)$. Putting the two components together, we get a canonical map ${ }_{\mathrm{L}} \tilde{\mathfrak{a}}_{X, \theta} \rightarrow \tilde{N}$. This map is obviously an isomorphism in codimension 1, hence we obtain (4.3.2).

An element of the projectivization ${ }_{L^{a}}{ }_{X, \theta}:=\mathbb{P}\left({ }_{L} \tilde{\mathfrak{a}}_{X, \theta}\right)$ is called a left azimuthal scheme of length 2 (or left azimuthal structure on the underlying scheme). This projectivization coincides with the exceptional divisor of the blowup of $\left({ }_{L} X\right)_{B}^{[2]}$ on $X_{B}^{[2]}$. Note that it contains 2 distinguished sections, viz. $\mathbb{P}\left([2]_{*}\left(\mathcal{O}\left({ }_{L} \theta_{i}\right) \otimes \psi_{\theta_{i}}\right)\right), i=1,2$; elements of these are called singular azimuthal schemes, while elements of the complement of their union are said to be regular.

Note that the fibre of ${ }_{L} \tilde{a}_{X, \underline{\theta}}$ at ${ }_{L} \theta_{1}+{ }_{L} \theta_{2}$ is naturally isomorphic (by residue) to ${ }_{R} \psi_{\theta_{1}} \oplus_{\mathrm{R}} \psi_{\theta_{2}}$. On the other hand the fibre at any 'interior' scheme, i.e. one disjoint from ${ }_{\mathrm{L}} \theta_{1}+{ }_{\mathrm{L}} \theta_{2}$, is naturally isomorphic to $\psi_{\theta_{1}} \oplus \psi_{\theta_{2}}$. Thus

Corollary 4.4. Notations as above, an interior left [resp. left regular ] azimuthal scheme of $X$ with respect to $\underline{\theta}$ consists of an interior subscheme of ${ }_{\mathrm{L}} X(\underline{\theta})$ together with a middle [resp. middle regular] azimuth of $X$ at $\underline{\theta}$.

We need to work out the relevant normal bundles on the azimuthal Hilbert scheme $X_{B}^{\{2\}}(\underline{\theta})$. We denote by ${ }_{\mathrm{L}} D(\underline{\theta}),{ }_{\mathrm{R}} D(\underline{\theta})$, respectively, the projectivized normal bundles of $\left.{ }_{\mathrm{L}} X\right)^{[2]},\left({ }_{\mathrm{R}} X\right)^{[2]}$ in $X_{B}^{[2]}$, which coincide with the exceptional divisor of the respective blow up. By Lemma 4.3, we can identify

$$
{ }_{\mathrm{L}} D(\underline{\theta}) \simeq \mathbb{P}_{\left({ }_{\mathrm{L}} X\right)_{D}^{[2]}}\left({ }_{\mathrm{L}} \theta_{1}^{[2]} \otimes \psi_{\theta_{1}} \oplus{ }_{\mathrm{L}} \theta_{2}^{[2]} \otimes \psi_{\theta_{2}}\right)
$$

where we use the abbreviation $\theta^{[2]}$ for $[2]_{*} \theta$. So this is a split $\mathbb{P}^{1}$ bundle with a simple intersection theory. Ditto for the other side.

We denote by ${ }_{\mathrm{L}} D^{\dagger}(\underline{\theta}),{ }_{\mathrm{R}} D^{\dagger}(\underline{\theta})$ the respective inverse images of $\left.\left({ }_{\mathrm{L}} X\right)_{D}^{[2]},{ }_{\mathrm{R}} X\right)_{D}^{[2]}$ on $X_{B}^{\{2\}}$, both Cartier divisors and admitting a natural map, respectively, to ${ }_{*} D(\underline{\theta}), *=L, R$. Moreover, ${ }_{L} D^{\dagger}(\underline{\theta})$ is the projectivized normal bundle of the inverse image of $\left({ }_{\mathrm{L}} X\right)_{D}^{[2]}$ on the blowup of $\left({ }_{\mathrm{R}} X\right)_{D}^{[2]}$. This inverse image is just the blowup of $\left.{ }_{{ }_{L}} X\right)_{D}^{[2]}$ in the section ${ }_{L} \underline{\theta}={ }_{L} \theta_{1}+{ }_{L} \theta_{2}$. By transversality, the normal bundle of the inverse image is just the pullback of the normal bundle. Therefore,

$$
{ }_{\mathrm{L}} D^{\dagger}(\underline{\theta}) \simeq \mathbb{P}_{B_{\underline{\theta}}\left({ }_{\mathrm{L}} X\right)_{D}^{[2]}}\left({ }_{\mathrm{L}} \theta_{1}^{[2]} \otimes \psi_{\theta_{1}} \oplus{ }_{\mathrm{L}} \theta_{2}^{[2]} \otimes \psi_{\theta_{2}}\right)
$$

and therefore, with respect to this identification,

$$
\mathcal{O}_{{ }_{\mathrm{L}} D^{\dagger}(\underline{\theta})}\left(-{ }_{\mathrm{L}} D^{\dagger}(\underline{\theta})\right)=\mathcal{O}(1) .
$$

We call interior points of $X_{B}^{[2]}$, the points corresponding to schemes disjoint from $\underline{\theta}$. Similarly for spaces equipped with a map to $X_{B}^{[2]}$, such as its subschemes, e.g. ${ }_{\mathrm{R}} X^{[2]}$.

4.4. Modifying Brill-Noether. We will construct a modification of the Hodge bundle on the azimuthal Hilbert scheme $X_{B}^{\{2\}}(\underline{\theta})$. Essentially, there are two independent parts to this modification, for the left and right sides. 
Let $\mathbb{E}$ be the pullback of the Hodge bundle on the azimuthal Hilbert scheme $X_{B}^{\{2\}}(\underline{\theta})$. Over $\partial:=\partial_{\underline{\theta}}$, we have exact

$$
0 \rightarrow \pi_{*}\left(\omega_{\mathrm{R}} X\right) \rightarrow \mathbb{E}_{\partial_{\underline{\theta}}} \rightarrow \pi_{*}\left(\omega_{\mathrm{L}} X\left({ }_{\mathrm{L}} \theta_{1}+{ }_{\mathrm{L}} \theta_{2}\right)\right) \rightarrow 0 .
$$

Let ${ }_{\mathrm{L}} \mathbb{E},{ }_{\mathrm{L}} \mathbb{E}^{0}$ denote the respective pullbacks of $\pi_{*}\left(\omega_{\mathrm{L} X}\left({ }_{\mathrm{L}} \theta_{1}+{ }_{\mathrm{L}} \theta_{2}\right)\right), \pi_{*}\left(\omega_{\mathrm{L} X}\right.$ on ${ }_{\mathrm{L}} D^{\dagger}={ }_{\mathrm{L}} D^{\dagger}(\underline{\theta}) \subset$ $X_{B}^{\{2\}}(\underline{\theta})$, i.e. the pullback of ${ }_{\mathrm{L}} X_{\partial}^{[2]}$. Similarly for ${ }_{\mathrm{R}} \mathbb{E}$. Then over $X_{B}^{\{2\}}(\underline{\theta})$, we get exact

$$
\begin{aligned}
& 0 \rightarrow \mathbb{E}^{-1,0} \rightarrow \mathbb{E} \rightarrow{ }_{\mathrm{L}} \mathbb{E} \rightarrow 0 \\
& 0 \rightarrow \mathbb{E}^{0,-1} \rightarrow \mathbb{E} \rightarrow{ }_{\mathrm{R}} \mathbb{E} \rightarrow 0
\end{aligned}
$$

(these define $\mathbb{E}^{*}$ ), and also

$$
\begin{aligned}
& 0 \rightarrow{ }_{\mathrm{R}} \mathbb{E}^{0} \rightarrow \mathbb{E}_{\partial_{\underline{\theta}}} \rightarrow{ }_{\mathrm{L}} \mathbb{E} \rightarrow 0, \\
& 0 \rightarrow{ }_{\mathrm{L}} \mathbb{E}^{0} \rightarrow \mathbb{E}_{\partial_{\underline{\theta}}} \rightarrow{ }_{\mathrm{R}} \mathbb{E} \rightarrow 0 .
\end{aligned}
$$

Hence we get a (clearly transverse) pair of echelon data (with $D=\delta$ )

$$
{ }_{\mathrm{L}} \chi(\underline{\theta})=\left(\mathbb{E}^{-1,0}(\underline{\theta}),{ }_{\mathrm{L}} D^{\dagger}\right),{ }_{\mathrm{R}} \chi(\underline{\theta})=\left(\mathbb{E}^{0,-1}(\underline{\theta}), \mathrm{R}_{\mathrm{R}}^{\dagger}\right) .
$$

Note that the pullback of $\mathbb{E}^{-1,0}$ on $X_{B(\underline{\theta})}^{\{2\}}$ coincides with the pullback from $B(\underline{\theta})$ of $\pi_{*}\left(\omega\left(-{ }_{\mathrm{L}} X\right)\right)$. In this way this $\mathbb{E}^{-1,0}$ is similar to the analogously-denoted bundle in the separating node case, see $\$ 3$. These give rise to an echelon modification $\mathbb{E}_{\underline{\theta}}$, through which the Brill-Noether map factors yielding a map that we call the modified Brill-Noether map with respect to $\underline{\theta}$

$$
\phi_{\underline{\theta}}: \mathbb{E}_{\underline{\theta}} \rightarrow \Lambda_{2}(\omega) .
$$

Remark 4.5. Rather than take as our starting point the Hodge bundle itself, we could take its modification $\mathbb{E}_{\Theta}$ with respect to any collection of seps (see 3.4 ). More general cases will be considered below (e.g. 4 4.6).

Next we develop a convenient local normal matrix form for the Brill-Noether map and its modification $\phi_{\underline{\theta}}$ and derive an important dimension count for the degeneracy locus of $\phi_{\underline{\theta}}$. As $\phi_{\underline{\theta}}$ is a map of vector bundles, its degeneracy locus can be studied fibrewise, i.e. the fibre of the degeneracy locus over $0 \in B$ coincides with the degeneracy locus of the restriction of $\phi_{\underline{\theta}}$ over $X_{0}^{\{2\}}$ We work on a fixed fibre over $\partial_{\underline{\theta}}, X_{0}={ }_{\mathrm{L}} X \cup_{\mathrm{R}} X$. We will work near $\underline{\theta}_{0}:=\underline{\theta} \cap X_{0}$ and use the obvious local basis for $\Lambda_{2}$ with values $(1,0),(0,1)$ at $\left(\theta_{1}, \theta_{2}\right)$.

Case 1: $\underline{\theta}_{0}$ is (bilaterally) hyperelliptic on $X_{0}$.

Then for each $i>0$ there is a section $s_{-i}$ vanishing on ${ }_{\mathrm{R}} X$ and having local form $\left(x_{1}^{i}, x_{2}^{i}\right)$ on ${ }_{\mathrm{L}} X$ near $\left({ }_{L} \theta_{01},{ }_{L} \theta_{02}\right)$ (recall that $\left({ }_{L} X,{ }_{L} \theta_{01}+{ }_{L} \theta_{02}\right)$ is hyperelliptic). Similarly on the right, with local coordinates $y_{i}$. Then the Brill-Noether matrix takes the form

$$
\left[\begin{array}{lllllll}
\ldots & x_{1}^{2} & x_{1} & 1 & y_{1} & y_{1}^{2} & \ldots \\
\ldots & x_{2}^{2} & x_{2} & 1 & y_{2} & y_{2}^{2} & \ldots
\end{array}\right]
$$

where $X_{0}$ is locally defined by $x_{1} y_{1}=x_{2} y_{2}=0$, Now the azimuthal Hilbert scheme of $X_{0}$ is a $\mathbb{P}^{1} \times \mathbb{P}^{1}$-bundle over the Hilbert scheme, locally covered by 4 open affines $\mathbb{A}^{1} \times \mathbb{A}^{1}$ which may 
be labelled (finite, finite) etc. On the (finite, finite) part of the azimuthal Hilbert scheme $X_{0}^{\{2\}}(\underline{\theta})$, where $u, v$ are regular, the matrix $\Lambda_{\underline{\theta}}$ for $\phi_{\underline{\theta}}$ takes the form

$$
\left[\begin{array}{ccccccc}
\cdots & x_{1} & 1 & 1 & 1 & y_{1} & \cdots \\
\cdots & x_{1} u^{2} & u & 1 & v & y_{1} v^{2} & \cdots
\end{array}\right]
$$

which drops rank on the codimension-2 locus $u=v=1$. Note that this open set only meets ${ }_{\mathrm{L}} D^{\dagger}$, defined by $y_{1}$, and ${ }_{\mathrm{R}} D^{\dagger}$, defined by $x_{1}$; it misses the 'mixed' part of the (azimuthal) Hilbert scheme, and misses as well the locus of singular azimuths $u v=0$. In ${ }_{\mathrm{L}} D^{\dagger}, u=1$ defines the graph of the hyperelliptic involution while $v=1$ is the hyperelliptic right azimuth, i.e. the section of the $\mathbb{P}^{1}$-bundle parametrizing azimuths, corresponding to the hyperelliptic azimuth on ${ }_{\mathrm{R}} \underline{\theta}$. In ${ }_{\mathrm{L}} D^{\dagger}, v=1$ corresponds to the condition on sections of $\omega_{\mathrm{L}} X(\underline{\theta})(2 \mathrm{\theta} \underline{\theta})$ that they have the value at ${ }_{L} \theta$ dictated by the (left) hyperelliptic azimuthal condition. Similarly for ${ }_{R} D^{\dagger}$. The other open sets covering the azimuthal Hilbert scheme are handled similarly. We conclude that overall in this case, the fibre over 0 of the degeneracy locus of $\Lambda_{\underline{\theta}}$ has the form $x_{1} y_{1}=0$, i.e. is itself a nodal curve.

Case $2: \underline{\theta}_{0}$ not bilaterally hyperelliptic on $X_{0}$.

We may assume that $\left({ }_{\mathrm{R}} X, \mathrm{R}_{\mathrm{R}} \theta_{1}+{ }_{\mathrm{R}} \theta_{2}\right)$ is non- hyperelliptic as a pair. Then in the above calculation the basis on the right side can be taken of the form $\left(y_{1}, y_{2}^{2}\right),\left(y_{1}^{2}, y_{2}\right), \ldots$ and the corresponding modified Brill-Noether matrix will take the form

$$
\left[\begin{array}{ccccccc}
\cdots & x_{1} & 1 & 1 & 1 & y_{1} & \ldots \\
\ldots & x_{1} u^{2} & u & 1 & v^{2} y_{1} & v & \ldots
\end{array}\right]
$$

which has maximal rank near $y_{1}=0$. Off the locus $y_{1}=0$, the right half of the matrix is equivalent to the Brill-Noether matrix of ${ }_{\mathrm{R}} X$ itself, hence has maximal rank. Therefore, $\phi_{\underline{\theta}}$ has maximal rank everywhere.

We can summarize the foregoing discussion as follows.

Proposition 4.6. Let $\underline{\theta}$ be a properly separating binode of $X / B$ with associated boundary locus $\partial=\partial_{\underline{\theta}}$ and modified Brill-Noether map $\phi_{\underline{\theta}}$. Then for any fibre $X_{0}$ in the interior $\partial^{0}$ of $\partial$, i.e. having no seps or biseps off $\underline{\theta}$, the intersection $\mathbb{D}_{2}\left(\phi_{\underline{\theta}}\right) \cap X_{0}^{\{2\}}$ is 1-dimensional if $X_{0}$ is hyperelliptic and empty otherwise. When $X_{0}$ is hyperellptic, the intersection consists of the loci of hyperelliptic subschemes on each side of $\underline{\theta}$, endowed with the hyperelliptic azimuth at $\underline{\theta}$.

It follows from the Proposition that, assuming $X / B$ is versal, the codimension of $\mathbb{D}_{2}\left(\phi_{\underline{\theta}}\right) \cap$ $\pi^{-1}(\partial)$ in $B_{\underline{\theta}} X^{[2]}$ is at least

$$
(L g-1)+\left({ }_{R} g-1\right)+2+1=g
$$

(codimensions of hyperelliptic 2-pointed curve loci on left and right plus codimension of binodal locus plus fibre codimension). This number is greater than the expected codimension of the degeneracy locus $\mathbb{D}_{2}\left(\phi_{\underline{\theta}}\right)$ of $\phi_{\underline{\theta}}$, which equals $g-1$. Therefore this boundary locus cannot contribute a component to $\overline{\mathrm{D}}_{2}(\phi)$. Thus

Corollary 4.7. We have, schematically,

$$
\mathbb{D}_{2}\left(\phi_{\underline{\theta}}\right) \cap \pi^{-1}\left(B^{0} \cup \partial^{0}\right)=\overline{\mathbb{D}_{2}\left(\phi_{\underline{\theta}}\right) \cap \pi^{-1}\left(B^{0}\right)} \cap \pi^{-1}\left(B^{0} \cup \partial^{0}\right)=\overline{\mathbb{D}_{2}(\phi) \cap \pi^{-1}\left(B^{0}\right)} \cap \pi^{-1}\left(B^{0} \cup \partial^{0}\right)
$$


where $B^{0} \subset B$ is the interior, parametrizing smooth curves. In particular, every point of $\mathbb{D}_{2}\left(\phi_{\underline{\theta}}\right) \cap \pi^{-1}\left(\partial^{0}\right)$ is a specialization of a hyperelliptic divisor on a smooth hyperelliptic curve and conversely.

Remark 4.8. It is worth noting provisionally at this point that while the above construction took as its starting point the 'original' Hodge bundle and Brill-Noether map, we could just as well have started with the modifications $\mathbb{E}_{\Theta}, \phi_{\Theta}$, where $\Theta$ is the set of all seps of $X_{0}$, as in Proposition 3.9. More general results will be given below.

4.5. Interpretation. As in the case of a separating node (see 3.2), the azimuthal modification of Brill-Noether can be interpreted in terms of reviving sections of the canonical that vanish identically to one side of the binode. To do so, we must have an appropriate twisting divisor on the total space $X$, as the sides in question are defined in codimension 2 . This is accomplished by an azimuthal modification of the original family, as in 4.1 .

Thus, fixing notations as above, let $X_{B(\underline{\theta}), \mathrm{L}} / B(\underline{\theta})$ be the left azimuthal modification of $X / B$, which is a nodal family over $B(\underline{\theta})=\mathcal{B} \ell_{\partial_{\underline{\theta}}} B$, and is endowed with left and right boundary families ${ }_{\mathrm{L}} \tilde{X}_{P},{ }_{\mathrm{R}} \tilde{X}_{P}$ over the exceptional divisor $P=P(\underline{\theta}) \subset B(\underline{\theta})$, which as we recall parametrizes middle azimuths ${ }_{\mathrm{M}} \zeta$ at $\underline{\theta}$. Here the right boundary family projects isomorphically:

$$
{ }_{\mathrm{R}} \tilde{X}_{P} \simeq{ }_{\mathrm{R}} X_{P}={ }_{\mathrm{R}} X_{\partial} \times{ }_{\partial} P
$$

while the left one ${ }_{\mathrm{L}} \tilde{X}_{P}$ is the pullback of ${ }_{\mathrm{L}} X(\underline{\theta}) \subset X$ and isomorphic to the blowup of ${ }_{\mathrm{L}} X_{\partial} \times_{\partial} P$ in $\underline{\theta}_{P}=\underline{\theta} \times{ }_{\partial} P$. Over the complement of the locus of singular azimuths, i.e. the 2 distinguished sections of $P / \partial$, the modified family $X_{B(\underline{\theta}), \mathrm{L}}$ coincides with $X_{B(\underline{\theta})}$.

We consider the relative Hilbert scheme for this modified family, viz. $\left(X_{B(\underline{\theta}), \mathrm{L}}\right)_{B(\underline{\theta})}^{[2]}$ (note the outer $B(\underline{\theta})$ subscript indicates that this is the relative Hilbert scheme). This comes equipped with a map to the symmetric product $\left(X_{\mathrm{L}, B(\underline{\theta})}\right)_{B(\underline{\theta})}^{(2)}$, hence to $X_{B(\underline{\theta})}^{(2)}$. Therefore we get a correspondence diagram

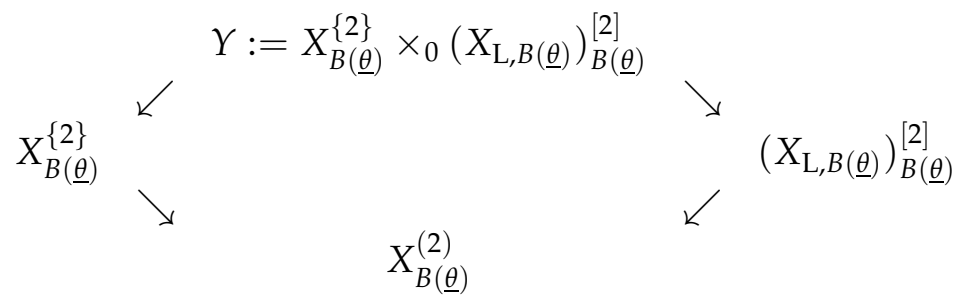

where $\times_{0}$ denotes the unique dominant component (over either factor) of the fibre product over $X_{B(\underline{\theta})}^{(2)}$. Now up on $Y$, we have all the sheaves we need to work as in (3.2.1). Thus, suppressing various pullbacks, we have a map

$$
\Lambda_{2}\left(\omega\left(-{ }_{\mathrm{L}} X_{P}\right)\right) \rightarrow \Lambda_{2}(\omega)
$$

which vanishes on ${ }_{\mathrm{L}} D^{\dagger}$, the inverse image of ${ }_{\mathrm{L}} X_{P}^{[2]}$, and we get, as in the case of separating nodes (see (3.2.1)), a comparison diagram (where we recall that $\mathbb{E}^{-1,0}$ may be identified as the pullback of 
$\pi_{*}\left(\omega\left(-{ }_{\mathrm{L}} X_{P}\right)\right)$ from $X \times_{B} B(\underline{\theta})$, hence also as the pullback of $\pi_{*}\left(\omega\left(-{ }_{\mathrm{L}} \tilde{X}_{P}\right)\right)$ from $\left.X_{B(\underline{\theta}) \mathrm{L}} / B(\underline{\theta})\right)$ :

$$
\begin{array}{ccc}
\mathbb{E}^{-1,0}\left({ }_{\mathrm{L}} D^{\dagger}\right) & \rightarrow & \mathbb{E}_{\underline{\theta}} \\
\downarrow & \cup & \downarrow \\
\Lambda_{2}\left(\omega\left({ }_{\mathrm{L}} X_{P}\right)\right)\left({ }_{\mathrm{L}} D^{\dagger}\right) & \rightarrow & \Lambda_{2}(\omega) .
\end{array}
$$

Here the horizontal maps are isomorphisms over the interior of ${ }_{\mathrm{L}} D^{+}$, i.e. the pullback of ${ }_{\mathrm{L}} X_{B}^{[2]} \backslash$ $[2]_{*}(\underline{\theta})$. Moreover, the right column, i.e. $\phi_{\underline{\theta}}$, is already defined over $X_{B}^{\{2\}}(\underline{\theta})$ and does not explicitly depend on a choice of middle azimuth. As for the left column, which does involve an explicit choice of middle azimuth, it follows from (4.1.5) that, at least over a fibre $X_{0}$ corresponding to a regular (middle) azimuth ${ }_{\mathrm{M}} \zeta, \omega\left(-_{\mathrm{L}} X_{0}\right)$ coincides with $\left(\omega_{\mathrm{L}} \mathrm{X}_{0}\left(2_{\mathrm{L}} \underline{\theta}\right) \otimes{ }_{\mathrm{M}} \zeta\right) \cup \omega_{\mathrm{R}} X_{0}$ (we note that, being regular, ${ }_{\mathrm{M}} \zeta$ is naturally isomorphic by projection to both $\psi_{1}$ and $\psi_{2}$ ) .

An interior point $z$ of ${ }_{L} D^{\dagger}$ consists of an underlying subscheme $z_{0}$ of ${ }_{L} X(\underline{\theta})$ plus a middle azimuth ${ }_{\mathrm{M}} \zeta$ at $\underline{\theta}$, and the latter determines a gluing of $\omega_{\mathrm{L}} \mathrm{X}_{0}\left(2_{\mathrm{L}} \underline{\theta}\right)$ and $\omega_{\mathrm{R}} X_{0}$ at $\theta_{1}$ and $\theta_{2}$, up to a common scalar. Then given a right azimuth ${ }_{\mathrm{R}} \zeta$ at $\underline{\theta}$, e.g. a hyperelliptic one, it determines a left azimuth ${ }_{\mathrm{L}} \zeta={ }_{\mathrm{M}} \zeta_{\mathrm{R}} \zeta^{-1}$ at $\underline{\theta}$ which varies with ${ }_{\mathrm{M}} \zeta$. If sections of $\omega_{\mathrm{R}} \mathrm{X}_{0}$ all satisfy the azimuthal condition ${ }_{R} \zeta$ (as in the hyperelliptic case), then as $z$ varies fixing the underlying scheme, the left column of (4.5.2) corresponds to subsystems of $\omega_{\mathrm{L}} X_{0}(\underline{\underline{\theta}})\left(2_{\mathrm{L}} \underline{\underline{\theta}}\right)$ determined by a varying azimuthal condition at $\mathrm{L}_{\mathrm{\theta}}$. Therefore, over regular azimuthal subschemes $z$ in the interior of ${ }_{\mathrm{L}} D^{\dagger}$, the image of the right column, i.e. of the modified Brill-Noether $\phi_{\underline{\theta}}$, coincides with that of the Brill-Noether map associated to the sepcanonical system $\left|\omega_{X_{0}}\right|{ }^{\text {sep }}$ restricted on ${ }_{L} X_{0}$, i.e. sections satisfying the residue condition (automatic in this case) and, when $\left({ }_{\mathrm{R}} X_{0}(\underline{\theta}),{ }_{\mathrm{R}} \underline{\theta}\right)$ is hyperelliptic, the azimuthal condition ${ }_{\mathrm{L}} \zeta={ }_{\mathrm{M}} \zeta_{\mathrm{R}} \zeta^{-1}$. By direct inspection, considering the matrix $\Lambda_{\underline{\theta}}$ above, the same also holds at the boundary of ${ }_{\mathrm{L}} D^{\dagger}$, i.e. the schemes meeting ${ }_{\mathrm{L}} \underline{\theta}$, and for the singular azimuths, i.e. those corresponding to replacing the twist $2{ }_{\mathrm{L}} \underline{\theta}$ by ${ }_{\mathrm{L}} \underline{\theta}+{ }_{\mathrm{L}} \theta_{i}, i=1$, 2 . Of course, $\phi_{\underline{\theta}}$ is left-right symmetric as well. Thus:

Lemma 4.9. For subschemes entirely on one side of $\underline{\theta}$, the image of the modified Brill-Noether map coincides with that of the Brill-Noether map associated to the $\underline{\theta}$-sepcanonical system of $X$.

Now consider a fibre $X_{0}$ in the 'interior' of $\partial_{\underline{\theta}}$, in the sense that $\left({ }_{L} X_{0}(\underline{\theta}),{ }_{L} \underline{\theta}\right)$ and $\left({ }_{R} X_{0}(\underline{\theta}),{ }_{R} \underline{\theta}\right)$ are 2-inseparable (see $\S 3$ of [18]); equivalently, $X_{0}$ has no other seps or biseps besides $\underline{\theta}$. If $\left({ }_{\mathrm{R}} X_{0}, \mathrm{R} \underline{\theta}\right)$ is not hyperelliptic, clearly $\left|\omega_{\mathrm{L}} X_{0}(\underline{\theta})\left(2_{\mathrm{L}} \underline{\theta}\right) \cup \omega_{\mathrm{R}} X_{0}\right|$ induces on the left side the complete linear system $\left|\omega_{L} X_{0}(\underline{\theta})\left(2_{L} \underline{\theta}\right)\right|$, necessarily very ample. Consequently, whenever $X_{0}$ is not hyperelliptic, $\phi_{\underline{\theta}}$ is surjective over $\left(X_{0}\right)^{\{2\}}$.

On the other hand if $X_{0}$ is hyperelliptic, a similar argument shows that $\phi_{\underline{\theta}}$ drops rank precisely on the hyperelliptic azimuthal schemes, i.e. those regular azimuthal schemes of the form $\left(z_{0},{ }_{\mathrm{R}} \zeta\right)$ where $z_{0}$ is a hyperelliptic divisor on ${ }_{L} X_{0}(\underline{\theta})$ and ${ }_{\mathrm{R}} \zeta$ is the hyperelliptic azimuth on ${ }_{\mathrm{R}} \underline{\theta}$, or the analogous schemes on the right. Note $\phi_{\underline{\theta}}$ has maximal rank at the singular azimuthal schemes as well as the mixed schemes (those on both sides of $\underline{\theta}$ ). Thus, if we let $\mathcal{H E}_{B}^{\{2\}} \subset X_{B}^{\{2\}}$ denote the locus of hyperelliptic azimuthal schemes, we conclude:

Corollary 4.10. Over the fibers having no sep or bisep besides $\underline{\theta}$ the degeneracy locus of $\phi_{\underline{\theta}}$ is equal to $\mathcal{H E}_{B}^{\{2\}}$ and coincides with the closure of the smooth hyperelliptic locus $\mathcal{H} \mathcal{E}^{2}$. 
4.6. Case of multiple disjoint biseps. Now consider a collection of pairwise disjoint, properly separating binodes $\Theta=\left(\underline{\theta}_{1}, \ldots, \underline{\theta}_{n}\right)$ of $X / B, \underline{\theta}_{i}=\left(\theta_{i, 1}, \theta_{i, 2}\right)$, corresponding to a mutually transverse collection of codimension-2 boundary loci $\partial_{i}=\partial_{\theta_{i, 1}} \cap \partial_{\theta_{i, 2}}$. Then the corresponding loci

$$
{ }_{\mathrm{L}} D\left(\underline{\theta}_{i}\right),{ }_{\mathrm{R}} D\left(\theta_{i}\right) \subset X_{B}^{[2]}, i=1, \ldots, n
$$

are also mutually transverse, so we may blow them up in any order, thus obtaining the azimuthal Hilbert scheme of $X / B$ associated to $\Theta$, which we denote by $X_{B}^{\{2\}}(\Theta)$. There is also an extended version, which is flat over $B(\Theta)$, the blowup in any order of $\partial_{1}, \ldots, \partial_{n}$, and is denoted $X_{B(\Theta)}^{\{2\}}$. As above, we obtain over $X_{B}^{\{2\}}(\underline{\theta})$ a mutually transverse collection of $2 n$ echelon data

$$
\left(\mathbb{E}^{-1,0}\left(\underline{\theta}_{i}\right),{ }_{\mathrm{L}} D^{\dagger}\left(\underline{\theta}_{i}\right)\right),\left(\mathbb{E}^{0,-1}\left(\underline{\theta}_{i}\right), \mathrm{R}_{\mathrm{R}} D^{\dagger}\left(\underline{\theta}_{i}\right)\right), i=1, \ldots, n
$$

from which we construct the associated echelon modification $\mathbb{E}_{\Theta}$, which comes with a map ('modified Brill-Noether'):

$$
\phi_{\Theta}: \mathbb{E}_{\Theta} \rightarrow \Lambda_{2}(\omega) .
$$

This of course factors $\phi_{\Theta^{\prime}}$ for any subset $\Theta^{\prime} \subset \Theta$. A similar construction can be made if $\Theta$ is a collection containing separating nodes (seps) and properly separating binodes (biseps) of $X / B$, all pairwise disjoint. As in $\$ 3.4$ it is easy to extend Lemma 4.9 to this situation and conclude

Proposition 4.11. Let $\Theta$ be a collection of pairwise disjoint seps and biseps of $X / B$, then for any azimuthal subscheme of $X / B$ contained in one separation component of $\Theta$, the image of the modified BrillNoether map $\phi_{\Theta}$ coincides that of the Brill-Noether map associated to the $\Theta$-sepcanonical system of X.

A nodal curve $X_{0}$ is said to be of semicompact type if all its biseps are maximal; equivalently, if the node set of $X_{0}$ is a disjoint union of seps, biseps, and absolutely nonseparating nodes.

Corollary 4.12. Let $X_{0}$ be a semicompact-type fibre of the versal family $X / B$, let $\Theta$ be the collection of all seps and biseps occurring on $\mathrm{X}_{0}$ and

$$
\phi_{\Theta}: \mathbb{E}_{\Theta} \rightarrow \Lambda_{2}(\omega)
$$

be the associated modification, defined over the azimuthal Hilbert scheme $X_{B}^{\{2\}}(\Theta)$. Then

$$
\mathbb{D}_{2}\left(\phi_{\Theta}\right)=\mathcal{H} \mathcal{E}^{\{2\}} \cup \underset{\theta \in \Theta \text { sep }}{\bigcup} R_{\theta}
$$

\section{Part 2. General case}

Here we deal with curves of non-semicompact type, i.e. curves admitting 'separating cycles' of nodes, herein called polyseparators. Constructing an appropriate modification of the Brill-Noether map to cover this case requires a more elaborate kind of blowup of the Hilbert scheme, related but not identical to the blowup induced by the natural number-of-nodes stratification of the boundary of the parameter space. This kind of blowup called stratified or normal blowup can be defined generally in the setting of a divisor with normal crossings. Applying it to the Hilbert scheme leads to the azimuthal Hilbert scheme. These constructions are pursued in $\$ 5$, Then in $\$ 6$ we construct the appropriate modification of the Brill-Noether map over the azimuthal Hilbert scheme and establish its basic properties. In $\$ 7$ we combine this result with an excess-intersection version of the Porteous formula to derive a formula for the fundamental class of the closure of 
the hyperelliptic locus (Theorem[7.2). $\sqrt{8}$ contains some of the intersection theory needed to make explicit the formula of Theorem 7.2 .

\section{MOdifying BRILl-NOETHER: POLYSEPARATORS}

5.1. S-Stratified blowup. Let $T$ be a divisor with local normal crossings on a smooth variety (or orbifold) $Y$ of dimension $n$, and let $S$ be a union of some irreducible components of the (orbifold) singular locus sing $(T)$, with the reduced structure, and some components of $T$ itself. Thus, each component of $S$ is either $(n-1)$ or $(n-2)$-dimensional. We assume $S$ has the following 'transitivity' property:

$\left.{ }^{*}\right)$ if $t_{1}, t_{2}, t_{3}$ define local branches of $T$ so that $\left(t_{1}, t_{2}\right)$ and $\left(t_{2}, t_{3}\right)$ define branches of $S$, then so does $\left(t_{1}, t_{3}\right)$.

Set $s_{2}(S)=S \cap \operatorname{sing}(T)$ and inductively,

$$
s_{i}(S)=\operatorname{sing}\left(s_{i-1}(S)\right), i>2
$$

(all with the reduced structure; singular locus understood in the orbifold sense). It is easy to see that $s_{i}(S)$ has codimension $i$ in $Y$ or is empty (e.g. if $i>n$ ) and that $s_{i}(S)$ is contained in the locus of points of multiplicity $\geq i$ on $T$. In fact, $s_{i}(S)$ coincides with the locus of points lying on $i$ local branches of $T$, every pair of which intersects in a branch of $S$. Thus a general point of $s_{i}(S)$ lies on $\left(\begin{array}{l}i \\ 2\end{array}\right)$ local analytic components of $S$. The chain

$$
s_{2}(S) \supset s_{3}(S) \ldots \supset s_{n}(S)
$$

is called the S-stratification of $Y$.

Example 5.1. Let $T=\delta_{0} \subset \overline{\mathcal{M}}_{\mathrm{g}}$, the divisor of curves with a nonseparating node, and let $\mathcal{S} \subset$ $\operatorname{sing}\left(\delta_{0}\right)$ denote the locus of curves with a separating binode. The associated $\mathcal{S}$-stratification, called the polyseparator stratification, has $i$-th stratum corresponding to curves with a degree- $i$ polyseparator.

Given $S$ as above, the stratified blowup of $Y$ associated to $S$, denoted

$$
B_{S}^{\sigma}(Y) \stackrel{b^{\sigma}}{\rightarrow} Y
$$

is the (smooth) variety obtained from $Y$ by first blowing up $s_{n}(S)$, then the (smooth) proper transform of $s_{n-1}(S)$, etc. (by definition, the blowup of the empty set is the identity $Y \rightarrow Y$ ). We denote by $E^{\sigma}(i)$ or $E_{S}^{\sigma}(i)$ the proper transform of $s_{i}(S)$ on $B_{S}^{\sigma}(Y)$, so that

$$
\left(b^{\sigma}\right)^{-1}\left(s_{i}(S)\right)=\sum_{j=n}^{i} E^{\sigma}(j)
$$

Clearly, $E^{\sigma}(i)$ is smooth and forms a locally trivial fibre bundle over the normalization of $s_{i}(S)$. The fibre, denoted $W_{i-1}^{\sigma}$, is a toric variety which may be identified with the stratified blowup of the union of the coordinate hyperplanes in $\mathbb{P}^{i-1}$. The structure of such bundles generally is described in 88.3 . 
5.2. Normal blowup. Here we describe an a-priori different, 'purely codimension-2' blowup construction that ultimately leads to the stratified blowup.

We continue the above notation. Locally, let $S_{1}, \ldots, S_{k}$ be all the local branches of $S$ through some point $p$. From the transitivity property, it follows that there are branches $T_{1}, \ldots, T_{r}$ of $T$ through $p$, such that $k=\left(\begin{array}{l}r \\ 2\end{array}\right)$ and the $S_{i}$ are the pairwise intersections $T_{j} \cap T_{\ell}, j \neq \ell$; in other words, locally at $p$, there is a normal-crossing subdivisor $T^{\prime}=\bigcup_{i=1}^{r} T_{i}$ such that

$$
S=\operatorname{sing}\left(T^{\prime}\right)
$$

Let

$$
\mathcal{J}=\prod_{i=1}^{k} I_{S_{i}}
$$

In fact, the ideal $\mathcal{J}$ is defined globally. We define the $S$-normal blowup of $Y$ as

$$
B{ }_{S}^{\perp}(Y):=\mathcal{B} \ell_{\mathcal{J}}(Y)
$$

Note that this coincides with the 'main' component of the fibred product $\prod\left(\mathcal{B} \ell_{S_{i}}(Y) / Y\right)$, i.e. the unique component dominating $Y$.

Our purpose is to prove:

Proposition 5.2. Notations as above, the S-stratified and normal blowups are equivalent over $Y$.

The Proposition will be proven below after some discussion. Its conclusion amounts to saying that the identity on $Y$ lifts to an isomorphism $B^{\perp} \stackrel{\sim}{\rightarrow} B^{\sigma}$. This assertion is local over $Y$, so replacing $T$ by the union of its branches through a given point and using the transitivity property, we may as well assume $S=\operatorname{sing}(T)$. Clearly, each irreducible component $S_{i}$ of $S$ pulls back to a Cartier divisor on $B^{\sigma}$, so by the universal property of blowing up we get a morphism $B^{\sigma} \rightarrow \mathcal{B}_{S_{i}}(Y)$. Putting these together yields a morphism $B^{\sigma} \rightarrow \Pi\left(\mathcal{B} \ell_{S_{i}}(Y) / Y\right)$, which as $B^{\sigma}$ is irreducible lands in the main component, whence a morphism

$$
B^{\sigma} \rightarrow B^{\perp} .
$$

To go the other way, we first study the normal blowup. We work locally analytically, so we can write

$$
S=\operatorname{sing}(T), T=\bigcup T_{i}, T_{i}=\left(t_{i}\right)
$$

where the $t_{i}$ are part of a regular sequence of parameters. For any index-set $I$, let

$$
T_{I}=\bigcap_{i \in I} T_{i}
$$

with ideal $\mathcal{J}_{I}$ locally generated by $t_{i}, i \in I$. Then we get the local factorization

$$
\mathcal{J}=\prod_{i<j} \mathcal{J}_{i j}, \mathcal{J}_{i j}=\left(t_{i}, t_{j}\right)
$$

Also, let $T(i)=\bigcup_{|I|=i} T_{I}$, and let $T(i)^{\text {norm }}$ be its normalization, which is smooth and locally a disjoint union of branches:

$$
T(i)^{\text {norm }}=\coprod_{|I|=i} T_{I}
$$


Let $T_{I}^{o}$ be the interior of $T_{I}$, i.e. $T_{I} \backslash \bigcup_{j \notin I} T_{j}$. For $i<j$, let $B_{i j}$ be the blowup of $T_{i j}=T_{i} \cap T_{j}$ in $Y$. Then we have

$$
B_{T}^{\perp}(Y) / Y=\prod_{i<j}^{\prime}\left(B_{i j} / Y\right)
$$

(where $\Pi^{\prime}$ means unique dominating component of fibre product over $Y$ ). Let $b: B_{T}^{\perp}(Y) \rightarrow Y$ be the natural map. For any $I$, let $E_{I}$ be the closure of $b^{-1}\left(T_{I}^{o}\right)$, and similarly for $E(i)$ and $E(i)$ norm.

To describe the structure of the exceptional divisors, we make the following construction. Let $L_{0}, \ldots, L_{n}$ be a collection of line bundles on a scheme $Z$, and let

$$
P_{i, j}=\mathbb{P}\left(L_{i} \oplus L_{j}\right), G_{i, j}=P_{i, j} \backslash\left(\mathbb{P}\left(L_{i}\right) \cup \mathbb{P}\left(L_{j}\right)\right), 0 \leq i<j \leq n .
$$

We identify $G_{i, j}$ with the $\mathbb{C}^{*}$ bundle associated to $L_{i}^{-1} \otimes L_{j}$. Consider the map

$$
\begin{array}{r}
\prod_{i=0}^{n-1} G_{i, i+1} \rightarrow \prod_{0 \leq i<j \leq n} P_{i, j} \\
\left(\lambda_{1}, \ldots, \lambda_{n}\right) \mapsto\left(\lambda_{i} \cdots \lambda_{j-1}: 0 \leq i<j \leq n\right), \lambda_{0}:=1
\end{array}
$$

and let $W\left[L_{0}, \ldots, L_{n}\right]$ be the closure of its image, obviously a fibre bundle over $Z$ with fibre a toric $n$-fold $W_{n}=W[\mathbb{C}, \ldots, \mathbb{C}](n+1$ factors, where $\mathbb{C}$ denotes the trivial line bundle over a point).

It is easy to see that $W\left[L_{0}, \ldots, L_{n}\right]$ can also be realized as the closed image of the rational map

$$
\begin{aligned}
\mathbb{P}\left[L_{0} \oplus \ldots \oplus L_{n}\right] & \rightarrow \prod_{0 \leq i \leq j \leq n} P_{i, j}, \\
{\left[x_{0}, \ldots, x_{n}\right] } & \rightarrow\left(\left[x_{i}, x_{j}\right]: 0 \leq i \leq j \leq n\right) .
\end{aligned}
$$

While the former description is better suited for the Lemma that follows, the latter one is more convenient for further study (see $\$ 8.3$ below). Note that $W\left[L_{0}, \ldots, L_{n}\right]$ is independent of the order of the line bundles. By abuse of notation, we will denote $W\left[L_{0}, \ldots, L_{n}\right]$ by $W\left[\bigoplus_{i=0}^{n} L_{i}\right]$ when the splitting of the rank- $(n+1)$ bundle is understood up to order.

Lemma 5.3. $\quad$ (i) $B_{T}^{\perp}(Y)$ is smooth.

(ii) $T(i)^{\text {norm }}$ is smooth, the inverse image $\tilde{T}(i+1)$ of $T(i+1)$ on it is a divisor with normal crossings. The map $E(i)^{\text {norm }} \rightarrow T(i)^{\text {norm }}$ factors through a map

$$
E(i)^{\text {norm }} \rightarrow B_{\tilde{T}(i+1)}^{\perp} T(i)^{\text {norm }}
$$

which is a locally trivial fibration with fibre $W_{i-1}$, of the form $W\left[N_{T(i)}\right.$ norm $\left.\rightarrow Y\right]$.

(iii) The reduced total transform of $T(i)$ on $B_{T}^{\perp}(Y)$ is $\bigcup_{i^{\prime} \geq i} E\left(i^{\prime}\right)$, and this is a divisor with normal crossings.

(iv) The exceptional divisor of $B_{T}^{\perp}(Y) \rightarrow Y$ is $\bigcup_{i \geq 2} E(i)$.

Proof. (i) Working locally, we cover the blowup with $2^{r}$ open sets $U$, each specified by a choice of ordering $i \rightarrow j$ or $t_{i} \rightarrow t_{j}$ on each integer pair $\{i, j\} \subset[1, r]$, indicating that $t_{j} / t_{i}$ is regular there. Then $\rightarrow$ generates a total order $\prec$, possibly with degeneracies or equivalences. Let $i_{0}$ be a minimum, unique up to equivalence. Then for any $i \prec j, j$ is reachable from $i$ by a chain of immediate- successor pairs and equivalent pairs. For an equivalent pair $a \sim b$, clearly $t_{a} / t_{b}$ is a 
unit. For any immediate successor $a \prec b$ we may up to equivalence assume $a \rightarrow b$. Thus, we may choose a set $P$ consisting of a maximal collection $P_{1}$ of immediate successors plus a suitable collection $P_{2}$ of equivalent pairs, $k-1$ from each equivalence class of cardinality $k$, such that for any $i \prec j$ (and this includes $\left.i_{0} \prec j, \forall j=1, \ldots, r\right), j$ is reachable from $i$ by a succession of pairs in $P$. Clearly $P$ has $n-1$ elements. Then $\left(t_{i_{0}}, t_{i} / t_{j}:(i, j) \in P_{1}\right)$ is a regular system of parameters on the blowup. These together with the units corresponding to $P_{2}$ and complementary coordinates to the $t_{i}$ yield a coordinate system on $U$.

(ii) The proper transform of $T_{I}$ in the blowup is locally the zero locus of $t_{i} / t_{j}, \forall i \in I, j \notin I$. This admits a forgetful map, forgetting the ratios $t_{i} / t_{i^{\prime}}, i, i^{\prime} \in I$, which clearly lands in the space of the $t_{j}$ and $t_{j} / t_{j^{\prime}}, j, j^{\prime} \notin I$, i.e. the normalized blowup $B_{\left(T_{j} \mid T_{I}: j \notin I\right)}^{\perp} T_{I}$, and has all fibres $W_{|I|-1}$.

(iii) and (iv): Straightforward, given the analysis above. Note that the local branches of $T(i)$ define a splitting of the normal bundle with summands defined up to order, as required in the definition above. Note that in the schematic total transform of $T(i)$, the $E\left(i^{\prime}\right)$ with $i^{\prime}>i$ will appear with higher multiplicities due to $T\left(i^{\prime}\right)$ lying on multiple branches of $T(i)$.

Remark 5.4. The mimimal index $i_{0}$ and corresponding minimal coordinate $t_{i_{0}}$ are of importance in their own right and will be used in the sequel.

Proof of Proposition 5.2 It remains to show there is a morphism $B^{\perp} \rightarrow B^{\sigma}$. This follows easily if we show that each $i$-dimensional stratum of the $S$-stratification pulls back to a Cartier divisor on $B^{\perp}$. We use the notations developed in the proof of the Lemma. Then, on a suitable open set and after rearranging so that the ordering of the coordinates is the standard one $1 \prec 2 \ldots \prec r$, the $i$-dimensional stratum, defined in $Y$ by $t_{i+1}, \ldots, t_{r}$, pulls back to the Cartier divisor in $B^{\perp}$ defined by $t_{i+1}$, because $\left[t_{i+2} / t_{i+1}\right], \ldots,\left[t_{r} / t_{i+1}\right]$ are regular. This concludes the proof.

5.3. Azimuthal blowup. We extend the notion of left azimuthal modification of 44.1 to the case of multiple, not necessarily disjoint, binodes. This again will be needed primarily for the sake of comparing a modified Brill-Noether map with an ordinary one associated to a sepcanonical system. The construction will be local over the base, so fix a fibre $X_{0}$ of $X / B$, and let $\Theta=\Theta_{1} \cup \Theta_{2}$ be a set of seps $\theta_{i} \in \Theta_{1}$ and biseps $\underline{\theta}_{i} \in \Theta_{2}$ occurring on $X_{0}$, and assume $\Theta_{2}$ has the transitivity property as in $\$ 5.1$. Let $Y$ be a component of the separation $X_{0}^{\Theta}$, and assume that all the elements of $\Theta$ are oriented so as to have $Y$ on their left (we call this a $Y$-compatible orientation). Let $B^{\sigma}(\Theta)$ denote the normal (=stratified) blowup of $B$ corresponding to $\Theta_{2}$. This is virtually smooth, i.e. smooth if $B$ is smooth and $X / B$ is versal, and is independent of orientations. Also $B^{\sigma}(\Theta)$ maps to the blowup $B(\underline{\theta}), \forall \underline{\theta} \in \Theta_{2}$. When $\Theta$ coincides with the set of all seps and biseps of $X_{0}$ we will omit $\Theta$. Locally, branches of the exceptional locus of $B^{\sigma}(\Theta) / B$ correspond to polyseparators $\Pi \subset\left|\Theta_{2}\right|:=\bigcup_{\underline{\theta} \in \Theta_{2}} \underline{\theta}$ and we denote the branch corresponding to $\Pi$ by $\Xi(\Pi)$.

Note that a point $0^{\prime} \in B^{\sigma}(\Theta)$ over $0 \in B$ corresponds to a collection of middle azimuths $\mathrm{M} \zeta(\underline{\theta}), \forall \underline{\theta} \in \Theta_{2}$, subject to relations coming from maximal polyseparators. The point $0^{\prime}$ is said to be regular if all the ${ }_{M} \zeta(\underline{\theta})$ are regular. This holds if and only if $0^{\prime}$ sits only on branches $\Xi(\Pi)$ where $\Pi$ is a maximal polyseparator on $X_{0}$. 
Let $X_{B^{\sigma}(\Theta)}$ be the base changed family. This contains Cartier divisors $X_{\Xi(\Pi)}$ for polyseparators $\Pi$. The latter splits as a union of Weil divisors, where $\Pi=\left(\theta_{1}, \ldots, \theta_{n}\right)$ (cyclical arrangement):

$$
X_{\Xi(\Pi)}=\bigcup_{i=1}^{n} \mathrm{~L} X\left(\theta_{i}, \theta_{i+1}\right)_{\Xi(\Pi)} .
$$

To describe this in local coordinates, let $t_{i}=x_{i} y_{i}$ be a local equation for $\partial_{\theta_{i}}, i=1, \ldots, n$, and recall that at each point of $\Xi(\Pi)$, there is a 'minimal' index $j$ so that $B^{\sigma}(\Theta)$ admits local parameters

$$
t_{1} / t_{j}, \ldots, t_{j-1} / t_{j}, t_{j}, t_{j+1} / t_{j}, \ldots, t_{n} / t_{j}
$$

and $t_{j}$ is an equation for $\Xi(\Pi)$. At $\theta_{i},{ }_{\mathrm{L}} X\left(\theta_{i}, \theta_{i+1}\right)_{\Xi(\Pi)}$ is either Cartier, if $i=j$, or has local equations $y_{i}, t_{j}$ if $j \neq i$.

The ${ }_{L} X\left(\theta_{i}, \theta_{i+1}\right)_{\Xi(\Pi)}$ are mutually transverse for fixed $\Pi$ (in fact, three distinct ones have empty intersection). Clearly they are also mutually transverse for distinct $\Pi$ (as the divisors $\Xi(\Pi)$ are already transverse). Then consider their joint blowup, i.e. the blowup of the ideal sheaf $\prod_{i} \mathcal{I}_{\mathrm{L} X\left(\theta_{i}, \theta_{i+1}\right)_{\Xi(\Pi)}}$, which coincides with the unique dominant component of the fibre product $\prod_{i} \stackrel{i}{\mathcal{B}} \ell_{\mathrm{L}} X\left(\theta_{i}, \theta_{i+1}\right)_{\Xi(\Pi)} X_{B^{\sigma}(\Theta)}$. We denote this common blowup by ${ }_{\Upsilon} X_{B^{\sigma}(\Theta)}$ and call it the azimuthal modification of $X / B$ corresponding to $Y$. It depends on $Y$ only for orientation, and is defined over a neighborhood of the fibre $X_{0}$ containing $Y$ and defines a family of curves over a neighborhood of $0 \in B$. As in 4.1 , it is virtually smooth. It comes equipped with a birational morphism over $B(\Theta)$

$$
{ }_{B^{\sigma}(\Theta)} \rightarrow X_{B^{\sigma}(\Theta)}
$$

Now suppose that $0^{\prime} \in B^{\sigma}(\Theta)$ is a regular point as above, hence lies only on divisors $\Xi(\Pi)$ where $\Pi$ is a maximal polyseparator on $X_{0}$. Given any bisep $\underline{\theta}$ (as always, oriented with $Y$ to its left) on $X_{0}$, it is contained as an adjacent pair in a unique maximal polyseparator $\Pi(\underline{\theta})$, and we let

$$
{ }_{Y} X(\underline{\theta})={ }_{L} X(\underline{\theta}) \Xi(\Pi(\underline{\theta}))
$$

be the corresponding (Cartier) divisor. Additionally, we have for each sep $\theta$ on $X_{0}$, a divisor ${ }_{Y} X(\theta)$ on ${ }_{Y} X_{B^{\sigma}(\Theta)}$ which is the pullback of ${ }_{\mathrm{L}} X(\theta)$. Then on ${ }_{Y} X_{B^{\sigma}(\Theta)}$ we may consider the twisted canonical bundle

$$
\gamma \omega^{\text {sep }}=\gamma \omega^{\operatorname{sep}}\left({ }_{\mathrm{M}} \zeta_{\bullet}\right):=\omega_{\gamma_{B^{\sigma}(\Theta)} / B^{\sigma}(\Theta)}\left(-2 \sum_{\theta_{i} \in \Theta_{1}} \gamma X\left(\theta_{i}\right)-\sum_{\underline{\theta}_{i} \in \Theta_{2}} \gamma X\left(\underline{\theta}_{i}\right)\right)
$$

where ${ }_{M} \zeta_{\bullet}$ is the collection of (regular) middle azimuths corresponding to $0^{\prime}$ (and will be suppressed when understood). We call this the relative sepcanonical system adapted to $Y$. Proposition 6.10 of [18] can now be generalized as follows

Proposition 5.5. For the above line bundle ${ }_{Y} \omega^{\mathrm{sep}}$, the image of the natural restriction map

$$
\pi_{*}\left(\Upsilon \omega^{\text {sep }}\right) \rightarrow H^{0}\left(Y, Y \omega^{\text {sep }} \otimes \mathcal{O}_{Y}\right)
$$

coincides with the restriction of the sepcanonical system $\left|\omega_{X}\right|^{\text {sep }}$ on $Y$.

Proof. Begin with some elementary remarks. First, $0^{\prime}$ being regular implies that any bisep that has at least 1 point on $Y$ actually has both points on $Y$. Moreover the various biseps occurring 
on $Y$ are disjoint, hence are contained in disjoint maximal polyseparators. Next, for any nonmaximal bisep $\underline{\theta}$ ocurring on $Y$ (and having $Y$ to its left), $\underline{\theta}$ is automatically non-right-hyperelliptic, hence imposes no azimuthal condition on $\mathrm{L} \underline{\theta}$.

Now to check necessity of the conditions defining $|\omega|^{\text {sep }}$, we can work as in the proof of Proposition 6.10 of [18], smoothing out all but one sep or bisep on $Y$, in which case the condition at the remaining one becomes obvious. This proves necessity. The proof of sufficiency is identical to the corresponding argument in the proof of Proposition 6.10 of [18].

5.4. Azimuthal Hilb. Here we give the construction of an azimuthal modification of the Hilbert scheme by an appropriate stratified blowup. This is the construction we will really use. It is related to that of 55.3 , but differs from it in being defined already over $B$ itself. We shall work here with oriented biseps and their left sides only, but since each plain bisep will occurs twice with opposite orientations, the eventual construction will be symmetric.

5.4.1. The definition. We fix the versal family $X / B$ as before. Here we put together the per-binode modifications of the Hilbert scheme described in $\$ 4.2$. Given an oriented bisep $\underline{\theta}$, usually defined only locally over $B$, let

$$
S_{\underline{\theta}}={ }_{\mathrm{L}} X(\underline{\theta})^{[2]}
$$

which is a subset of $\operatorname{sing}(T)$, where $T$ is the pullback on $X_{B}^{[2]}$ of the divisor $\delta_{0}(B)$ of generically irreducible nodal curves. For a good family $X / B$ (cf. $\left.\oint_{2}\right), T$ has normal crossings. Then let

$$
S=\bigcup_{\underline{\theta}} S_{\underline{\theta}}
$$

the union being over all oriented biseps $\underline{\theta}$ (each unoriented bisep will appear twice, so both its sides will appear). Then $S$ is globally defined over $B$, and we define the azimuthal Hilbert scheme as the stratified (or equivalently, normal, see Proposition 5.2) blowup:

$$
X_{B}^{\{2\}}=B_{S}^{\sigma}\left(X_{B}^{[2]}\right) \stackrel{b}{\rightarrow} X_{B}^{[2]} .
$$

Elements of $X_{B}^{\{2\}}$ will be referred to as 'azimuthal schemes. By construction, $X_{B}^{\{2\}}$ dominates every $X_{B}^{\{2\}}(\underline{\theta})$ over the open set of $B$ where the latter is defined, and also dominates the analogous blowup corresponding to any collection of binodes where the collection is defined. Therefore an azimuthal scheme, whose underlying 'plain' scheme is disjoint from all biseps, may be viewed as a scheme with a collection of middle azimuths ${ }_{\mathrm{M}} \zeta(\underline{\theta})$, one for each oriented bisep $\underline{\theta}$ having $z$ on its left. These azimuths are not independent but are subject to relations of compatibility 'around maximal polyseparators' (see the proof of Proposition 5.2).

5.4.2. Exceptional divisors. We aim to describe the exceptional locus of this blowup, first locally over $B$. By the general description above, the components of the exceptional divisor correspond to components of the various strata (of different dimensions) of the stratification corresponding to $S$. Thus, consider a singular fibre $X_{0}$ and a maximal oriented polyseparator $\Theta_{\max }$ on $X_{0}$ and adjacent bisep in it

$$
\underline{\theta}=\left(\theta_{1}, \theta_{2}\right) \subset \Theta_{\max } .
$$

Note that $X_{0}$ and $\underline{\theta}$ determine $\Theta_{\max }$. Then set

$$
Y_{0}=\underset{35}{L} X_{0}(\underline{\theta}) .
$$


There is a codimension- 2 boundary locus $\partial_{\underline{\theta}} \subset B$ in a neighborhood of 0 over which $\underline{\theta}$ is defined, and $Y_{0}$ extends to a family $Y=Y_{\underline{\theta}} / \partial_{\underline{\theta}}={ }_{\mathrm{L}} X_{\underline{\theta}}(\underline{\theta})$, in whose generic member the opposite side ${ }_{\mathrm{R}} X_{\underline{\theta}}(\underline{\theta})$ is irreducible, as is $Y_{\underline{\theta}}$. Note $Y_{0}$ extends as well as to smaller subfamilies

$$
Y_{\underline{\theta}, \Theta}=Y_{\underline{\theta}} \times \partial_{\underline{\theta}} \partial_{\Theta}
$$

one for each $\Theta$ which is a polyseparator on $X_{0}$ containing $\underline{\theta}$ and contained in $\Theta_{\max }$. The family $Y_{\underline{\theta}, \Theta}$ does not depend on $\Theta_{\max }$. It is locally defined on $X$ by the equations defining ${ }_{\mathrm{L}} X\left(\theta_{1}\right)$ and ${ }_{\mathrm{L}} X\left(\theta_{2}\right)$ plus base equations defining $\partial_{\theta}, \forall \theta \in \Theta \backslash \underline{\theta}$. For a general fibre over $\partial_{\Theta}, \Theta$ is a maximal polyseparator and $\underline{\theta}$ is adjacent on it. Each such family $Y_{\underline{\theta} \Theta}$ gives rise to a divisor $\Xi_{\underline{\theta}}(\Theta)$, equal to the proper transform of $Y_{\underline{\theta} \Theta}^{[2]}$ on $X_{B}^{\{2\}}$. By Lemma 5.3 and Lemma 4.3, the restricted blowdown $\operatorname{map} \Xi_{\underline{\theta}}(\Theta) \rightarrow Y_{\underline{\theta}, \Theta}^{[2]}$ is a $W_{n-1}$-bundle, of the form

$$
\Xi_{\underline{\theta}}(\Theta)=W_{\gamma_{\underline{\theta}}^{[2]}}\left[\theta_{1}^{\dagger}, \theta_{2}^{\dagger},-\partial_{\theta_{3}}, \ldots,-\partial_{\theta_{n}}\right]
$$

where $\Theta=\left(\theta_{1}, \ldots, \theta_{n}\right)$ is a cyclic arrangement and

$$
\theta_{i}^{\dagger}=\mathcal{O}\left(-\partial_{\theta_{i}}+[2]_{*}\left({ }_{\mathrm{L}} \theta_{i}\right)\right), i=1,2 .
$$

In particular, there is a projection

$$
p: \Xi_{\underline{\theta}}(\Theta) \rightarrow \mathbb{P}_{\gamma_{\underline{\theta} \Theta}^{[2]}}\left(\theta_{1}^{\dagger} \oplus \theta_{2}^{\dagger} \oplus \mathcal{O}\left(-\partial_{\theta_{3}}\right) \oplus \ldots \oplus \mathcal{O}\left(-\partial_{\theta_{n}}\right)\right)
$$

Let

$$
L_{\underline{\theta}}(\Theta)=p^{*}(\mathcal{O}(1)) .
$$

Also, if $\Theta^{\prime}$ is any polyseparator between $\underline{\theta}$ and $\Theta$, i.e. with $\underline{\theta} \subset \Theta^{\prime} \subset \Theta$, we have a map

$$
p_{\Theta^{\prime}}: \Xi_{\underline{\theta}}(\Theta) \stackrel{q_{\Theta^{\prime}}}{\rightarrow} W_{\gamma_{\underline{\theta}}^{[2]}}\left[\theta_{1}^{\dagger}, \theta_{2}^{\dagger},-\partial_{\theta_{i}}: \theta_{i} \in \Theta^{\prime}\right] \rightarrow \mathbb{P}\left(\theta_{1}^{\dagger} \oplus \theta_{2}^{\dagger} \oplus-\partial_{\theta_{i}}: \theta_{i} \in \Theta^{\prime}\right)
$$

whence a line bundle

$$
L_{\underline{\theta}}\left(\Theta, \Theta^{\prime}\right)=p_{\Theta^{\prime}}^{*}(\mathcal{O}(1)) .
$$

Thus,

Also, we have locally near 0 ,

$$
L_{\underline{\theta}}\left(\Theta, \Theta^{\prime}\right)=q_{\Theta^{\prime}}^{*}\left(L_{\underline{\theta}}\left(\Theta^{\prime}\right) .\right.
$$

Because $\Xi_{\underline{\theta}}$ is the pullback of ${ }_{\mathrm{L}} D^{\dagger}(\underline{\theta})$ from $X_{B}^{\{2\}}(\underline{\theta})$, we have by (4.3.4) and (4.3.5) that

$$
\mathcal{O}_{\Xi_{\underline{\theta}}}\left(-\Xi_{\underline{\theta}}\right)=\mathcal{O}_{\mathcal{O}\left(\theta_{1}^{\dagger}\right) \oplus \mathcal{O}\left(\theta_{2}^{\dagger}\right)}(1) \text {. }
$$

Then consider, for any $n \geq 2$ :

$$
\Xi(n)=\sum_{\underline{\theta}} \sum_{\substack{\theta \subset \Theta \\|\Theta|=n}} \Xi_{\underline{\theta}}(\Theta) .
$$

This is independent of any branch choices and extends to a global divisor on $X_{B}^{\{2\}}$. 
In terms of local equations, if $X / B$ is given locally near $\theta_{i}$ by $x_{i} y_{i}=t_{i}$, where $y_{1}, y_{2}$ are local coordinates on $Y_{0}$ near ${ }_{\mathrm{R}} \theta_{1},{ }_{\mathrm{R}} \theta_{2}$ respectively, then $Y_{\underline{\theta} \Theta \Theta}$ is defined near $\left(\theta_{1}, \theta_{2}\right)$ by $\left(x_{1}, x_{2}, t_{i}, i \in\right.$ $\Theta \backslash \underline{\theta})$. Therefore the divisor $\sum_{\Theta_{1} \supset \Theta} \Xi_{\underline{\theta}}\left(\Theta_{1}\right)$ is locally defined in various covering opens upstairs in $X_{B}^{\{2\}}$ by the minimum of these coordinates, in the sense of Remark 5.4 (the minimum varies from one open to the other). This minimum also corresponds to a local generator of $L_{\underline{\theta}}(\Theta)$, and consequently the conormal bundle

$$
\mathcal{O}_{\Xi_{\underline{\underline{\theta}}}(\Theta)}\left(-\Xi_{\underline{\theta}}(\Theta)\right)=L_{\underline{\theta}}(\Theta) \otimes \mathcal{O}_{\Xi_{\underline{\theta}}(\Theta)}\left(\sum_{\substack{\Theta_{1} \\ \Theta_{1 \supsetneq} \supseteqq}} \Xi_{\underline{\theta}}\left(\Theta_{1}\right)\right) .
$$

We summarize the above discussion as follows.

Theorem 5.6. (i) The map $X_{B}^{\{2\}} \rightarrow X_{B}^{[2]}$ is birational, with exceptional divisor $\Xi=\sum_{n \geq 2} \Xi(n)$ which decomposes locally as

$$
\Xi=\sum_{\Theta \text { polyseparator }} \sum_{\underline{\theta} \subset \Theta \text { adjacent }} \Xi_{\underline{\theta}}(\Theta) .
$$

(ii) $X_{B}^{\{2\}}$ is 'virtually smooth', i.e. smooth when $X / B$ is a good family.

(iii) The boundary of $X_{B}^{\{2\}} / B$ is a divisor with local normal crossings.

(iv) Each $\Xi_{\underline{\theta}}(\Theta), \Theta=\left(\theta_{1}, \ldots, \theta_{n}\right)$, is a $W_{n-1}$-bundle fibration induced by a birational base-change from a bundle of the form

$$
W\left[\theta_{1}^{\dagger}, \theta_{2}^{\dagger},-\delta_{3}, \ldots,-\delta_{n}\right] \rightarrow Y_{B_{\Theta}}^{[2]}
$$

where $Y$ is locally a 2-inseparable component subfamily of $X_{B_{\Theta}}$ which is one side of the separating binode $\underline{\theta} \underline{\theta} \subset \Theta,-\delta_{i}$ is the ideal of the branch of the boundary corresponding to $\theta_{i}$ and $\theta_{i}^{\dagger}=-\delta_{i}+\theta_{i}$.

(v) The self-intersection of $\Xi_{\underline{\theta}}(\Theta)$ is

$$
-c_{1}\left(L_{\underline{\theta}}(\Theta)\right)-\left(\sum_{\substack{\Theta \text { adjacent on } \Theta_{1} \\ \Theta_{\underline{\theta}}}} \Xi_{\underline{\theta}}\left(\Theta_{1}\right)\right) \cdot \Xi_{\underline{\theta}}(\Theta)
$$

(see (5.4.3)).

Remark 5.7. To be precise, $\Xi_{\underline{\theta}}(\Theta)$ is a fibration of the form $W[\underline{\theta} ; \Theta]:=W\left[\theta_{1}^{\dagger}, \theta_{2}^{\dagger},-\delta_{3}, \ldots,-\delta_{n}\right]$ over the 'induced azimuthal blowup' of $Y_{B_{\Theta}}^{[2]}$ which is the normal blowup corresponding to $\Theta_{\max } \backslash \Theta$. The exceptional divisor in this blowup is the sum of the divisors $\Xi_{\underline{\theta}}\left(\Theta_{1}\right) . \Xi_{\underline{\theta}}(\Theta)$ appearing in 5.4.8. The divisor $\Xi_{\underline{\theta}}\left(\Theta_{1}\right) \cdot \Xi_{\underline{\theta}}(\Theta)$ (on $\Xi_{\underline{\theta}}(\Theta)$ ) is in itself a birational pullback of $W$ bundle of the form $W[\underline{\theta} ; \Theta]$ over a birational pullback of $Y_{B_{\Theta_{1}}}^{[2]}$. Note that two such divisors $\Xi_{\underline{\theta}}\left(\Theta_{1}\right) . \Xi_{\underline{\theta}}(\Theta), \Xi_{\underline{\theta}}\left(\Theta_{2}\right) . \Xi_{\underline{\theta}}(\Theta)$ are disjoint unless $\Theta_{1} \subset \Theta_{2}$ or vice versa, because they are both proper transforms in a blowup of a smaller stratum corresponding to $\Theta_{1} \cup \Theta_{2}$. If $\Theta_{1} \varsubsetneqq \Theta_{2}$ then the intersection $\Xi_{\underline{\theta}}\left(\Theta_{1}\right) \cdot \Xi_{\underline{\theta}_{\hat{\theta}}}\left(\Theta_{2}\right) . \Xi_{\underline{\theta}}(\Theta)$ is a fibre product $W\left[\underline{\theta}, \Theta_{1}\right] \times W\left[\Theta_{2} \backslash \Theta_{1}\right]$ over a birational pullback of $Y_{B_{\Theta_{2}}}^{[2]}$. 
More generally an intersection $\bigcap_{i=1}^{m}\left(\Xi_{\underline{\theta}}\left(\Theta_{i}\right) \cdot \Xi_{\underline{\theta}}(\Theta)\right)$ is empty unless the polyseparator collection $\left\{\Theta_{1}, \ldots, \Theta_{m}\right\}$ is totally ordered under inclusion, and if we label them so that $\Theta_{1} \varsubsetneqq \ldots \varsubsetneqq \Theta_{m}$ then the intersection is a fibre product

$$
W\left[\underline{\theta} ; \Theta_{1}\right] \times W\left[\Theta_{2} \backslash \Theta_{1}\right] \times \ldots W\left[\Theta_{m} \backslash \Theta_{m-1}\right]
$$

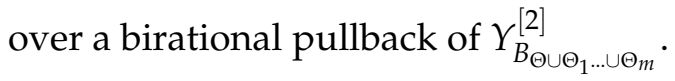

Example 5.8. For the universal family of curves over $\overline{\mathcal{M}}_{\mathrm{g}}$, the choice of $\underline{\theta}$ and $\Theta$ corresponds to writing $g=g_{1}+\ldots+g_{n}+1$ and looking at the boundary locus corresponding to a cyclical arrangement of 2-pointed curves glued cyclically along the marked points, so that $\left.{ }_{\mathrm{L}} X\left(\theta_{i}, \theta_{i+1}\right),{ }_{\mathrm{L}} \theta_{i},{ }_{\mathrm{L}} \theta_{i+1}\right)$ is the $i$-th marked curve. In particular, $Y(\underline{\theta}), \underline{\theta}=\left(\theta_{1}, \theta_{2}\right)$ is then the universal 2-pointed curve over $\mathcal{M}_{g_{1}, 2}$, pulled back over $\mathcal{M}_{g_{1}, 2} \times \mathcal{M}_{g_{2}, 2} \times \ldots \times \mathcal{M}_{g_{n}, 2}$ and $B l_{\underline{\theta}}\left(Y(\underline{\theta})_{B(\Theta)}^{\{2\}}\right)$ is the appropriate modification of its Hilbert scheme. We may identify $\Xi_{\underline{\theta}}(\Theta)$ with the $W_{n-1}$-bundle $W\left[\psi_{1} \otimes\right.$ $\left.\mathcal{O}\left([2]_{*} \theta_{1}\right) \oplus \psi_{2} \otimes \mathcal{O}\left([2]_{*} \theta_{2}\right) \oplus \bigoplus_{i=3}^{n} \psi_{i}\right]$ where $\psi_{i}={ }_{\mathrm{L}} \psi\left(\theta_{i}\right) \otimes{ }_{\mathrm{R}} \psi\left(\theta_{i}\right)$. See Section 8.3 for more information on this.

Example 5.9. Consider the extremal case of an n-dimensional family with an isolated 'cyclic' fibre of the form $X_{0}=Y_{1} \cup \ldots \cup Y_{n}$. We get a polyseparator $\Theta_{\max }=\left(\theta_{1}, \ldots, \theta_{n}\right)$ and adjacent biseps $\underline{\theta}_{i}=\left(\theta_{i}, \theta_{i+1}\right)$ and the line bundles $\partial_{\theta_{i}}$ are all trivial. Hence $\Xi_{\underline{\theta}_{i}}(\Theta)$ is a $W_{n-1}$-bundle of the form $W\left[L_{i}, L_{i+1}, \mathcal{O}, \ldots, \mathcal{O}\right]$ where $L_{i}=[2]_{*}\left(\mathcal{O}\left(-{ }_{L} \theta_{i}\right)\right)$. For example, $W_{2}$ is the blowup of $\mathbb{P}^{2}$ in 3 points and the above $W_{2}$-bundle is the blowup of $\mathbb{P}\left(L_{i} \oplus L_{i+1} \oplus \mathcal{O}\right)$ in the 3 distinguished sections. Because $\Theta$ is maximal, the conormal bundle of $\Xi_{\underline{\theta}_{i}}(\Theta)$ is just $L_{\underline{\theta}_{i}}(\Theta)$.

\section{Azimuthal BRill Noether AND SEPCANONiCAl SYSTEM}

In this section we will state and prove our definitive result on the modified Brill-Noether map and its relation to the sepcanonical system.

6.1. Azimuthal Hodge bundle. To this end we will construct an echelon modification of the Hodge bundle over the full azimuthal Hilbert scheme $X_{B}^{\{2\}}$. First working locally near a singular fibre $X_{0}$, assumed oriented, consider the following collection of echelon data on $X_{B}^{[2]}$ :

- $\chi(\theta)$, for all separating (relative) nodes $\theta$ that meet (i.e. occur on) $X_{0}$ (see (3.1.3));

- $\chi(\underline{\theta}):=\left(\mathbb{E}^{0,-1}(\underline{\theta}), \Xi_{\underline{\theta}}\right)$, where $\mathbb{E}^{0,-1}$ is as in (4.4.2) and $\Xi_{\underline{\theta}}$ is as in (5.4.5) for all oriented biseps $\underline{\theta}$ meeting $X_{0}$ (each oriented bisep will occur twice in this list, with its two orientations).

By Theorem 5.6, $\sum_{\underline{\theta}} \Xi_{\underline{\theta}}$ is a divisor with normal crossings. Therefore these echelon data are mutually transverse. Moreover, the entire collection- though not its individual members - is canonically and globally defined. Therefore this collection is a collection of polyechelon data in the sense of [16], §3. Therefore, there is globally defined over the azimuthal Hilbert scheme $X_{B}^{\{2\}}$ an associated modification which we call the azimuthal Hodge bundle ${ }_{a} \mathbb{E}$. It comes together with a map called the azimuthal Brill-Noether map associated to $X / B$ :

$$
{ }_{\mathfrak{a}} \phi:{ }_{\mathfrak{a}} \mathbb{E} \rightarrow \Lambda_{2}(\omega) \text {. }
$$


6.2. Comparison. The first order of business with ${ }_{a} \phi$ is to derive for it a comparison diagram analogous to (3.2.1) and (4.5.2). This diagram is local, and depends on a choice of fibre of $X / B$ as well as a 2-component of it.

Thus, let $X_{0}$ be a fibre and $Y$ a 2-component of $X_{0}$, i.e. a connected component of the blowup of $X_{0}$ in the collection $\Theta$ of all seps and biseps (maximal or not, on or off $Y$ ). We assume $X_{0}$ is oriented so that $Y$ is to the left of each $\operatorname{sep} \theta$ and bisep $\underline{\theta}$. Set

$$
X^{\{2\}}(Y)=\left(X_{B}^{\{2\}} \times_{B} \prod_{\text {bisep } \underline{\theta}}\left(X_{L}\right)_{B(\underline{\theta})}^{[2]}\right)^{\prime}
$$

where as usual ' refers to the unique component of the fibre product over $B$ which dominates $B$. This is an analogue of the space denoted $Y$ in (4.5.1). Then $X^{\{2\}}(Y)$ admits a natural map to $B^{\perp}$, the normal blowup of $B$ associated to the family of $B(\underline{\theta})$; as well as to the various factors, via which we may pull back various objects defined on these factors. Set

$$
\begin{gathered}
{ }_{\mathrm{L}} D_{1}^{\dagger}(Y)=\sum_{\operatorname{seps} \theta}{ }_{\mathrm{L}} D^{\dagger}(\theta),{ }_{\mathrm{L}} D_{2}^{\dagger}(Y)=\sum_{\text {biseps } \underline{\theta}}{ }_{\mathrm{L}} D^{\dagger}(\underline{\theta}), \\
{ }_{\mathrm{L}} X_{1}(Y)=\sum_{\operatorname{seps} \theta}{ }_{\mathrm{L}} X(\theta),{ }_{\mathrm{L}} X_{2}(Y)=\sum_{\text {biseps } \underline{\theta}}{ }_{\mathrm{L}} X_{P}(\underline{\theta}) .
\end{gathered}
$$

We let $Y^{\{2\}} \subset X_{B}^{\{2\}}$ denote the inverse image of $Y^{[2]} \subset X_{B}^{[2]}$. Thus, an interior element $z$ of $Y^{\{2\}}$ consists of an interior subscheme of $Y$ together with a collection of middle azimuths at all biseps on $X_{0}$ (on or off $Y$ ). In particular, $z$ induces on $X_{0}$ a structure of azimuthal curve. We have, for each sep $\theta$,

$$
Y^{\{2\}} \subset{ }_{\mathrm{L}} D^{\dagger}(\theta)
$$

Note that the map $X^{\{2\}}(Y) \rightarrow X_{B}^{\{2\}}$ is an isomorphism over the locus of 'interior' schemes, i.e. those disjoint from all biseps. Now set

$$
\mathbb{E}^{Y}=\left(\pi_{*}\left(\omega\left(-2{ }_{\mathrm{L}} X_{1}(Y)-{ }_{\mathrm{L}} X_{2}(Y)\right)\right)\right)_{X^{\{2\}}(Y)} .
$$

Then we get a comparison diagram analogous to (4.5.2):

$$
\begin{array}{cc}
\mathbb{E}^{Y}\left(2_{\mathrm{L}} D_{1}^{\dagger}(Y)+{ }_{\mathrm{L}} D_{2}^{\dagger}(Y)\right) & \rightarrow \\
\downarrow & \circlearrowleft{ }_{\mathfrak{a}} \mathbb{E} \\
\Lambda_{2}\left(\omega\left(-2{ }_{\mathrm{L}} X_{1}(Y)-{ }_{\mathrm{L}} X_{2}(Y)\right)\right)\left({ }_{2} D_{1}^{\dagger}(Y)+{ }_{\mathrm{L}} D_{2}^{\dagger}(Y)\right) & \rightarrow \Lambda_{2}(\omega) .
\end{array}
$$

Again the left column is up to an isomorphism (namely a twist) the Brill-Noether map associated to the bundle $\omega\left(-2{ }_{\mathrm{L}} X_{1}(Y)-{ }_{\mathrm{L}} X_{2}(Y)\right)$. We will be using this diagram mainly over $Y^{\{2\}}$. A few remarks are in order.

(i) If $\theta$ is a *-sep (i.e. sep or bisep) disjoint from $Y$, with associated divisor ${ }_{L} D^{\dagger}(\theta)$, which sits over a divisor $\partial_{\theta} \subset B^{\perp}$, then

$$
\left.{ }_{\mathrm{L}} D^{\dagger}(\theta)\right|_{Y\{2\}}=\left.\pi^{*}\left(\partial_{\theta}\right)\right|_{Y\{2\}}
$$

and similarly for ${ }_{L} X_{P}(\theta)$ etc. It follows that the left column of (6.2.3) is equivalent to a map where ${ }_{\mathrm{L}} D_{i}^{\dagger}(Y),{ }_{\mathrm{L}} X_{i}(Y)$ are replaced by the sum over those *-seps that meet $Y$.

(ii) The bottom arrow of (6.2.3) is an isomorphism over the interior of $Y^{\{2\}}$. 
(iii) Given a ${ }^{*}$-sep $\theta$ on $Y, \theta$ is right hyperelliptic on $X_{0}$ if and only if $\left({ }_{\mathrm{R}} X(\theta),{ }_{\mathrm{R}} \theta\right)$ is hyperelliptic as a pair; this has the consequence, nontrivial only if $\theta$ is a bisep, that ${ }_{R} \theta$ is contained in a single 2-component of ${ }_{\mathrm{R}} X(\theta)$, so that any 2-component of ${ }_{\mathrm{R}} X(\theta)$ is also a 2-component of $X_{0}$.

(iv) Given an interior scheme $z$ on $Y$, it induces azimuthal data ${ }_{M} \zeta_{\underline{\theta}}$ on all the biseps $\underline{\theta} \in \Theta$ and hence endows $Y$ with the restriction of the sepcanonical system $|\omega|_{\Theta}^{\text {sep }}$ from $X_{0}$, and the image of the associated Brill-Noether map coincides with that of the azimuthal Brill-Noether map a $\phi$ over $z$.

6.3. Main results. We recall that a 2-inseparable stable curve is hyperelliptic if it is a $2-1$ cover of $\mathbb{P}^{1}$ and that such curves are classified, e.g. in [18], $\S 2$. A stable hyperelliptic curve is a tree-like arrangement of 2-inseparable hyperelliptics, or equivalently, an admissible 2-1 cover of a rational tree. A semistable curve is hyperelliptic provided its stable contraction is. We now proceed to state our main results. First some notation. Denote by $\mathcal{H E}_{B}^{\{2\}}$ the closure of the inverse image in $X_{B}^{\{2\}}$ of the hyperelliptic pair locus $\mathcal{H}_{\mathcal{E}^{o}}^{2}$ over the set of smooth curves in $B$. Denote by $\mathrm{R}^{\mathrm{sep}}(X / B)$ the inverse image in $X_{B}^{\{2\}}$ of the locus of schemes supported on separating nodes of $X / B$. It has codimension 2 in $X_{B}^{\{2\}}$. The following result extends Proposition 4.11 and Corollary 4.12 from the semicompact to the general case.

Theorem 6.1. (i) We have a schematic equality

$$
\mathbb{D}_{2}\left({ }_{\mathfrak{a}} \phi\right)=\mathcal{H} \mathcal{E}_{B}^{\{2\}} \cup \mathrm{R}^{\mathrm{sep}}(X / B) .
$$

Moreover the fibre of $\mathcal{H E}_{B}^{\{2\}}$ corresponding to a boundary fibre $X_{0}$ of $X / B$ is empty if $X_{0}$ is not hyperelliptic; if $X_{0}$ is hyperelliptic, the fibre consists of the locus of hyperelliptic schemes contained in some 2-component, each endowed with the collection of hyperelliptic middle azimuths on $\mathrm{X}_{0}$.

(ii) For each fibre $X_{0}$ and each azimuthal subscheme $z$ contained in a 2-component of $X_{0}$ but not supported at a node, the image of a $\phi(z)$ coincides with that of the Brill-Noether map associated to the sepcanonical system on $X_{0}$ compatible with $z$.

Proof. We begin with the observation that both assertions are local on $X_{B}^{\{2\}}$; the second can be checked fibre by fibre, though the second does involve scheme structure over $B$. We follow the broad outline of the proof of Theorem 7.5 of [18]. We will prove both assertions using simultaneous induction on the number of components of a fibre $X_{0}$.

Next, note that if the fibre $X_{0}$ is hyperelliptic, it is of semicompact type and then our assertions already follow from the results of Part 1, specifically Proposition 4.11 and Corollary 4.12. Therefore, we may henceforth assume that $X_{0}$ is non-hyperelliptic.

We will focus next on proving that if $X_{0}$ is non-hyperelliptic as 'plain' curve, ignoring any azimuthal data, then, over a neighborhood of the corresponding point $0 \in B$, the degeneracy scheme $\mathbb{D}_{2}\left({ }_{a} \phi\right)$ is contained in $\mathrm{R}^{\text {sep }}(X / B)$. The opposite containment is obvious by generalizing to a nearby fibre of compact type with the same set of seps as $X_{0}$ (and no other singularities). By the results of $\S 1$, we may assume $X_{0}$ is 2-separable, i.e. contains a sep or bisep. Suppose we are given an azimuthal scheme $z$ of $X_{0}$. We will show that the azimuthal Brill-Noether image $\operatorname{im}\left({ }_{\mathfrak{a}} \phi(z)\right)$ is 2-dimensional. To this end we will need to distinguish a hierarchy of cases and subcases. We suppose next that there exists a $\operatorname{sep}$ or bisep $\theta$, so that $z$ is left of $\theta$ and, for now, 
also that $z$ (more precisely, the underlying scheme) is disjoint from $\theta$. Let ${ }_{\mathrm{L}} D^{\dagger}={ }_{\mathrm{L}} X_{\partial_{\theta}}^{\{2\}}$ be the associated divisor, containing all azimuthal schemes left of $\theta$. Let $\mathbb{E}^{\prime} \subset{ }_{\mathfrak{a}} \mathbb{E}$ be the subsheaf corresponding to all the echelon modifications except at $\theta$. Let $\mathbb{E}^{\prime, 0}$ denote the bundle obtained by applying similar modifications on $\mathbb{E}^{\cdot, 0}$. Then we get a comparison diagram analogous to 3.2.1

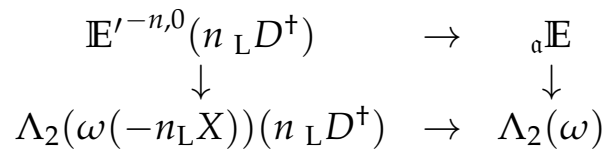

where $n=2$ (sep case) or $n=1$ (bisep case). The left column restricts, up to a twist, to the azimuthal Brill-Noether mapping of ${ }_{\mathrm{L}} X$.

Because $X_{0}$ is non-hyperelliptic, at least one of the pairs $\left({ }_{\mathrm{L}} X(\theta),{ }_{\mathrm{L}} \theta\right),\left({ }_{\mathrm{R}} X(\theta),{ }_{\mathrm{R}} \theta\right)$ is non- hyperelliptic. We first analyze the former case (the two are distinct in that ${ }_{\mathrm{L}} X(\theta)$ is the side containing $z$ ). Within this case, the worst-case scenario is that ${ }_{\mathrm{L}} X(\theta)$ (as plain curve) and $\left({ }_{\mathrm{R}} X(\theta),{ }_{\mathrm{R}} \theta\right)$ (as pair) are hyperelliptic. In particular, ${ }_{\mathrm{L}} X(\theta)$ and ${ }_{\mathrm{R}} X(\theta)$ are of semicompact type. Then, because the pair $\left({ }_{L} X(\theta),{ }_{L} \theta\right)$ is non-hyperelliptic , either ${ }_{L} \theta$ (bisep case) or $2{ }_{L} \theta$ (sep case) is a (degree-2) nonhyperelliptic divisor. If $z$ is not contained in any single 2-component of ${ }_{\mathrm{L}} X(\theta)$, it is easy to see that the canonical system of ${ }_{\mathrm{L}} X(\theta)$ already separates $z$, hence ${ }_{a} \phi$ is surjective over $z$ (this is independent of any azimuthal data). Thus we may assume $z$ is contained in a 2-component $Y$ of ${ }_{\mathrm{L}} X(\theta)$. If $Y$ is the 'right-extremal' 2 -component, i.e. the one that contains ${ }_{\mathrm{L}} \theta$, then by the above comparison diagram, the azimuthal Brill-Noether image $\operatorname{im}\left({ }_{a} \phi(z)\right)$ contains the Brill-Noether image of $\omega_{Y}\left({ }_{L} \theta\right)$ (bisep case) or $\omega_{Y}\left({ }_{\mathrm{L}} 2 \theta\right)$ over $z$, which is already onto by Lemma 3.8 of [18]. If $z$ is not contained in the extremal 2-component, then a 2-component $Y$ containing $z$ contains ${ }_{\mathrm{L}} \theta^{\prime}$ for some sep or bisep of ${ }_{\mathrm{L}} X(\theta)$ (necessarily $\neq \theta$ ), so that ${ }_{\mathrm{R}} X\left(\theta^{\prime}\right)$ is non- hyperelliptic and then, again by using the comparison diagram, the Brill-Noether image over $z$ contains that of $\omega_{Y}\left(3_{\mathrm{L}} \theta^{\prime}\right)$ (sep case) or $\omega_{Y}\left(2_{\mathrm{L}} \theta^{\prime}\right)$ which is onto by Lemma 3.1 of [18]. This concludes the case where $z$ is contained in a non-hyperelliptic side of $\theta$.

At this point, we may assume that for any sep or maximal bisep $\theta$ having $z$ on one side, say left, the pair $\left({ }_{\mathrm{L}} X(\theta),{ }_{\mathrm{L}} \theta\right)$ is hyperelliptic. Suppose such $\theta$ actually exists. Then if $z$ is not contained in any 2-component of ${ }_{\mathrm{L}} X(\theta)$, we can conclude as above. Else, our assumption implies that $z$ must be contained in a left-extremal 2-component $Y$, of ${ }_{\mathrm{L}} X(\theta)$, i.e. on that has the form $Y={ }_{\mathrm{L}} X\left(\theta^{\prime}\right)$ for some sep or bisep $\theta^{\prime}$ (possibly $=\theta$ ) (otherwise, $z \subset Y \subset{ }_{\mathrm{R}} X\left(\theta^{\prime \prime}\right)$ for some $\theta^{\prime \prime}$ and ${ }_{\mathrm{R}} X\left(\theta^{\prime \prime}\right)$ cannot be hyperelliptic). Therefore, we may as well assume $Y={ }_{\mathrm{L}} X(\theta)$. Thus $(Y, \theta)$ is 2-inseparable hyperelliptic, i.e. either $\theta$ (bisep case) or $2 \theta$ (sep case) is a member of the $g_{2}^{1}$. Then by Lemma 3.1 of [18], the system $\left|\omega_{Y}\left(3_{L} \theta\right)\right|$ (sep case) or $\left|\omega_{Y}\left(2_{L} \theta\right)\right|$ (bisep case) is very ample, therefore the left column of (6.3.2) is surjective over ${ }_{\mathrm{L}} D^{\dagger}$. Therefore ${ }_{\mathrm{a}} \phi$ is surjective over the interior of ${ }_{\mathrm{L}} D^{\dagger}$ and hence over $z$.

It remains to consider the case where $z$ is not on one side of any sep or maximal bisep. This implies $z=\left(z_{1}, z_{2}\right), z_{i} \in Y_{i}$ where $Y_{1}, Y_{2}$ are extremal vertices in the 2-separation tree $G_{2}\left(X_{0}\right)$. But then because $g\left(Y_{i}\right)>0, z_{1}, z_{2}$ are already separated by the canonical system of $X_{0}$, and in particular ${ }_{a} \phi(z)$ is surjective.

This completes that the proof that if $X_{0}$ is non-hyperelliptic then assertion (ii) holds for $X_{0}$ and the degeneracy locus of ${ }_{a} \phi$ on $\left(X_{0}\right)^{\{2\}}$ is supported on, hence coincides with, $R^{\operatorname{sep}}\left(X_{0}\right)$. Then a computation as in 3.3 (which is valid locally over $B$ ) shows that for $X_{0}$ non-hyperelliptic, 
the locus $\mathbb{D}_{2}\left({ }_{\mathfrak{a}} \phi\right)$ coincides schematically with $\mathrm{R}^{\mathrm{sep}}(X / B)$ over a neighborhood of $0 \in B$. This completes the proof of Theorem 6.1.

Now a dimension count similar to the proof of Proposition 3.5 shows the following result, which also follows from the Harris-Mumford theory of admissibe covers:

Corollary 6.2. A stable curve is hyperelliptic if and only if it is the limit of smooth hyperelliptic curves.

Corollary 6.3. Notations as above, assume $X / B$ admits a section s disjoint from the singular locus of $X$, and let $Y_{s} \subset X_{B}^{\{2\}}$ be the inverse image of the locus of schemes meeting $s(B)$. Then

$$
\left[\mathbb{D}_{2}\left({ }_{\mathfrak{a}} \phi\right)\right] \cap\left[Y_{S}\right]=\left[\mathcal{H} \mathcal{E}_{B}^{\{2\}}\right] \cap\left[Y_{S}\right]
$$

Proof. Immediate from the fact that $s(B)$ is disjoint from the singular locus of $X / B$, i.e. the locus of singular points of fibres, hence $Y_{S}$ is disjoint from $R^{\operatorname{sep}}(X / B)$.

Various sheaves associated with degeneracy loci admit resolutions by Eagon-Northcott-type complexes (see [11], I.B.2 or [2]). The simplest of these yields

Corollary 6.4. We have, modulo $R^{\operatorname{sep}}(X / B)$ :

$$
\left[\mathcal{O}_{\mathcal{H} \mathcal{E}_{B}^{\{2\}}}\right]=c\left({ }_{\mathfrak{a}} \mathbb{E} \otimes \Lambda_{2}(\omega)^{*}\right) / c\left(\operatorname{Sym}^{2}\left({ }_{\mathfrak{a}} \mathbb{E}\right) \otimes \operatorname{det}\left(\Lambda_{2}(\omega)\right)^{*}\right) .
$$

\section{EXCESS DEGENERACY AND FUNDAMENTAL CLASS}

7.1. Excess Porteous. Motivated by Theorem 6.1, our purpose here is to analyze intersectiontheoretically a simple excess-degeneracy situation, aiming for a Porteous-type formula.

More explcitly, let

$$
\phi: E \rightarrow F
$$

be a map of vector bundles of respective ranks $g, 2$ on a variety $X$, and denote by $\mathbb{D}_{2}(\phi)$ its degeneracy scheme, locally defined by the $2 \times 2$ minors. Let $Z$ be a sum of irreducible components of $\mathbb{D}_{2}(\phi)$, purely of (possibly excessive) dimension $\ell \geq g-1$. The 'expected' or virtual class of the degeneracy locus is computed by Porteous's formula ( [7], Thm. 14.4 and Example 14.4.9):

$$
\left[\mathbb{D}_{2}(\phi)\right]_{\text {vir }}=(-1)^{g-1}[c(E) / c(F)]_{g-1} .
$$

We are interested in the contribution of $Z$ to the virtual degeneracy locus of $\phi$, i.e. the virtual fundamental class of $Z,[Z]_{\text {vir. }}$. Were the rank of $F$ equal to 1 rather than 2, i.e. were the degeneracy locus a zero locus, this class would be computed directly by the 'local' intersection theory of Fulton-MacPherson [7]. Here we will spell out a simple extension, implicit in [7], to the case where $F$ has rank 2.

Assume

(i) The rank of $\phi$ is at least 1 everywhere.

(ii) Off a subset $Y \subset Z$ of codimension $g$ in $X, Z$ is regular, i.e. a local complete intersection of codimension $\ell$ in $X$.

Since we are interested in codimension $g-1$ or less, $Y$ can be ignored, i.e. we can replace $X$ by $X \backslash Y$ and assume $Y=\varnothing$. Set

$$
K=\operatorname{ker}\left(E_{Z} \rightarrow F_{Z}\right), C=\operatorname{coker}\left(E_{Z} \rightarrow F_{Z}\right), N_{Z}=\operatorname{Hom}\left(I_{Z}, \mathcal{O}_{Z}\right)
$$


where $E_{Z}=\left.E\right|_{Z}, F_{Z}=\left.F\right|_{Z}$. As $Y=\varnothing$, these are Z-locally free of ranks $g-1,1, \ell$, respectively. Let

$$
z=[Z]_{\mathrm{vir}}=\left[\frac{c\left(K^{*} \otimes C\right)}{c\left(N_{Z}\right)}\right]_{g-1-\ell}
$$

Proposition 7.1. Assumptions and notations as above, the contribution of $Z$ to the class of the degeneracy locus of $\phi$ is $z$. In particular, if $\mathbb{D}_{2}(\phi)=U \cup Z$ with $U$ of codimension $g-1$ and $Z \cap U$ purely of codimension $\geq g$, then $[U]+z$ is the virtual degeneracy class given by Porteous's formula (7.1.1).

Proof. In the case of a zero-locus in place of degeneracy locus, the analogous formula is given in [7]. To extend to the degeneracy situation, we pass as usual to the projective bundle $\mathbb{P}(F)$ (of rank-1 quotients (!), unlike in [7]) with its Grothendieck quotient $\pi^{*} F \rightarrow \mathcal{O}(1)$. There we have a composite map

$$
\pi^{*} E \rightarrow \mathcal{O}(1)
$$

whose zero-locus contains a component $\tilde{Z}$ projecting isomorphically to $Z$. By the excess zero-set formula of Fulton-MacPherson, the contribution of $\tilde{Z}$ to the zero-set is

$$
\left[\frac{c\left(E^{*}(1)\right)}{c\left(N_{\tilde{Z}}\right)}\right]_{g-1-\ell} \text {. }
$$

Now by a reult of Golubitsky and Guillemin (computation of normal bundles to degeneracy loci, [8], p. 145, recounted in [10]), the latter corresponds to (7.1.2) under the projection $\tilde{Z} \rightarrow Z$. Indeed the difference between $E^{*}(1)$ and $K^{*} \otimes C$ is precisely the 'vertical' subsheaf, that is also the difference between $N_{\tilde{Z}}$ and $N_{Z}$, and these cancel out.

7.2. Fundamental class of the hyperelliptics. We aim to apply Proposition 7.1 with $Z=R^{\operatorname{sep}}(X / B)=$ $\sum_{\theta} R_{\theta}$. In light of Theorem 6.1, this will compute the class of the extended hyperelliptic locus in $X_{B}^{\{2\}}$.

We will evaluate (7.1.2) to obtain the contribution of $R_{\theta}$ for a fixed $\theta$ to the degeneracy locus of the modified Brill-Noether $\phi_{\theta}$. Because the $R_{\theta}$ are disjoint for different $\theta$, the total contribution of $\mathrm{R}^{\mathrm{sep}}(X / B)$ will be the sum of those. The ultimate result is (7.2.7). The reader only interested in the hyperelliptic class on the base $B$ is in for some good news: (s)he may skip ahead to Corollary 7.3 which shows that the contribution of $R^{\text {sep }}$ to the latter vanishes for trivial reasons.

To begin with, it is clear, e.g. from (3.3.5), that the image of $\phi_{\theta}$ at each point $z \in R_{\theta}$, i.e. a length-2 scheme with ideal $I_{z}$ co-supported on $\theta$, coincides with the 1-dimensional space of sections of $\omega \otimes \mathcal{O}_{z}$ vanishing on the length-1 subscheme $\theta \subset z$, i.e. the quotient

$$
\omega \otimes I_{\theta} / \omega \otimes I_{z}=\omega \otimes\left(I_{\theta} / I_{\theta}^{2}\right) / \omega \otimes\left(I_{z} / I_{\theta}^{2}\right) \text {. }
$$

Clearly, further echelon modifications of $\mathbb{E}_{\theta}$ corresponding to other sep/biseps don't change that image, so the same is true for ${ }_{a} \phi$ in place of $\phi_{\theta}$. Because $\left.\omega\right|_{\theta}=\mathcal{O}_{\theta}$ by residues and $I_{\theta} / I_{\theta}^{2}=$ ${ }_{\mathrm{L}} \psi \oplus{ }_{\mathrm{R}} \psi$, this image sheaf coincides with $\mathcal{O}_{R_{\theta}}(1)$ under the identification

$$
R_{\theta}=\mathbb{P}\left({ }_{\mathrm{L}} \psi \oplus{ }_{\mathrm{R}} \psi\right) \text {. }
$$

Because $\mathcal{O}_{R_{\theta}}(1)=\operatorname{det}\left(\Lambda_{2}(\omega)\right) \otimes \mathcal{O}_{R_{\theta}}$, it follows that the cokernel of $\left.{ }_{a} \phi\right|_{R_{\theta}}$ is the trivial bundle. Thus, setting $h_{\theta}=c_{1}\left(\mathcal{O}_{R_{\theta}}(1)\right)$, we have, with the notations of (7.1.2),

$$
C=\mathcal{O}, K \sim\left[{ }_{a} \mathbb{E}\right]-h_{\theta},
$$


therefore the numerator of (7.1.2) evaluates to

$$
c\left(K^{*} \otimes C\right)=\frac{c\left({ }_{\mathfrak{a}} \mathbb{E}_{\theta}^{*}\right)}{1-h_{\theta}} .
$$

As for the denominator, note first that we can write

$$
X_{\partial_{\theta}}^{\{2\}}={ }_{\mathrm{L}} X_{\partial_{\theta}}^{\{2\}} \cup{ }_{\mathrm{R}} X_{\partial_{\theta}}^{\{2\}} \cup X_{12}
$$

where $X_{12}$ is the inverse image of ${ }_{L} X \times_{\partial_{\theta}}{ }_{\mathrm{R}} X$ and can be identified with the blowup of the latter in $\left({ }_{\mathrm{L}} \theta,{ }_{\mathrm{R}} \theta\right)$ with exceptional divisor $R_{\theta}$. We have

$$
N_{R_{\theta} / X_{12}}=\mathcal{O}_{R_{\theta}}(-1) \text {. }
$$

On the other hand, writing ${ }_{*} \theta^{[2]}$ for the locus of subschemes of ${ }_{*} X^{[2]}$ supported on $* \theta, *={ }_{L}, \mathrm{R}$, we have

$$
\begin{aligned}
\left.N_{X_{12} / X_{B}\{2\}}\right|_{R_{\theta}}=\mathcal{O}_{R_{\theta}}\left(\partial_{\theta}-{ }_{\mathrm{L}} X_{\partial_{\theta}}^{\{2\}}-\right. & \left.{ }_{\mathrm{R}} X_{\partial_{\theta}}^{\{2\}}\right)=\mathcal{O}_{R_{\theta}}\left(\partial_{\theta}-{ }_{\mathrm{L}} \theta^{[2]}-{ }_{\mathrm{R}} \theta^{[2]}\right) \\
& =\mathcal{O}_{R_{\theta}}\left(-\psi_{1}-\psi_{1}-{ }_{\mathrm{L}} \theta^{[2]}-{ }_{\mathrm{R}} \theta^{[2]}\right)
\end{aligned}
$$

where the latter results from a formula we have used before, due to Faber [5], §2, which says, in our notations, that

$$
\left.\partial_{\theta}\right|_{\theta}=-\psi_{1}-\psi_{2}
$$

Note that ${ }_{*} \theta \cdot R_{\theta}$ can be identified with the section $\mathbb{P}\left({ }_{*} \psi_{i}\right)$ on $\mathbb{P}\left({ }_{\mathrm{L}} \psi \oplus{ }_{\mathrm{R}} \psi\right)=R_{\theta}$. Now on $R_{\theta}$, we have

$$
{ }_{\mathrm{L}} \psi+{ }_{\mathrm{R}} \theta^{[2]} \sim{ }_{\mathrm{R}} \psi+{ }_{\mathrm{L}} \theta^{[2]} \sim h_{\theta}
$$

Therefore we have

Therefore

$$
c_{1}\left(\left.N_{X_{12} / X_{B}^{\{2\}}}\right|_{R_{\theta}}\right)=-2 h_{\theta}
$$

All in all, we get for the contribution of $R_{\theta}$ :

$$
\left[R_{\theta}\right]_{\text {vir }}=\left[\frac{\left.c\left({ }_{a} \mathbb{E}^{*}\right)\right|_{R_{\theta}}}{\left(1-h_{\theta}\right)^{2}\left(1-2 h_{\theta}\right)}\right]_{g-3} .
$$

It remains to compute the numerator. To this end we now work over $R_{\theta}$. Note that the restriction of $\mathbb{E}$ on the boundary $\partial_{\theta}$ (a fortiori, on $R_{\theta}$ ) splits as ${ }_{L} \mathbb{E} \oplus{ }_{\mathrm{R}} \mathbb{E}$, where each ${ }_{*} \mathbb{E}$ is the Hodge bundle of ${ }_{*} X / \delta_{\theta}$, and ${ }_{*} \mathbb{E}$ admits an exact sequence induced by restriction of differentials on $* \theta$ :

$$
0 \rightarrow_{*} \mathbb{E}^{-1} \rightarrow_{*} \mathbb{E} \rightarrow_{*} \psi \rightarrow 0,_{*}=\mathrm{L}, \mathrm{R} .
$$

Thus ${ }_{*} \mathbb{E}^{-1}$ is the rank- $\left(g\left({ }_{*} X\right)-1\right)$ bundle of relative differentials of ${ }_{*} X / \delta_{\theta}$ vanishing on ${ }_{*} \theta$ (it is the bundle denoted $\mathbb{E}^{0,-1}$ or $\mathbb{E}^{-1,0}$ in $\$ 3.1$ ) and ${ }_{*} \psi$ is the usual line bundle on $\delta_{\theta}$ (and also by pullback, on $R_{\theta}$ ). The saturated image $\mathfrak{a}_{\mathfrak{a} *} \mathbb{E}$ of ${ }_{*} \mathbb{E}$ in $\left.{ }_{\mathfrak{a}} \mathbb{E}\right|_{\delta_{\theta}}$ fits in an exact sequence

$$
0 \rightarrow_{\mathfrak{a} *} \mathbb{E}^{-1}\left(2_{+} D\right) \rightarrow_{\mathfrak{a} *} \mathbb{E} \rightarrow_{*} \psi \otimes \mathcal{O}\left({ }_{+} D\right) \rightarrow 0
$$

where $\dagger$ is the opposite of $*$, i.e $\dagger={ }_{\mathrm{L}}$ if and only if $*={ }_{\mathrm{R}}$ and ${ }_{\mathrm{a} *} \mathbb{E}^{-1}$ is the appropriate subbundle of ${ }_{\mathfrak{a}} \mathbb{E}\left({ }_{*} X / \partial_{\theta}\right)$, i.e. the azimuthal modification of the Hodge bundle of the family ${ }_{*} X / \partial_{\theta}$, namely 
the subbundle defined by vanishing on ${ }_{*} \theta$ analogously to ${ }_{*} \mathbb{E}^{-1}$ above; thus as virtual bundles, we have

$$
\left[a * \mathbb{E}^{-1}\right]=\left[{ }_{a} \mathbb{E}\left({ }_{*} X / \partial_{\theta}\right)\right]-{ }_{*} \psi
$$

in which $* \psi$ is viewed via pullback from the base as a line bundle on $\left(X_{i}\right)_{\delta_{\theta}}^{\{2\}}$. Moreover, clearly

$$
{ }_{*} D \cdot R_{\theta}={ }_{*} \theta^{[2]} \text {. }
$$

Putting it all together, we obtain

$$
c\left(\left.{ }_{\mathfrak{a}} \mathbb{E}^{*}\right|_{R_{\theta}}\right)=\frac{c\left({ }_{\mathfrak{a ~ L}} \mathbb{E}^{*}\left(-2{ }_{\mathrm{L}} \theta^{[2]}\right)\right) \cdot\left(1-{ }_{\mathrm{L}} \psi-{ }_{\mathrm{R}} \theta^{[2]}\right)}{1-{ }_{\mathrm{L}} \psi-2{ }_{\mathrm{R}} \theta^{[2]}} \frac{c\left({ }_{\mathrm{a} \mathrm{R}} \mathbb{E}^{*}\left(-2{ }_{\mathrm{R}} \theta^{[2]}\right)\right) \cdot\left(1-{ }_{\mathrm{R}} \psi-{ }_{\mathrm{L}} \theta^{[2]}\right)}{1-{ }_{\mathrm{R}} \psi-2_{\mathrm{L}} \theta^{[2]}} \cap\left[R_{\theta}\right] .
$$

Now in view of (7.2.2) and (7.2.3), we obtain

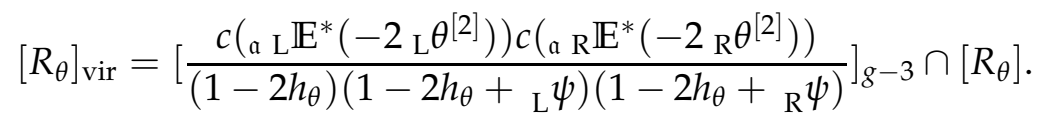

Since for different $\theta, R_{\theta}$ are disjoint, it follows that

$$
\left[\mathrm{R}^{\mathrm{sep}}\right]_{\mathrm{vir}}=\sum_{\theta}\left[\frac{c\left({ }_{\mathrm{a}} \mathbb{E}\left({ }_{\mathrm{L}} X(\theta)\right)^{*}\left(-2{ }_{\mathrm{L}} \theta\right)\right) c\left({ }_{\mathrm{a}} \mathbb{E}\left({ }_{\mathrm{R}} X(\theta)\right)^{*}\left(-2{ }_{\mathrm{R}} \theta\right)\right)}{\left(1-2 h_{\theta}\right)\left(1-2 h_{\theta}+{ }_{\mathrm{L}} \psi_{\theta}\right)\left(1-2 h_{\theta}+{ }_{\mathrm{R}} \psi_{\theta}\right)}\right]_{g-3} \cap\left[R_{\theta}\right]
$$

where we recall that on $R_{\theta}$, we have

$$
h_{\theta}={ }_{\mathrm{L}} \psi_{\theta}+{ }_{\mathrm{R}} \theta^{[2]}={ }_{\mathrm{R}} \psi_{\theta}+{ }_{\mathrm{L}} \theta^{[2]} .
$$

Thus we have finally obtained our main result on the hyperelliptic class:

Theorem 7.2. The fundamental class on $X_{B}^{\{2\}}$ of the closure of the locus of hyperelliptic divisors on smooth hyperelliptic curves is give by

$$
\left.\left[\mathcal{H} \mathcal{E}^{2}\right]=(-1)^{g-1}\left[c{ }_{\mathfrak{a}} \mathbb{E}\right) / c\left(\Lambda_{2}(\omega)\right)\right]_{g-1}-\left[\mathrm{R}^{\mathrm{sep}}\right]_{\mathrm{vir}}
$$

where $\left[\mathrm{R}^{\mathrm{sep}}\right]_{\mathrm{vir}}$ is given by (7.2.7).

As mentioned in the Introduction, the image of this class on $B$ is fully computed in genus 3 , e.g. in [17], $\S 4.5$. For the computation of the boundary in genus 4 , see 8.2 . The computation of this image can be approached via the following

Corollary 7.3. If the image on $B$ of the locus of smooth hyperelliptic fibres of $X / B$ is of codimension $g-2$, then the fundamental class of its closure is, where $\omega:=\omega_{X / B}$ :

$$
[\mathcal{H E}]_{B}=\left(\pi_{B}\right)_{*}\left(\frac{1}{2 g-2}(-1)^{g-1}\left[c\left({ }_{\mathfrak{a}} \mathbb{E}\right) / c\left(\Lambda_{2}(\omega)\right)\right]_{g-1}(\omega)^{[2]}\right) .
$$

Proof. Each smooth hyperelliptic curve contributes a linear pencil to the locus $\mathcal{H E}$ above, and $2 g-2$ many members of this pencil will have a point in common with a fixed canonical divisor. Therefore the formula follows from (7.2.8) by multiplying by $\omega^{[2]}$, except for the vanishing of the term coming from $R^{\text {sep }}$. This term vanishes because, as is well known from residues, $\left.\omega\right|_{\theta}$ is trivial for any node $\theta$, therefore $\omega^{[2]}$ is trivial on $\mathrm{R}^{\theta}$. 


\section{AZIMUTHAL INTERSECTION THEORY}

8.1. Chern classes of azimuthal bundles. We will assume WLOSG 2 that $g>2$. The azimuthal bundle ${ }_{a} \mathbb{E}$ is obtained by a polyechelon modification ( [16], $\left.\S 3\right)$, i.e. sequence of mutually transverse echelon modifications corresponding to seps and biseps, and to compute its Chern classes it suffices to compute how these classes change on passing from one bundle $E$, a partial azimuthal modification, to a further partial azimuthal modification, viz. $E_{\theta}$ or $E_{\underline{\theta}}$. Note that $E$ inherits from the initial $\mathbb{E}$ subsheaves $E \%$ and quotients ${ }_{\mathrm{L}} E,{ }_{\mathrm{R}} E$, which are corresponding modifications of the analogous sheaves associated to $\mathbb{E}$.

8.1.1. Case of sep. We begin with the case of a sep. Thus, we fix a sep $\theta$ and compute the Chern classes of the echelon modification $E_{\theta}$ (see $\$ 3.1$ ). We assume to start with that ${ }_{\mathrm{L}} g{ }{ }_{\mathrm{R}} g>1$. Then the echelon modification is obtained in 2 steps along each of the divisors ${ }_{\mathrm{L}} D,{ }_{\mathrm{R}} D$, which are mutually disjoint. Therefore it will suffice to work out the left modification. This takes the form

$$
E \subset E_{1} \subset E_{2} \text {. }
$$

As virtual bundle, $E_{1}$ has the form

$$
E_{1} \sim E+{ }_{\mathrm{R}} E \otimes \mathcal{O}_{\mathrm{L} D}\left({ }_{\mathrm{L}} D\right)
$$

(where ${ }_{\mathrm{R}} E$ is, in general, the appropriate azimuthal modification of $\pi_{*}\left(\omega_{\mathrm{R}} X / \partial_{\theta}\right)$ with respect to other seps/biseps; in case $E$ is the (unmodified) Hodge bundle itself, ${ }_{\mathrm{R}} E=\pi_{*}\left(\omega_{\mathrm{R}} X / \partial_{\theta}\right)$. The difference is formal difference as virtual bundle). Similarly,

$$
E_{2} \sim E_{1}+{ }_{\mathrm{R}} E^{0} \otimes \mathcal{O}_{\mathrm{L} D}\left(2_{\mathrm{L}} D\right)
$$

where ${ }_{\mathrm{R}} E^{0}$ is an appropriate azimuthal modification of $\pi_{*}\left(\omega_{\mathrm{R}} X / \partial_{\theta}\left(-{ }_{\mathrm{R}} \theta\right)\right)$ and equal to the latter push-forward when $E$ is the Hodge bundle. Thus, when $E$ is the Hodge bundle, then as virtual bundle

$$
{ }_{\mathrm{R}} E^{0}={ }_{\mathrm{R}} E-{ }_{\mathrm{R}} \psi
$$

In particular, ${ }_{\mathrm{R}} E=0$ if ${ }_{\mathrm{R}} g=1$.

Therefore, since ${ }_{\mathrm{L}} D$ and ${ }_{\mathrm{R}} D$ are disjoint, we have in all,

$$
\begin{aligned}
E_{\theta} \sim & E+\left({ }_{\mathrm{R}} E \otimes \mathcal{O}_{\mathrm{L}} D\left({ }_{\mathrm{L}} D\right)+\left({ }_{\mathrm{R}} E-{ }_{\mathrm{R}} \psi\right) \otimes \mathcal{O}_{\mathrm{L}} D\left(2_{\mathrm{L}} D\right)\right) \\
& + \text { mirror }(.),
\end{aligned}
$$

where by convention the 'mirror' of an expression is the corresponding one with each ' $\mathrm{L}^{\prime}$ replaced by ' $\mathrm{R}^{\prime \prime}$ and vice versa. We recall from Lemma 3.3 that $\mathcal{O}_{\mathrm{L}} D\left({ }_{\mathrm{L}} D\right)=\psi^{-1} \otimes \mathcal{O}_{\mathrm{L}} D\left(-{ }_{\mathrm{L}} \theta^{[2]}\right)$. We will use 00.1 and its notations, e.g. (0.1.3). Thus set

$$
\begin{aligned}
{ }_{\mathrm{L}} q= & Q\left({ }_{\mathrm{L}} D,{ }_{\mathrm{R}} E\right) c\left({ }_{\mathrm{R}} E^{\vee}\right),{ }_{\mathrm{R}} q=Q\left({ }_{\mathrm{R}} D,{ }_{\mathrm{L}} E\right) c\left({ }_{\mathrm{L}} E^{\vee}\right) \\
{ }_{\mathrm{L}} q= & e+\left(\begin{array}{l}
e \\
2
\end{array}\right) D-c_{1}+\left(\begin{array}{l}
e \\
3
\end{array}\right) D^{2}-(e-1) D c_{1}-(e-1) c_{1}^{2}+2(e-1) c_{2} \\
& +\left(\begin{array}{l}
e \\
4
\end{array}\right) D^{3}-\left(\begin{array}{c}
e-1 \\
2
\end{array}\right) D^{2} c_{1}-\left(\begin{array}{c}
e-1 \\
2
\end{array}\right) D c_{1}^{2}+c_{1} c_{2}+(e-4) c_{3}
\end{aligned}
$$

where $e={ }_{\mathrm{R}} g, c_{i}=c_{i}\left({ }_{\mathrm{R}} E\right)$ and likewise for the mirror; of course, ${ }_{\mathrm{L}} q,_{\mathrm{R}} q$ depend on $E, \theta$ as well.

\footnotetext{
${ }^{2}$ without loss of significant generality
} 
Then when $E$ is the unmodified Hodge bundle, we have $c\left(i_{\mathrm{L}} D *\left({ }_{\mathrm{R}} E\left({ }_{\mathrm{L}} D\right)\right)\right)=1+i_{\mathrm{L}} D *\left({ }_{\mathrm{L}} q\right)$.

The Chern class of $i_{\mathrm{L}} D *\left({ }_{\mathrm{R}} E^{0}\left({ }_{\mathrm{L}} D\right)\right.$ can be computed using the general Riemann-Roch without denominators directly, but, with a view to an application in genus 4, we shall do so explicitly only for $\mathrm{R}^{g}=2,3$. We shall omit all terms which have codimension $>2$ in the boundary or whose base image has codimension $>1$ in the boundary. Because the class in question will ultimately be multiplied (see (8.2.20) ) by $\omega_{1}$, which has trivial restriction on nodes, we may also omit classes supported on nodes. Now note that $\left({ }_{L} \theta^{(2)}\right)^{2}$ is the sum of a node-supported class (locus of schemes contained in ${ }_{L} \theta$ ) and $-{ }_{L} \psi_{L} \theta^{(2)}$. Therefore, modulo negligible classes, we have

$$
\left({ }_{\mathrm{L}} \theta^{(2)}\right)^{2} \sim-{ }_{\mathrm{L}} \psi_{\mathrm{L}} \theta^{(2)}
$$

Set ${ }_{\mathrm{R}} F={ }_{\mathrm{R}} E^{0} \otimes \mathcal{O}_{\mathrm{L}_{\mathrm{L}}} \sim\left({ }_{\mathrm{R}} E-{ }_{\mathrm{R}} \psi\right)_{\mathrm{L}} D, D={ }_{\mathrm{L}} D$. Then

$$
\begin{aligned}
& \frac{1}{c\left({ }_{\mathrm{R}} F(D)\right)} \sim 1-\left({ }_{\mathrm{R}} \lambda_{-}{ }_{\mathrm{R}} \psi-\left({ }_{\mathrm{R}} g-1\right)\left(\psi+{ }_{\mathrm{L}} \theta^{(2)}\right)\right. \\
& +\left({ }_{\mathrm{R}} \lambda-{ }_{\mathrm{R}} \psi-\left({ }_{\mathrm{R}} g-1\right)\left(\psi+{ }_{\mathrm{L}} \theta^{(2)}\right)\right)^{2}+\left({ }_{\mathrm{R}} g-1\right)\left({ }_{\mathrm{R}} \lambda-{ }_{\mathrm{R}} \psi\right)\left(\psi+{ }_{\mathrm{L}} \theta^{(2)}\right)-\left(\begin{array}{c}
\mathrm{R} g-1 \\
2
\end{array}\right)\left(\psi+{ }_{\mathrm{L}} \theta^{(2)}\right)^{2} \\
& \sim_{\left({ }_{\mathrm{R}} g=2\right)} 1+\left(-{ }_{\mathrm{R}} \lambda+{ }_{\mathrm{R}} \psi+\psi+\theta\right)-2{ }_{\mathrm{L}} \theta^{(2)}\left({ }_{\mathrm{R}} \lambda-{ }_{\mathrm{R}} \psi-\psi\right) \\
& \sim_{\left.{ }_{\mathrm{R}} g=3\right)} 1-\left({ }_{\mathrm{R}} \lambda-{ }_{\mathrm{R}} \psi+2\left(\psi+{ }_{\mathrm{L}} \theta^{(2)}\right)+5_{\mathrm{L}} \theta^{(2)}\left(-{ }_{\mathrm{R}} \lambda+{ }_{\mathrm{R}} \psi+2 \psi\right) .\right.
\end{aligned}
$$

Set

$$
\begin{aligned}
{ }_{\mathrm{L}} q^{0} & =\frac{Q\left({ }_{\mathrm{L}} D,{ }_{\mathrm{R}} F\right)}{c\left({ }_{\mathrm{R}} F(D)\right)}={ }_{\left.{ }_{\mathrm{R}} g=2\right)} \frac{1}{c\left({ }_{\mathrm{R}} F(D)\right)}, \\
1+{ }_{\mathrm{L}} m(\theta, E) & \left.=\left(1+i_{\mathrm{L} D *}\left({ }_{\mathrm{L}} q\right)\right)\left(1+i_{\mathrm{L}} D+{ }_{\mathrm{L}} q^{0}\right)\right) .
\end{aligned}
$$

Note that, because ${ }_{\mathrm{L}} D \cap{ }_{\mathrm{R}} D=\varnothing$, we have $i_{\mathrm{L} D}(*) i_{\mathrm{R}} D(*)=0$. Also, note the elementary formula, for any divisor $D$,

$$
i_{D *}(x) i_{D *}(y)=i_{D *}(x y D)
$$

(clear if $D$ moves, general case follows by moving lemma; or, it follows from the universal formula $\left.\left(\Delta_{X}\right) \cdot X \times X(D \times D) \sim \Delta_{D} \cdot \mathcal{O}_{D}(D)\right)$. Consequently,

$$
{ }_{\mathrm{L}} m(\theta, E)=i_{\mathrm{L}} D *\left({ }_{\mathrm{L}} q+{ }_{\mathrm{L}} q^{0}-(\psi+\theta)_{\mathrm{L}} q_{\mathrm{L}} q^{0}\right)
$$

Then the above becomes

$$
c\left(E_{\theta}\right)=c(E)\left(1+{ }_{\mathrm{L}} m(\theta, E)\right)\left(1+\mathrm{R}_{\mathrm{R}} m(\theta, E)\right)=c(E)\left(1+{ }_{\mathrm{L}} m(\theta, E)+\mathrm{R}_{\mathrm{R}} m(\theta, E)\right) .
$$

Here $E$ will in general be a partial azimuthal modification of the Hodge bundle $\mathbb{E}$ with respect to some biseps and seps different from $\theta$. For $\theta$ of type $(2,2$,$) , we compute, where E$ is the unmodified Hodge bundle:

$$
{ }_{\mathrm{L}} m(\theta, E)=i_{\mathrm{L} D *}\left(3-2\left({ }_{\mathrm{R}} \lambda+\psi+{ }_{\mathrm{L}} \theta^{(2)}\right)+{ }_{\mathrm{R}} \psi+2{ }_{\mathrm{L}} \theta^{(2)}{ }_{\mathrm{R}} \lambda\right) .
$$


Consequently, we get for the $(2,2)$ sep:

$$
\begin{aligned}
c\left(E_{\theta}\right)=1 & +c_{1}(E) \\
& \left.+3\left({ }_{\mathrm{L}} D+{ }_{\mathrm{R}} D\right)\right)+3 c_{1}(E)\left({ }_{\mathrm{L}} D+{ }_{\mathrm{R}} D\right) \\
& +\left(i_{\mathrm{L}} D *\left(-2\left({ }_{\mathrm{R}} \lambda+\psi+{ }_{\mathrm{L}} \theta^{(2)}\right)+{ }_{\mathrm{R}} \psi\right)+2 i_{\mathrm{L}} D *\left({ }_{\mathrm{L}} \theta^{(2)}{ }_{\mathrm{R}} \lambda\right)\right) \\
& + \text { mirror }(.) .
\end{aligned}
$$

8.1.2. Contribution of node scroll. Next, we study the restriction of $\mathbb{E}_{\theta}$ on various scrolls $R_{\theta^{\prime}} \simeq$ $\mathbb{P}\left({ }_{\mathrm{L}} \psi\left(\theta^{\prime}\right) \oplus_{\mathrm{R}} \psi\left(\theta^{\prime}\right)\right)$, where $\theta^{\prime}$ is some sep of $X / B$, possibly equal to $\theta$. By Corollary 7.3 , this does not affect the class of the hyperelliptic locus in the base. First consider the case $\theta^{\prime}=\theta$. Note that

$$
{ }_{*} D \cdot R_{\theta}=\mathbb{P}\left({ }_{*} \psi\right):={ }_{*} S_{\theta}, *={ }_{\mathrm{L}}, \mathrm{R}
$$

(transverse intersection). Thus ${ }_{*} S_{\theta}$ is a section of the $\mathbb{P}^{1}$-bundle $R_{\theta}$. Therefore restricting $\mathbb{E}_{\theta}$ on $R_{\theta}$ is elementary. If $\theta^{\prime} \neq \theta$, then over each point of $\partial_{\theta^{\prime}} \cap \partial_{\theta}, R_{\theta^{\prime}}$ is contained in the interior of at most one of ${ }_{\mathrm{L}} D,{ }_{\mathrm{R}} D$. In the case of a versal family $X / B$, assuming ${ }_{\mathrm{L}} X(\theta),_{\mathrm{L}} X\left(\theta^{\prime}\right)$ have different genera, $R_{\theta^{\prime}}$ is contained in the interior of precisely one of ${ }_{\mathrm{L}} D,{ }_{\mathrm{R}} D$ and is disjoint from the other. Therefore, if we arrange notations so that each (unoriented) sep $\theta$ appears twice, once with each orientation, we have

$$
\left({ }_{\mathrm{L}} D+{ }_{\mathrm{R}} D\right) \cdot R_{\theta^{\prime}}=\partial_{\theta} \cdot R_{\theta^{\prime}} .
$$

The case of a bisep $\underline{\theta}$ is similar: here we can always assume $\theta^{\prime} \in{ }_{\mathrm{L}} X(\underline{\theta})$ and then $\underline{\theta}$ contributes a modification along $\Xi(\underline{\theta})$. Putting all together, we can write

$$
\left.{ }_{\mathrm{a}} \mathbb{E}\right|_{R_{\theta^{\prime}}}=\left.\left(\mathbb{E}\left({ }_{\mathrm{L}} S_{\theta^{\prime}}+{ }_{\mathrm{R}} S_{\theta^{\prime}}+\sum_{\theta \neq \theta^{\prime}} 2 \partial_{\theta}+\sum_{\theta^{\prime} \in \mathrm{L} X(\underline{\theta})} \Xi(\underline{\theta})\right)+A+B+C\right)\right|_{R_{\theta^{\prime}}}
$$

where

$$
\begin{gathered}
A=\sum_{\substack{\theta \neq \theta^{\prime} \\
\mathrm{L} D(\theta) \cap R_{\theta^{\prime}} \neq \varnothing}}-2_{\mathrm{L}} \mathbb{E}(\theta) \otimes \mathcal{O}_{\partial_{\theta}}\left(2 \partial_{\theta}\right)-{ }_{\mathrm{L}} \mathbb{E}(\theta) \otimes \mathcal{O}_{\partial_{\theta}}\left(\partial_{\theta}\right)-{ }_{\mathrm{L}} \psi_{\theta^{\prime}}^{-1} \otimes \mathcal{O}_{\partial_{\theta}}\left(2 \partial_{\theta}\right) \\
B=-2_{\mathrm{L}} \mathbb{E}\left(\theta^{\prime}\right) \otimes \mathcal{O}_{\mathrm{L}} S_{\theta^{\prime}}\left(2_{\mathrm{L}} S_{\theta^{\prime}}\right)-{ }_{\mathrm{L}} \mathbb{E}\left(\theta^{\prime}\right) \otimes \mathcal{O}_{\mathrm{L}} S_{\theta^{\prime}}\left({ }_{\mathrm{L}} S_{\theta^{\prime}}\right)-{ }_{\mathrm{L}} \psi\left(\theta^{\prime}\right)^{-1} \otimes \mathcal{O}_{\mathrm{L}} S_{\theta^{\prime}}\left(2_{\mathrm{L}} S_{\theta^{\prime}}\right) \\
-2_{\mathrm{R}} \mathbb{E}\left(\theta^{\prime}\right) \otimes \mathcal{O}_{\mathrm{R}} S_{\theta^{\prime}}\left(2_{\mathrm{R}} S_{\theta^{\prime}}\right)-{ }_{\mathrm{R}} \mathbb{E}\left(\theta^{\prime}\right) \otimes \mathcal{O}_{\mathrm{R}} S_{\theta^{\prime}}\left({ }_{\mathrm{R}} S_{\theta^{\prime}}\right)-{ }_{\mathrm{R}} \psi\left(\theta^{\prime}\right)^{-1} \otimes \mathcal{O}_{\mathrm{R}} S_{\theta^{\prime}}\left(2_{\mathrm{R}} S_{\theta^{\prime}}\right) \\
C=-\sum_{\theta^{\prime} \in{ }_{\mathrm{L}} X(\underline{\theta})} \mathrm{L}\left(\underline{\theta^{\prime}}\right)(\mathbb{E}(\underline{\theta})) .
\end{gathered}
$$

Note that the divisors involved, i.e. $\partial_{\theta}$ and $\Xi(\underline{\theta})$ are transverse to $R\left(\theta^{\prime}\right)$.

8.1.3. Case of bisep. The case of the modification corresponding to an oriented proper bisep $\underline{\theta}$ is similar and simpler in the sense that the modification has just one step, i.e. equals $E_{1}$, and there is no residual scheme like $R(\theta)$. Here $D$ is the divisor $\Xi_{\underline{\theta}}$ (see $(5.4 .5)$ ) and we have

$$
E_{\underline{\theta}} \sim E(D)-{ }_{\mathrm{L}} E \otimes \mathcal{O}_{D}(D) \sim E+{ }_{\mathrm{R}} E^{0} \otimes \mathcal{O}_{D}(D)
$$

where $\mathcal{O}_{D}(D)$ is given by (the dual of) (5.4.6) and of course ${ }_{\mathrm{L}} E={ }_{\mathrm{L}} E(\underline{\theta})$, the quotient associated to the oriented bisep $\underline{\theta}$, which is the appropriate echelon modification of $\pi_{*}\left(\omega_{\mathrm{L}} X(\underline{\theta})(\underline{\theta})\right)$, and is 
defined over $\Xi_{\underline{\theta}}$. Of course, at the end of the day when both $\underline{\theta}$ and its opposite are accounted for, (8.1.17) will be replaced by

$$
\begin{aligned}
E\left({ }_{\mathrm{L}} D\right. & \left.+{ }_{\mathrm{R}} D\right)-{ }_{\mathrm{L}} E \otimes \mathcal{O}_{\mathrm{L}} D\left({ }_{\mathrm{L}} D\right)-{ }_{\mathrm{R}} E \otimes \mathcal{O}_{\mathrm{R}} D\left({ }_{\mathrm{R}} D\right) \\
& \sim E+{ }_{\mathrm{R}} E^{0} \otimes \mathcal{O}_{\mathrm{L}} D\left({ }_{\mathrm{L}} D\right)+{ }_{\mathrm{L}} E^{0} \otimes \mathcal{O}_{\mathrm{R}} D\left({ }_{\mathrm{R}} D\right) .
\end{aligned}
$$

See 8.3 on how to compute the terms appearing in (8.1.17).

8.2. Hyperelliptic class in genus 4. In [4], Faber gives a set of 14 generators for the group $R^{2}$ of tautological classes in degree 2 and genus 4 , and shows that these generators satisfy a unique relation, so that $R^{2}$ is of rank 13. In [6], Faber and Pandharipande state a formula for the hyperelliptic class in genus 4 in terms of Faber's generators; their approach is via 'undetermined coefficients', determining the coefficients involved in expressing the hyperelliptic class in terms of Faber's generators by pairing with suitable test classes. To compare their results with ours, we note at the outset that our azimuthal modifications involve only reducible curves and separating (collections of) nodes and consequently, any explicit version of our formula (7.3) will have coefficient zero for the class $\delta_{00}$ of irreducible binodal curves. Therefore, for comparison, we modify the formula of [6], Prop. 5, by adding a suitable multiple (viz. -4/9) of Faber's relation, so as to yield a (uniquely determined) formula with coefficient 0 for $\delta_{00}$, viz.

$$
\begin{aligned}
{[\overline{\mathcal{H E}}]_{4}=} & \frac{1}{6}\left(\kappa_{2}+63 \lambda_{1}^{2}-16 \lambda_{1} \delta_{0}-66 \lambda_{1} \delta_{1}+\delta_{0}^{2}+8 \delta_{0} \delta_{1}+13 \delta_{1}^{2}+27 \delta_{2}^{2}\right. \\
& \left.-2 \gamma_{1}-\frac{1}{3} \delta_{01 a}+4 \delta_{11}+18 \lambda_{1} \delta_{2}+18 \delta_{1} \delta_{2}\right) .
\end{aligned}
$$

Here $\gamma_{1}$ is the class of the locus of curves with a proper bisep of type $(2,1)$, i.e. unions of curves of genera $2,1, \delta_{01 a}$ is the class of the (codimension-2) locus of curves containing a 1-nodal rational tail, and $\delta_{11}$ is the locus of chains of genera 1,2,1, i.e. the curves admitting an improper bisep of type $(1,2,1)$. Note that, in our notation,

$$
\delta_{01 a}=12_{\mathrm{L}} \psi_{\delta_{1}}=12_{\mathrm{L}} \lambda_{\delta_{1}}
$$

Also, $\delta_{11}$ is the (normal crossing) double locus of the $\Delta_{1}$ boundary divisor.

The formula (8.2.19) implies an analogous one (same coefficients) for the hyperelliptic class $[\mathcal{H E}]_{B}$ on the base of any given family of stable curves. Our purpose here is to recover (8.2.19) via azimuthal modifications. In our approach, the generating classes are not presumed but arise out of the construction.

8.2.1. The uncorrected formula. It will be convenient to work in an ordered version of $X_{B}^{\{2\}}$, namely $X_{B}^{\{2\}} \times_{X_{B}^{(2)}} W^{2}(X / B)$ where $W^{*}(X / B)$ is the flag Hilbert scheme (cf. [15]), which dominates $X_{B}^{2}$. We will denote by $\omega_{i}$ the $i$-th pullback $p_{i}^{*}(\omega)$ etc. Then the analogue of Corollary 7.3 reads

$$
[\mathcal{H E}]_{B}=p\left({ }_{a} \mathbb{E}\right):=\frac{-1}{6} \pi_{B *}\left(\left[\frac{c\left({ }_{\mathfrak{a}} \mathbb{E}\right)}{1+\omega_{1}+\omega_{2}-\Gamma^{(2)}+\omega_{1} \omega_{2}-\omega_{1} \Gamma^{(2)}}\right]_{3} \omega_{1}\right) .
$$

We note that

$$
1 / c\left(\Lambda_{2}(\omega)\right)=1-\left(\omega_{1}+\omega_{2}-\Gamma\right)+\omega_{1}^{2}+\omega_{29}^{2}+\omega_{1} \omega_{2}-4 \omega \Gamma+(\text { higher }- \text { order terms }) .
$$


We can naturally decompose $p(E)=\sum_{i=0}^{3} p_{i}(E)$ where

$$
p_{i}(E)=c_{i}(E) q_{3-i}, q_{3-i}:=\omega_{1}\left(\frac{-1}{6 c\left(\Lambda_{2}(\omega)\right)}\right)_{3-i}
$$

$\left(p_{0}(E)=q_{3}\right.$ is of course independent of $E$ ). We note that the analogue of the RHS of (8.2.20) for the unmodified Hodge bundle was essentially computed in [14] and reproduced in [4] and elsewhere, and equals

$$
p(\mathbb{E})=\frac{-1}{6} \pi_{B *}\left(\left[\frac{c(\mathbb{E})}{1+\omega_{1}+\omega_{2}-\Gamma^{(2)}+\omega_{1} \omega_{2}-\omega_{1} \Gamma^{(2)}}\right]_{3} \omega_{1}\right)=\frac{1}{6}\left(\kappa_{2}+63 \lambda_{1}^{2}-16 \lambda_{1} \delta+\delta^{2}\right)
$$

where $\delta$ is the entire boundary and we have used Mumford's formula $\kappa_{1}=12 \lambda_{1}-\delta$. This expression computes the hyperelliptic class correctly in the interior, i.e. on the open set of smooth fibres in $B$ but to be correct everywhere must be augmented by the appropriate boundary contributions, that we proceed to compute via replacing $\mathbb{E}$ by a $\mathbb{E}$. The strategy will be, more or less, to represent

$$
{ }_{\mathfrak{a}} \mathbb{E}=\left(\left(\mathbb{E}_{\underline{\theta}_{2,1}}\right)_{\theta_{2,2}}\right)_{\theta_{1,3}} .
$$

This strategy will require some modification due to the double locus of the $(1,3)$ boundary, denoted $\Delta_{1,1}$ by Faber. Note that, because the boundary locus $\partial_{\underline{\theta}_{2,1}}$ has codimension 2, its azimuthal modification does not 'interact' with the others; i.e. we have

$$
p\left({ }_{a} \mathbb{E}\right)-p\left(\left(E_{\theta_{2,2}}\right)_{\theta_{1,3}}\right)=p\left(\mathbb{E}_{\underline{\theta}_{2,1}}\right)-p(\mathbb{E}) .
$$

Similarly,

$$
p\left(\left(E_{\theta_{2,2}}\right)_{\theta_{1,3}}-p\left(\left(E_{\theta_{2,2}}\right)-\left(p\left(E_{\theta_{1,3}}\right)-p(E)\right)\right.\right.
$$

is a multiple of $\left[B\left(\theta_{2,2}, \theta_{1,3}\right)\right]$.

We note that, by another formula of Mumford [12], we have $\delta_{0} \cdot \delta_{2}=\left(10 \lambda_{1}-2 \delta_{1}\right) \cdot \delta_{2}$. Also, $\delta_{2}^{2}=-\psi_{\Delta_{2}}$ (Faber) and

$$
\delta_{1}^{2}=-\psi_{\Delta_{1}}+2 \delta_{1,1}
$$

by Faber's result together with the fact that $\Delta_{1}$ has normal crossings and multiplicity 2 along the interior of $\Delta_{11}$.

8.2.2. The $(2,1)$ bisep. Here we will compute the unique term of noncompact type: viz. the contribution of the locus $B(\underline{\theta}) \subset \Delta_{0}, \underline{\theta}=\underline{\theta}_{2,1}$ corresponding to curves ${ }_{\mathrm{L}} X,{ }_{\mathrm{R}} X$ of genera 2,1 meeting in 2 points (note the latter specification determines an orientation on $\underline{\theta}$ ). This corresponds to an excessive locus in $X_{B}^{\{2\}}$.

Note that ${ }_{\mathrm{L}} D_{\mathrm{R}} D$ collapses in the unblown-up Hilbert scheme $X_{B}^{[2]}$ while the normal bundles to ${ }_{\mathrm{L}} D,{ }_{\mathrm{R}} D$ are pulled back from there: hence ${ }_{\mathrm{L}} D^{2}{ }_{\mathrm{R}} D={ }_{\mathrm{L}} D_{\mathrm{R}} D^{2}=0$. Also, terms containing Chern classes of $E,{ }_{\mathrm{L}} E,{ }_{\mathrm{R}} E$ cannot contribute to $[B(\underline{\theta})]$. From this it is easy to see that the only term contributing to the fundamental class $[B(\underline{\theta})]$ on $B$ is

$$
-\left[{ }_{\mathrm{R}} D^{2} c_{1}\left(\Lambda_{1}(\omega)\right) \omega_{1}\right]=\underset{50}{-\left(\omega_{1}+\omega_{2}-\Gamma\right) \omega_{1} \mid \mathcal{O}_{\mathrm{R}} D(1)}
$$


( ${ }_{\mathrm{L}} D^{2}$ fails to occur because ${ }_{\mathrm{R}} E^{0}$ has rank 1 ). The latter pushes down to $-2[B(\underline{\theta})]$ as $\omega$ has degree 2 on ${ }_{\mathrm{R}} X$. For the contribution to the hyperelliptic locus in $B$ one must divide by $6=\operatorname{deg}(\omega)$, yielding $(-1 / 3)[B(\underline{\theta})]$. In Faber's notation, $[B(\underline{\theta})]$ is denoted $\gamma_{1}$. Thus,

$$
p\left(\mathbb{E}_{\underline{\theta}}\right)-p(\mathbb{E})=\frac{-1}{6} 2[B(\underline{\theta})]=\frac{-1}{6} 2 \gamma_{1} .
$$

As noted above, because $\partial_{\underline{\theta}}$ has codimension 2 , the same formula holds with $\mathbb{E}$ replaced by its azimuthal modification with respect to any collection of seps.

8.2.3. The $(2,2)$ sep. Here we will use (8.1.12)

To compute $p_{1}\left(\mathbb{E}_{\theta}\right)-p_{1}(\mathbb{E})$ for $\theta=\theta_{2,2}$, note that ${ }_{\mathrm{L}} D \omega_{1}^{2} \omega_{2}=\left({ }_{\mathrm{L}} \omega+{ }_{\mathrm{L}} \theta\right)_{1}^{2}\left({ }_{\mathrm{L}} \omega+{ }_{\mathrm{L}} \theta\right)_{2}$ has base image $\left(2{ }_{\mathrm{L}} g-1\right) i_{\partial_{\theta} *}\left({ }_{\mathrm{L}} \mathcal{K}_{1}+{ }_{\mathrm{L}} \psi\right)$ (here ${ }_{\mathrm{L}} g=2$, but it's best to plug this in only at the end). Ditto ${ }_{\mathrm{L}} D \omega_{1} \omega_{2}^{2}$. Similarly, $\mathrm{L} \omega^{2} \Gamma$ has base image ${ }_{\mathrm{L}} \kappa_{1}+{ }_{\mathrm{L}} \psi$. Therefore in all,

$$
p_{1}\left(\mathbb{E}_{\theta}\right)-p_{1}(E)=(-1 / 6) 6 i_{\partial_{\theta} *}\left({ }_{\mathrm{L}} \mathcal{K}+{ }_{\mathrm{R}} \mathcal{K}+\psi\right) .
$$

For $p_{2}$, note that base classes like $\lambda_{1}$ or $\psi$ on ${ }_{L} D$, when multiplied by $\omega_{1} \omega_{2}-\omega \Gamma$, yield a coefficient of $(2 \mathrm{~L} g-1)(2 \mathrm{~L} g-2)=2\left({ }^{2}{ }_{2}^{g-1}\right)$, which is the fibre degree of $\omega_{1} \omega_{2}-\omega \Gamma$ (here $\omega_{1}^{2}$ has fibre degree 0$)$. On the other hand a term like ${ }_{L} \theta$, which becomes ${ }_{L} \theta_{1}+{ }_{L} \theta_{2}$ on the ordered version, yields ${ }_{\mathrm{L}} \theta_{2}\left({ }_{\mathrm{L}} \omega+{ }_{\mathrm{L}} \theta\right)_{1}^{2}$ when multiplied by $\omega_{1}^{2}$ and zero when multiplied by $\omega_{1} \omega_{2}$ or $\omega \Gamma$ (recall that $\omega \theta=0$ ), therefore it pushes down as above to ${ }_{\mathrm{L}} \mathcal{K}+{ }_{\mathrm{L}} \psi$. So in all we get:

$$
p_{2}\left(\mathbb{E}_{\theta}\right)-p_{2}(\mathbb{E})=(-1 / 6) i_{\partial_{\theta} *}\left(-24 \lambda+18 \psi+2\left({ }_{\mathrm{L}} \mathcal{K}+{ }_{\mathrm{R}} \mathcal{K}+\psi\right)\right) .
$$

Finally, for $p_{3}$, it comes from the part involving ${ }_{\mathrm{L}} \theta^{[2]}$ times a base class, etc., which yields in total

$$
p_{3}\left(\mathbb{E}_{\theta}\right)-p_{3}(\mathbb{E})=(-1 / 6) 6 i_{\partial_{\theta} *}(\lambda) .
$$

All in all, we get

$$
p\left(\mathbb{E}_{\theta}\right)-p(\mathbb{E})=(-1 / 6) i_{\partial_{\theta} *}\left(26 \psi+8\left({ }_{\mathrm{L}} \mathcal{K}+{ }_{\mathrm{R}} \mathcal{K}\right)-30 \lambda\right) .
$$

Using Faber's notation and Mumford's formula in genus two: $\kappa_{1}=2 \lambda_{1}+\delta_{1}$, which applies to ${ }_{\mathrm{L}} \mathcal{K}$ and $\mathrm{R}_{\mathrm{K}} \mathcal{K}$, this can be written as

$$
(-1 / 6)\left(-14 \lambda . \delta_{2}+8 \delta_{2} \delta_{1}-26 \delta_{2}^{2}\right) .
$$

Because $\delta_{0} \cdot \delta_{2}=\left(10 \lambda-2 \delta_{1}\right) \cdot \delta_{2}$, note that this, combined with (8.2.21), already yields $18 / 6,27 / 6$ for the coefficients of $\lambda . \delta_{2}, \delta_{2}^{2}$ in the hyperelliptic class on $B$, in accord with Faber-Pandharipande.

8.2.4. The (1,3) sep. Set $\theta(2)=\theta_{2,2}, E=\mathbb{E}_{\theta_{2}}, \theta:=\theta(1):=\theta_{1,3},{ }_{L} D={ }_{L} D(\theta)$, etc. Here we will compute $p\left(E_{\theta_{1,3}}\right)-p(E)$. We will assume temporarily that the the locus $\partial_{\theta}$ has no double points, i.e. that $\theta$ has at most one point in any fibre. Over $\partial_{\theta_{2,2}, \theta_{1,3}}$ the curve splits as

$$
X=X_{1,1} \cup_{\theta_{1}} X_{1,2} \cup_{\theta_{2}} X_{2}
$$

with components of genera 1,1,2 respectively. Let's denote the corresponding codimension-2 loci in the Hilbert scheme by $D_{1,1} \subset{ }_{\mathrm{L}} D$ and $D_{1,2}, D_{2} \subset{ }_{\mathrm{R}} D$. Note that each of these pushes 
down to $\delta_{2} . \delta_{1}$ and not $\delta_{1,1}$. Using (8.1.12), we can write the restriction of $E$ on the $(1,3)$ boundary as ${ }_{\mathrm{R}} E+{ }_{\mathrm{L}} E$ where, neglecting negligible terms,

$$
\begin{aligned}
& c\left(\left.{ }_{\mathrm{R}} E\right|_{\mathrm{L}} D\right) \sim c\left({ }_{\mathrm{R}} \mathbb{E}\right)+3\left[D_{1,1}\right], \\
& c\left(\left.{ }_{\mathrm{L}} E\right|_{\mathrm{R}} D\right) \sim c\left({ }_{\mathrm{L}} \mathbb{E}\right)+\left[D_{2}\right] .
\end{aligned}
$$

Here $c_{1}\left({ }_{L} \mathbb{E}\right)={ }_{L} \lambda$ is just the pullback of the Hodge class on $\overline{\mathfrak{M}}_{1,1}$. We have used the obvious fact $\theta(2) . D_{1,1}=0$. Now we compute, setting ${ }_{\mathrm{R}} \lambda=c_{1}\left({ }_{\mathrm{R}} \mathbb{E}\right), \psi=\psi\left(\theta_{1,3}\right)$ :

$$
\begin{aligned}
Q\left({ }_{\mathrm{L}} D,{ }_{\mathrm{R}} E\right) & =3-3\left(\psi+\theta_{1}^{[2]}\right)+2{ }_{\mathrm{R}} \lambda+6 D_{1,1}+\theta_{1}^{[2]}\left(2 \psi-{ }_{\mathrm{R}} \lambda-3 D_{1,1}\right), \\
\frac{1}{c\left({ }_{\mathrm{R}} E\right)} & =1-{ }_{\mathrm{R}} \lambda-3 D_{1,1}, \\
{ }_{\mathrm{L}} q:=\frac{Q\left({ }_{\mathrm{L}} D,{ }_{\mathrm{R}} E\right)}{c\left({ }_{\mathrm{R}} E\right)} & =3-3 \psi-3 \theta_{1}-{ }_{\mathrm{R}} \lambda-3 D_{1,1}+\theta_{1}^{[2]}\left(2 \psi-{ }_{\mathrm{L}} \psi+2{ }_{\mathrm{R}} \lambda+6 D_{1,1}\right) .
\end{aligned}
$$

$$
\begin{aligned}
{ }_{\mathrm{R}} E^{0} & \sim{ }_{\mathrm{R}} E-{ }_{\mathrm{R}} \psi-D_{1,1}, \\
Q\left(D,{ }_{\mathrm{R}} E^{0}\left({ }_{\mathrm{L}} D\right)\right) & \sim 2-3\left(\psi+{ }_{\mathrm{L}} \theta_{1}^{[2]}\right)+{ }_{\mathrm{R}} \lambda-{ }_{\mathrm{R}} \psi+3 D_{1,1}, \\
\frac{1}{c\left({ }_{\mathrm{R}} E^{0}\left({ }_{\mathrm{L}} D\right)\right)} & \sim 1-{ }_{\mathrm{R}} \lambda+{ }_{\mathrm{R}} \psi-2 D_{1,1}+\left(\psi+{ }_{\mathrm{L}} \theta_{1}^{[2]}\right)\left(1+{ }_{\mathrm{R}} \lambda-{ }_{\mathrm{R}} \psi+2 D_{1,1}-2 \psi\right), \\
{ }_{\mathrm{L}} q^{0}:=\frac{Q\left(D,{ }_{\mathrm{R}} E^{0}\left({ }_{\mathrm{L}} D\right)\right.}{c\left({ }_{\mathrm{R}} E^{0}{ }_{\mathrm{L}} D\right)} & \sim 2+\left(\psi+{ }_{\mathrm{L}} \theta_{1}^{[2]}\right)-2 D_{1,1}-{ }_{\mathrm{R}} \lambda+{ }_{\mathrm{R}} \psi+\left(\psi+{ }_{\mathrm{L}} \theta_{1}^{[2]}\right)\left(-{ }_{\mathrm{R}} \lambda+{ }_{\mathrm{R}} \psi-2 D_{1,1}\right) .
\end{aligned}
$$

$$
\begin{aligned}
\mathrm{L}_{\mathrm{L}} m\left(\theta_{1}, E\right) & =i_{\mathrm{L} D *}\left({ }_{\mathrm{L}} q+{ }_{\mathrm{L}} q^{0}-\left(\psi+{ }_{\mathrm{L}} \theta_{1}^{[2]}\right)_{\mathrm{L}} q_{\mathrm{L}} q^{0}\right) \\
& =i_{\mathrm{L} D *}\left(5-8\left(\psi+{ }_{\mathrm{L}} \theta_{1}^{[2]}\right)-5 D_{1,1}-2{ }_{\mathrm{R}} \lambda+{ }_{\mathrm{R}} \psi+{ }_{\mathrm{L}} \theta_{1}^{[2]}\left(8 \psi+6{ }_{\mathrm{R}} \lambda+16 D_{1,1}-2{ }_{\mathrm{R}} \psi-4{ }_{\mathrm{L}} \psi\right)\right), \\
\mathrm{R} m\left(\theta_{1}, E\right) & =i_{\mathrm{R} D *}\left(1-{ }_{\mathrm{L}} \lambda-2 D_{2}-2{ }_{\mathrm{R}} \theta_{2}^{[2]} D_{2}\right) .
\end{aligned}
$$

Then

$$
c\left(E_{\theta_{1}}\right)=c(E)\left(1+{ }_{\mathrm{L}} m\left(\theta_{1}, E\right)\right)\left(1+\mathrm{R}_{\mathrm{R}} m\left(\theta_{1}, E\right)\right) .
$$

Now we can compute as in the previous subsection:

$p_{1}\left(E_{\theta}\right)-p_{1}(E)=\frac{-1}{6}\left(5_{\mathrm{L}} D+{ }_{\mathrm{R}} D\right)\left(\omega_{2}^{2} \omega_{1}+\omega_{1}^{2} \omega_{2}-4 \omega^{2} \Gamma\right)=\frac{-1}{6}\left(-10\left({ }_{\mathrm{L}} \mathcal{K}+{ }_{\mathrm{L}} \psi\right)+6\left({ }_{\mathrm{R}} \mathcal{}+{ }_{\mathrm{R}} \psi\right)\right)$.

For $p_{2}$, the portion involving $c_{1}(E) m_{1}$ yields $-\left(24 \delta_{2}+20 \lambda\right) \delta_{1}$; the portion involving $m_{2}$ yields $+8\left({ }_{\mathrm{L}} \mathcal{K}+{ }_{\mathrm{L}} \psi\right)+\left(12 \delta_{2}+20{ }_{\mathrm{L}} \lambda\right) \delta_{1}$ so, considering that ${ }_{\mathrm{L}} \mathcal{K}=0$ and ${ }_{\mathrm{L}} \lambda={ }_{\mathrm{L}} \psi$ in genus 1 , we have in total,

$$
p_{2}\left(E_{\theta}\right)-p_{2}(E)=\frac{-1}{6}\left(-12 \delta_{2}-20 \lambda_{1}+28_{\mathrm{L}} \psi\right) \delta_{1}
$$

For $p_{3}$, the part coming from $c_{2}(E) m_{1}$ yields $-10 \delta_{1} \delta_{2}$ (arising from $-2 \theta(2) \cdot\left({ }_{\mathrm{L}} D(2)+{ }_{\mathrm{R}} D(2)\right)$ ). The part coming from $c_{1}(E) m_{2}$, yields: $-8 \lambda$ from $\lambda\left(-8 \theta(1){ }_{\mathrm{L}} D(1)\right),-24 \delta_{2} \delta_{1}$ arising from $3\left({ }_{\mathrm{L}} D+\right.$ 
$\left.{ }_{\mathrm{R}} D(2)\right) \cdot\left(-8 \theta(1) \cdot{ }_{\mathrm{L}} D(1)\right.$, and finally $18 \delta_{1} \delta_{2}$ arising from $3_{\mathrm{L}} D(2) \cdot 2 D_{2}$; total: $\left(-8 \lambda-6 \delta_{2}\right) \cdot \delta_{1}$. Finally, the part coming from $m_{3}$ yields: $\left(8 \psi+6{ }_{\mathrm{R}} \lambda-2_{\mathrm{R}} \psi+16 \delta_{2}\right) \cdot \delta_{1}$ (arising from multiples of $\theta(1)$. ${ }_{\mathrm{L}} D$ and $-6 \delta_{2} \delta_{1}$ (arising from $-2 D_{2} \theta_{2}$ ). Thus in total:

$$
p_{3}\left(E_{\theta}\right)-p_{3}(E)=\frac{-1}{6} \delta_{1} \cdot\left(-6 \delta_{2}-8 \lambda+8 \psi+6{ }_{\mathrm{R}} \lambda-2_{\mathrm{R}} \psi\right) .
$$

Summing up and using

$$
{ }_{\mathrm{R}} \mathcal{K}=12_{\mathrm{R}} \lambda-{ }_{\mathrm{R}} \delta_{0}-{ }_{\mathrm{R}} \delta_{1}=12 \lambda \cdot \delta_{1}-12_{\mathrm{L}} \lambda-{ }_{\mathrm{R}} \delta_{0}-\delta_{2} \cdot \delta_{1}-\delta_{1,1}=\left(12 \lambda-\delta_{0}-\delta_{2}\right) \cdot \delta_{1}-\delta_{1,1},
$$

we get

$$
p\left(E_{\theta}\right)-p(E)=\frac{-1}{6}\left(4_{\mathrm{L}} \psi+12 \psi+50 \lambda-6 \delta_{0}-24 \delta_{2}-6 \delta_{1,1}\right)_{\delta_{1}}
$$

8.2.5. The $(1,2,1)$ improper bisep. To complete the calculation we must compute the contribution from the locus $\partial_{1,2,1}$ corresponding to the improper bisep of type $(1,2,1)$. This is denoted by $\Delta_{1,1}$ by Faber and equals the double locus of the boundary divisor $\Delta_{1}$. Note that in (8.2.19), rewritten via (8.2.22), the total coefficient of this cycle is $(1 / 6) 30$ where 30 comes from $26=2.13$ from the $\delta_{1}^{2}$ plus 4 . In our azimuthal approach, this locus makes an additional contribution stemming from the fact that the azimuthal bundle is modified there twice, once from each of the seps of type $(1,3)$. To compute this contribution, we may assume by a suitable base change that the global $(1,3)$ sep splits in two components, say $\theta^{\prime}, \theta^{\prime \prime}$ so that the $(1,2,1)$ bisep locus is just $\partial_{\theta^{\prime}, \theta^{\prime \prime}}=\partial_{\theta^{\prime}} \cap \partial_{\theta^{\prime \prime}}$ and the curve over this locus splits as

$$
X_{\theta^{\prime}, \theta^{\prime \prime}}=X^{\prime}(1) \cup_{\theta^{\prime}} X(2) \cup_{\theta^{\prime \prime}} X^{\prime \prime}(1)
$$

(respective genera 1,2,1). Denote the corredponding Hilbert loci of schemes contained in the respective curve by $D^{\prime}(1), D(2), D^{\prime \prime}(1)$. We then calculate the $\partial_{1,2,1}$ term in

$$
\left(1+{ }_{\mathrm{L}} m\left(\theta^{\prime}, E\right)+{ }_{\mathrm{R}} m\left(\theta^{\prime}, E\right)\right)\left(1+{ }_{\mathrm{L}} m\left(\theta^{\prime \prime}, E_{\theta^{\prime}}\right)+{ }_{\mathrm{R}} m\left(\theta^{\prime \prime}, E_{\theta^{\prime}}\right)\right) .
$$

The first factor is analogous to the above for $\theta_{1}$; the second is likewise analogous, except for the additional term of $+6 \theta^{\prime \prime} . D(1)$. Then, neglecting terms that do not affect the coefficient of $\delta_{1,1}$, the above product becomes

$$
\begin{aligned}
& \left(1+5_{\mathrm{L}} D\left(\theta^{\prime}\right)-8 \theta^{\prime}{ }_{\mathrm{L}} D\left(\theta^{\prime}\right)+{ }_{\mathrm{R}} D\left(\theta^{\prime}\right)\right) \cdot\left(1+5_{\mathrm{L}} D\left(\theta^{\prime \prime}\right)-8 \theta^{\prime \prime}{ }_{\mathrm{L}} D\left(\theta^{\prime \prime}\right)+{ }_{\mathrm{R}} D\left(\theta^{\prime \prime}\right)\right) \sim \\
& -8 \theta^{\prime} \cdot D^{\prime}(1)-2 \theta^{\prime \prime} D^{\prime \prime}(1)+D(2) .
\end{aligned}
$$

This contributes a term of

$$
(-1 / 6)(-22) \partial_{1,1}
$$

to $p\left({ }_{a} E\right)$. Adding this to $(-1 / 6)\left(-6 \delta_{1,1}\right)$ from (8.2.37) and $(-1 / 6)(-2) \delta_{1,1}$ from (8.2.21), we get a total $\delta_{1,1}$ term of $(-1 / 6)(-30) \delta_{1,1}=(1 / 6)(13.2+4) \delta_{1,1}$ matching (8.2.19).

8.2.6. Finale. Now adding together (8.2.21), (8.2.23), (8.2.28), (8.2.37) and (8.2.40) we recover (8.2.19). 
8.3. W-bundles. As discussed in $\$ 5.2$ the exceptional divisors in an $\mathcal{S}$-stratified blowup have a $W_{n}$-bundle structure and the quantitative, enumerative aspect of this structure is involved in computing azimuthal modifications corresponding to biseps (see (8.1.17)). Our purpose here is to study $W_{n}$-bundle structures and their intersection theory generally.

Let $\mathcal{L}=\left(L_{0}, \ldots, L_{n}\right)$ be a collection of line bundles on a variety $Z$, and set, for $I \subset\{0, \ldots, n\},|I|>$ 1 ,

$$
\begin{array}{r}
L_{+}:=\bigoplus_{i=0}^{n} L_{i} \supset L_{I}=\bigoplus_{i \in I} L_{i}, \\
P_{+}=\mathbb{P}\left(L_{+}\right), P_{I}=\mathbb{P}\left(L_{I}^{*}\right), P=P_{+} \times \prod_{2 \leq|I| \leq n} P_{I}
\end{array}
$$

(all products relative over $Z$ ) and let $Q_{I}$ be the tautological quotient bundle of rank $|I|-1$ of $L_{I}^{*}$, i.e. the dual of the tautological subbundle on $P_{I}$ (pulled back to $P$ ). Then on $P$, we have natural composite maps

which together induce

$$
\mathcal{O}_{P_{+}}(-1) \rightarrow L_{+}^{*} \rightarrow L_{I}^{*} \rightarrow Q_{I}
$$

$$
\mathcal{O}_{P_{+}}(-1) \rightarrow \bigoplus_{2 \leq|I| \leq n} Q_{I}
$$

The zero locus of the latter map will be temporarily denoted by $W^{\prime}$. It consists of collections $\left(h ;\left(h_{I}\right)\right)$ where $h$ is a hyperplane in $L_{+}$and $h_{I}$ is a hyperplane in $L_{I}$ contained in $h$. The image of $W^{\prime}$ in $\prod_{i<j} P_{i, j}$ is the locus denoted earlier (see $\$ 5.2$, (5.2.1) ) by $W[\mathcal{L}]=W\left[L_{0}, \ldots, L_{n}\right]$. Note that the closure of the graph of a linear projection $P_{+} \rightarrow P_{I}$ can be identified with the blowup of $\mathbb{P}\left(L / L_{I}\right) \subset P_{+}$. Applying this to the components of the projection of $W^{\prime}$ to $\prod_{|I|=2} P_{I}$, it follows that the image of the projection can be identified with $W[\mathcal{L}]$, i.e. the normal blowup, hence by Proposition 5.2, also with the $S$-stratified blowup of $P_{+}$corresponding to the stratification by coordinate planes, which is smooth.

Lemma 8.1. (i) $W^{\prime}$ projects isomorphically to its image $W[\mathcal{L}] \subset \prod_{|I|=2} P_{I}$;

(ii) this image is equal to the degeneracy $($ rank $\leq n)$ locus of the natural map

$$
\bigoplus_{|I|=2} Q_{I}^{*} \rightarrow L_{+}
$$

and this map has rank at least $n$ everywhere.

Proof. By smoothness of $W[\mathcal{L}]$, it suffices to prove (ii) plus the bijectiveness part of (i). This in turn is a consequence of following elementary fact: given a collection of points $A=\left(A_{i, j} \in P_{i, j}\right)$, one on each line of the 1-skeleton of the coordinate simplex in $\mathbb{P}^{n}$, they span at least a hyperplane. The proof is by induction on $n$, the case $n=2$ being obvious. Assuming the case for $n$, consider the case of $n+1$. If those among the $A_{i, j}$ that lie on $\mathbb{P}^{n}$, i.e. those with $i, j \leq n$, span $\mathbb{P}^{n}$, there is nothing to prove. Else, by induction those points lie on a unique hyperplane $H \subset \mathbb{P}^{n}$. Then there exists $k$ such that the $k$-th coordinate point $e_{k} \in \mathbb{P}^{n} \backslash H$. But then $P_{k, n+1} \cap H=\varnothing$ so $A_{k, n+1} \notin H$, therefore $A$ spans at least a hyperplane in $\mathbb{P}^{n+1}$. 
Now set $q_{+}=c_{1}\left(\mathcal{O}_{P_{+}(1)}\right), q_{I}=c_{1}\left(\mathcal{O}_{P_{I}}(1)\right)$. Then because $W^{\prime}$ is the zero locus of the map (8.3.1), the fundamental class of $W^{\prime}$ on $P$ is

$$
\left[W^{\prime}\right]=\prod_{2 \leq|I| \leq n} c_{|I|-1}\left(Q_{I}\left(q_{+}\right)\right)=\prod_{\substack{I \leq|I| \leq n \\ I}}\left[\frac{\prod_{i \in I}\left(1-\left[L_{i}\right]+q_{+}\right)}{1-q_{I}+q_{+}}\right]_{|I|-1} .
$$

This formula easily allows us to compute intersections of standard classes on $W$, as they are pulled back via $W^{\prime} \hookrightarrow P$ :

Proposition 8.2. We have

$$
\pi_{(W[\mathcal{L}] \rightarrow Z) *}\left(q_{+}^{m} \prod q_{I}^{m_{I}}\right)=\pi_{(P \rightarrow Z) *}\left(q_{+}^{m} \prod_{2 \leq|I| \leq n} q_{I}^{m_{I}}\left[\frac{\prod_{i \in I}\left(1-\left[L_{i}\right]+q_{+}\right)}{1-q_{I}+q_{+}}\right]_{|I|-1}\right) .
$$

Remark 8.3. The argument of $\pi_{(P \rightarrow Z) *}$ in (8.3.3) is a polynomial in $q_{+}$and the $q_{I}$. Note that (where s. denotes Segre class)

$$
\begin{array}{r}
\pi_{(P \rightarrow Z) *}\left(q_{+}^{m} \prod_{I} q_{I}^{m_{I}}\right)=s_{m-n}\left(L_{+}\right) \prod_{I} s_{m_{I}-|I|+1}\left(L_{I}\right) \\
=\left[\prod_{i=0}^{n} \frac{1}{1+\left[L_{i}\right]}\right]_{m-n} \prod_{I}\left[\prod_{i \in I} \frac{1}{1+\left[L_{i}\right]}\right]_{m_{I}-|I|+1} .
\end{array}
$$

Consequently, the LHS of (8.3.3) can be computed as a polynomial in the $c_{1}\left(L_{i}\right)$.

By Theorem 5.6 and Remark 5.7, the classes appearing in (8.3.3) are precisely what is needed to compute powers of the exceptional divisors on the azimuthal Hilbert scheme. Indeed in the notation of that Theorem, we have, identifying $\Theta$ with $\{0, \ldots, n\}$ :

$$
c_{1}\left(L_{\underline{\theta}}(\Theta)\right)=q_{+}, c_{1}\left(L_{\underline{\theta}}\left(\Theta, \Theta^{\prime}\right)\right)=q_{\Theta^{\prime}} .
$$

The self-intersection of $\Xi_{\underline{\theta}}(\Theta)$ can be computed using (5.4.7) and Remark 5.7.

\section{REFERENCES}

1. T. Bleier, Excess Porteous, coherent Porteous, and the hyperelliptic locus in $\bar{M}_{3}$, Mich. math. J. 61 (2012).

2. D. Eisenbud, Commutative algebra with a view towards algebraic geometry, GTM 150, Springer, 1995.

3. E. Esteves, The stable hyperelliptic locus in genus 3: an application of Porteous formula, arxiv.org/1310.5561.

4. C. Faber, Chow rings of moduli spaces of curves II : some results on the Chow ring of $M_{4}$, Ann. math. 132 (1990), $421-449$.

5. __ Algorithms for computing intersection numbers on moduli spaces of curves etc., New trends in Algebraic Geometry, Cambridge, 1999, pp. 93-109.

6. C. Faber and R. Pandharipande, Relative maps and tautological classes, J. Euro. Math. Soc. 7 (2005), 13-49.

7. W. Fulton, Intersection theory, Ergeb. d. Math. u. i. Grenzgeb. 3. Folge, Bd. 2, Springer, Berlin, 1984.

8. M. Golubitsky and V. Guillemin, Stable mappings and their singularities, GTM 14, Springer, 1973.

9. J. Harris and D. Mumford, On the Kodaira dimension of the moduli space of curves, Invent. math. (1982), $23-86$.

10. J.Harris and L. Tu, Chern numbers of kernel and cokernel bundles, Inventiones math. (1984), 467-475.

11. R.K. Lazarsfeld, Positivity in algebraic geometry, Springer, 2004.

12. D. Mumford, Towards an enumerative geometry of the moduli space of curves, Arithmetic and Geometry, part II (M. Artin and J. Tate, eds.), Birkhauser, Boston, 1983, pp. 335-368.

13. Z. Ran, Structure of the cycle map for Hilbert schemes of families of nodal curves, 1-34, http://arXiv.org/0903.3693

14. Curvilinear enumerative geometry, Acta Math 155 (1985), 81-101.

15. __ Geometry on nodal curves, Compositio math 141 (2005), 1191-1212. 
16. Echelon modifications of vector bundles, Comm. Alg. 41 (2013), 1846-1853.

17. _ Tautological module and intersection theory on Hilbert schemes of nodal curves, Asian J. Math. 17 (2013), 193264, arxiv:0905.2229v5

18. Canonical systems and their limits on stable curves, J. Algebra 399 (2014), 634-656, arXiv.org/math.AG/1104.4747

19. R. Vakil, The moduli space of curves and Gromov-Witten theory, Enumerative invariants in algebraic geometry and string theory (K. Behrend and M. Manetti, eds.), Springer-Verlag, 2008, arxiv.org/math. AG/0602347

ZIV RAN

UNIVERSITY OF CALIFORNIA

Mathematics Department, Big Springs RD. Surge Facility

RIVERSIDE CA 92521 US

ZIV.RAN @ UCR.EDU 